American Journal of Applied Sciences 7 (7): 987-1022, 2010

ISSN 1546-9239

(C) 2010 Science Publications

\title{
A New Novel Fidelity Digital Watermarking Based on Adaptively Pixel- Most-Significant- Bit-6 in Spatial Domain Gray Scale Images and Robust
}

\author{
${ }^{1}$ Mehemed Bashir Aliwa, ${ }^{1}$ Tarek El-Ahmady El-Tobely, ${ }^{1}$ Mahmood M. Fahmy, \\ ${ }^{2}$ Mohamed EL Said Nasr and ${ }^{3}$ Mohamed Hashem Abd El-Aziz \\ ${ }^{1}$ Department of Computer and Control Engineering, \\ ${ }^{2}$ Department of Electronics and Electrical Communications, \\ Faculty of Engineering, Tanta University, Egypt \\ ${ }^{3}$ Department Information Systems, Faculty of Computers and Information Science, \\ Ain Shams University, Egypt
}

\begin{abstract}
The digital watermarking technology is a way to apply digital information hiding techniques and prevent malicious and non-malicious attacks to detect hidden information. Problem statement: The problems in digital watermarking is that the three requirements of imperceptibility, capacity and robustness that are must be satisfied but they almost conflict with each other, accordingly there are tradeoff between fidelity and robustness. Furthermore the embed a watermark bits within the pixels by a Least-Significant-Bit (LSB) insertion of the cover image in spatial domain technologies, when an image is being embedded, it shouldn't cause any visual change to the cover image, while almost authors using LSB insertion to hide a watermark bits within a low embedding errors, whereas the authors are avoiding to use the Most-Significant-Bit (MSB). Thus there are a trade-off between the embedding error in the LSB and MSB. Approach: We proposed a new novel fidelity and robust of watermark embedding method that satisfies the requirements and problems, called Adaptively Pixel Adjustment Process based on Medial Pyramid Of Embedding Error applying in the Falling-Off-Boundary in Corners Board of the cover image set-of-the Most-Significant-Bit-6 in spatial domain (APAP-MPOEE-FOBCBмSB6). In addition, the study provides a comprehensive overview and analysis of previous methods. Results: Theoretically analysis of the proposed technique proves the effectiveness of the technique in the average of worst case and minimizing the number of embedding error to the half and the experimental results applied on the different benchmark of six gray scale images with two quantum of watermark bit embedded are compared with previous researches and was found better. Moreover in all different benchmark of test images the watermarks were extracted from watermark degrading, removal and geometric transformations attacks to an acceptable degree of similarity function and normalized cross correlation. Conclusion: Theoretically analysis is proves and in all different benchmark the watermarks are extracted under malicious and nonmalicious attacks and compared with previous study was found better.
\end{abstract}

Key words: Fidelity, digital watermarking, imperceptible, spatial domain, LSB and MSB, benchmark

\section{INTRODUCTION}

Digital watermarking is a technique which allows an individual to add hidden copyright notices or other verification messages to digital audio, video, or image signals and documents. Such a message is a group of bits describing information pertaining to the signal or to the author of the signal (name, place). The technique takes its name from watermarking of study or money as a security measure (Cox et al., 2001). Digital watermarking can be a form of steganography (Yusnita and Khalifa, 2007; Lu, 2005) in which data is hidden in the message without the end user's knowledge. In the term of hiding can refer to either for information imperceptibility (watermarking) or information secrecy (steganography) means that the existence of a message is secret, thus the steganography is the art of concealed communication (Yusnita and Khalifa, 2007; Wang, 2006; Cox et al., 2001). Digital watermarking refers to techniques that are used to protect digital data by imperceptibly embedding watermark into the original data in such a way that always remains present (Neil and Jajodia, 1998; Mauro and Bartolini, 2004). Watermarking and steganography are two important

Corresponding Author: Mehemed Bashir Aliwa, Department of Computer and Control Engineering, Faculty of Engineering, Tanta University, Egypt 
sub disciplines of information hiding that are closely related to each other and may be coincide but with different underlying properties, requirements and designs, thus result in different technical solutions (Yusnita and Khalifa, 2007; Cox et al., 2001). Moreover the digital watermarking differs from Cryptography, where cryptography is the art of sending a message by converting it into a secret code called as cipher text. The conversion is done using an algorithm and a secret key. Once the receiver receives the cipher text, he can decode it and convert it into plaintext using his private key. Here, the very existence of the message is not being kept secret but only the contents are. This rouses suspicion and curiosity. On the other hand, the digital watermarking, unlike cryptography, leaves the original medium or data almost unaltered even after embedding it with the copyright information. The naked eye cannot tell the difference in the alteration. The main purpose of using watermarks is to convey ownership, protect copyrighted materials from being illegally distributed and to prevent various other kinds of fraud. In certain instances it is also used in security applications like the ID cards or covert communication (Defense and Intelligence applications). On the other hand in digital watermarking has the additional concept of resilience against attempts to remove the hidden data. This is because the information hidden by watermarking systems is always associated to the digital object to be protected its owner, while steganographic systems just hide any information. Robustness criteria are also different since steganography mainly concerns with detection of hidden message while watermarking concerns potential removal by a pirate. Besides, steganography typically relates to covert point-to-point communication while watermarking is usually one-to-many (Yusnita and Khalifa, 2007; Katzenbeisser and Petitcolas, 1999).

History: Although study was invented in China over a thousand years ago, the Europeans only began to manufacture it in the 11th and 12th centuries, after Muslims had established the first study mills in Spain. Soon after its invention, Chinese merchants and missionaries transmitted paper and knowledge of papermaking, to neighboring lands such as Japan, Korea and Central Asia. It was there that Muslims first encountered it in the 8th century. Islamic civilization spread knowledge of study and papermaking to Iraq, Syria, Egypt, North Africa and finally, Spain. Most accounts of the history of study focus either on its origins in China or its development in Europe. This explains why the oldest watermarked paper found in archives dates back to 1292, in Fabriano, Italy (Katzenbeisser and Petitcolas, 1999). The marks were made by adding thin wire patterns to the study molds. The study would be slightly thinner where the wire was and hence more transparent. At the end of 13th century about 40 paper mills were sharing the study market in Fabriano and producing paper with different format, quality and price (Yusnita and Khalifa, 2007). The digitization of today's world has expanded the watermarking concept to include digital approaches for use in authenticating ownership claims and protecting proprietary interests. Digital Watermarking became famous only in the early of 1990 the idea of digital watermarking, embedding imperceptible information using digital images (Arnold et al., 2003). This was due to the growth of the Internet. The Internet was a big factor in propelling the growth because illegal distribution of copyrighted material became very easy. File sharing technology grew and companies made it easy for users to share for example music and other copyrighted materials like video. This cost the entertainment industry in the millions if not in billions of dollars of lost revenue. This was one of the primary reasons for the rapid development of digital watermarking. The first publication in 1993, when Tirkel et al. (1993) presented technique to hide data in image. The method based on modification to the Least Significant Bit (LSB) of the pixel values (Lu, 2005). Since then worldwide research activities have been increasing dramatically and the industrial interest in digital watermarking methods keeps growing.

General framework for watermarking: In general, any watermarking scheme consists of three parts. The watermark, encoder (insertion algorithm) and decoder with comparator (verification or extraction or detection algorithm). All watermarking methods share the same generic building blocks a watermark embedding system also called (Encoder process or insertion algorithm) and a watermark recovery system (also called watermark extraction or watermark decoder) shown in Fig. 1 (Martin and Petitcolas, 2000; Yusnita and Khalifa, 2007; Muhammad and Dot, 2003; Katzenbeisser and Petitcolas, 1999; Lu, 2005).

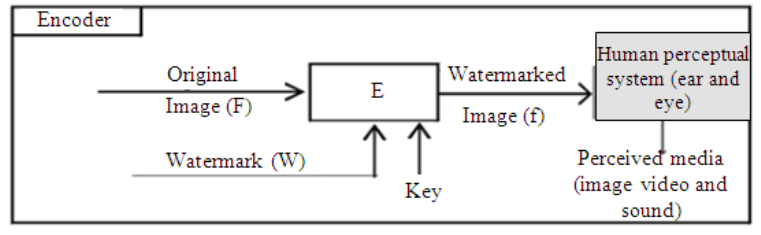

Fig. 1: Encoder process of watermarking 
Am. J. Applied Sci., 7 (7): 987-1022, 2010

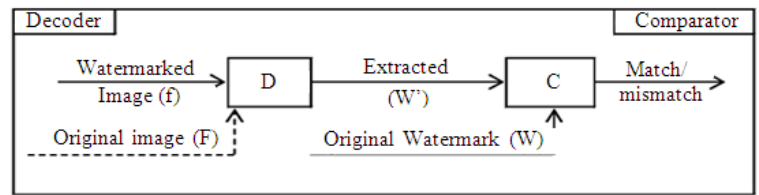

Fig. 2: Decoder process of watermarking

Encoding process: The input to the scheme is the watermark, the cover-original image $\mathrm{F}$ and an optional public or secret key. The watermark can be of any nature such as a number, text, or an image. The key used to enforce security that is the prevention of unauthorized parties from recovering and manipulating the watermark. All practical systems employ at least one key, or even a combination of several keys. In combination with a secret or a public key the watermarking techniques are usually referred to as secret and public watermarking techniques, respectively. The output of the watermarking scheme is the watermarked image $f_{(x, y)}$. Mathematically:

$$
E\left[F_{(x, y)}, k \times W_{(x, y)}\right]=f_{(x, y)}
$$

Where:

$\mathrm{F}_{(\mathrm{x}, \mathrm{y})}=$ Denotes the actual cover image

$\mathrm{W}_{(\mathrm{x}, \mathrm{y})}=$ Denotes the watermark image

$\mathrm{K}=$ Denotes the public or secret key

$\mathrm{f}_{(\mathrm{x}, \mathrm{y})}=$ Denotes the watermarked image

From the above equation, if the watermark insertion process is designed correctly, the result is media that appears identical to the original when perceived by a human, but which yields the encoded watermark information when processed by a watermark detector.

Decoding process: The decoding process is depicted in Fig. 2. Inputs to the scheme are the watermarked data, the secret or public key and, depending on the method, the original data and/or the original watermark. The output is either the recovered watermark W. Three types of watermarking systems can be identified. Their difference is in the nature and combination of inputs and outputs. A decoder function D takes an image (can be a watermarked or un-watermarked image and possibly corrupted) whose ownership is to be determined and recovers a watermark W' from the image. In this process an additional image $F_{(x, y)}$ can also be included which is often the original and unwatermarked version of $f_{(x, y)}$, this information can be referred to as a "key" that is the level of availability of the key in turn determines who is able to read the watermark. Mathematically:

$$
\mathrm{D}\left[\mathrm{f}_{(\mathrm{x}, \mathrm{y})}, \mathrm{F}_{(\mathrm{x}, \mathrm{y})}\right]=\mathrm{W}_{(\mathrm{x}, \mathrm{y})}
$$

A watermark must be detectable or extractable $\mathrm{W}(\mathrm{x}, \mathrm{y})$ to be useful. Depending on the way the watermark is inserted and depending on the nature of the watermarking algorithm.

Comparison process: The comparison process is depicted in Fig. 2, the extracted payload $\mathrm{W}_{(\mathrm{x}, \mathrm{y})}^{\prime}$ is compared with the original payload $\mathrm{W}_{(\mathrm{x}, \mathrm{y})}$ (i.e., the payload that was initially embedded) by a comparator function and a binary output decision is generated. The comparator is basically a correlator depending on the comparator output it can be determined if the data is authentic or not, for e.g., Using a Normalized Cross Correlation (NCC) or Similarity function (SM), whereas the similarity values NCC and SM of about 0.75 or above is considered acceptable (Ali, 2007; Kamran et al., 2006; Muhammad and Dot, 2003).

Types of digital watermarking: Watermarks and watermarking techniques can be divided into various categories in various ways. Watermarking techniques can be divided into five categories according to the type of document to be watermarked as follows: image, video, text and audio watermarking (Lu, 2005). In other way, the digital watermarks can be divided into four different types according to human perception as follows, visible watermark, invisible robust watermark, invisible fragile watermark and Dual watermark. Visible watermarking: The idea behind the visible watermark is very simple; a visible watermark makes slight modifications to an image. The transformation is such that the image can still be seen, but the watermark is effectively laid over the top of it. One of the advantages of visible watermarks is that even if an image is printed and scanned the watermark is still visible (Kevin et al., 2005). It is equivalent to stamping a watermark on study and for this reason is sometimes said to be digitally stamped. An example of visible watermarking is provided by television channels, like $\mathrm{BBC}$, whose logo is visibly superimposed on the corner of the TV picture (Lu, 2005). Invisible watermarking: a pattern is applied to a file or image so that it is undetectable by the human eye. With an invisible watermark you can change certain pixels in an image so his human eye cannot tell the difference from the original image the strength of invisible watermarks is that the image quality is not degraded or changed according to the user or consumer. Invisible watermarks are effective, though, only while the image is in digital form. If a digital image that has an invisible watermark is printed out and then rescanned, the watermark is 
effectively removed (Kevin et al., 2005). On the other hand, it is a far more complex concept? It is most often used to identify copyright data, like author, distributor and so forth (Lu, 2005). Invisible fragile watermarking: fragile are embedded with very low robustness (Arnold et al., 2003). Invisible fragile watermarks are ready to be destroyed by random image processing methods. The change in watermark is easy to be detected (Yusnita and Khalifa, 2007). The fragile watermarks are used to detect any corruption of an image. In some application, we want exactly the opposite of robust (Muhammad and Dot, 2003). The main application of fragile watermarking is data authentication, where watermark loss or alteration is taken as evidence that data has been tampered with (Mauro and Bartolini, 2004). Semi-fragile Watermarking: the idea is to insert a watermark in the original image in such a way that the protected image can undergo some specific image processing operations while it is still possible to detect malevolent alterations and to locate and restore image regions that have been altered (Sanghyun et al., 2002; Adil and Noumeir, 2008). Furthermore watermarks can help localize the exact location where the tampering of the cover study occurred ( $\mathrm{Lu}, 2005)$. Watermark is semi-fragile if it survives a limited well specified, set of manipulations, leaving the quality of the host document virtually intact. Dual watermarking: is a combination of a visible and an invisible watermark. In this type of watermark an invisible watermark is used as a backup for the visible watermark as clear from the following diagram (Mohanty et al., 1999) as shown in Fig. 3.

From application point of view digital watermark could be source based or destination based (Yusnita and Khalifa, 2007). Source-based watermark are desirable for ownership identification or authentication where a unique watermark identifying the owner is introduced to all the copies of a particular image being distributed. A source-based watermark could be used for authentication and to determine whether a received image or other electronic data has been tampered with. The watermark could also be destination-based where each distributed copy gets a unique watermark identifying the particular buyer. The destination-based watermark could be used to trace the buyer in the case of illegal reselling.

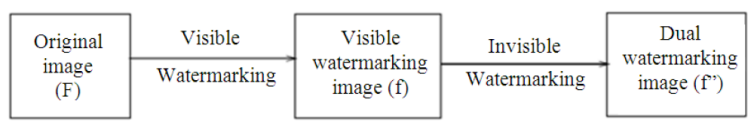

Fig. 3: Schematic representation of dual watermarking
Characteristics of a watermarking: There are a number of important characteristics that watermarks exhibit. Imperceptibility: means that the perceived quality of the host image should not be distorted by the presence of the watermark (Ali, 2007). Imperceptibility due to the particular nature of the authentication task, it is usually necessary that watermark imperceptibility is guaranteed. Nevertheless, some applications may exist in which a slightly perceptible watermark is allowed (Mauro and Bartolini, 2004). Furthermore the imperceptibility for hidden information (Bijay et al., 2005). Moreover the modifications caused by watermark embedding should be below the perceptible threshold, which means that some sort of perceptibility criterion should be used not only to design the watermark, but also quantify the distortion. As a consequence of the required imperceptibility used for watermark embedding are only modified by a small amount (Katzenbeisser and Petitcolas, 1999). Fidelity: This refers to the term imperceptible as it is referred in the literature of watermarks (Yusnita and Khalifa, 2007; Mehemed et al., 2009; Bansall and Bhadouria, 2007). The watermark should not be noticeable to the viewer nor should the watermark degrade the quality of the content (Cox et al., 2001; Muhammad and Dot, 2003). The fidelity of a watermarked signal depends on the amount of embedded information, the strength of the mark and the characteristics of the host signal (Lu, 2005). Perceptual Transparency: Refers to the property of the watermark of being imperceptible in the sense that humans can not able distinguish the watermarked images from the original ones by simple inspection (Kevin et al., 2005; Franco and Gomez, 2008), where the embed data without affecting the perceptual quality of the host signal (Fabien, 2000). Undetectability: The aim of the undetectability as well as the removal attacks is to render the embedded watermark undetectable (Arnold et al., 2003). Additionally, we say that a watermark is Wide-Sense Reversible (WSR) if once it has been decoded/detected it can be made undecodable/undetectable without producing any perceptible distortion of the host asset (Mauro and Bartolini, 2004). It should not be possible for an attacker to find any significant statistical differences between an unmarked signal and a marked signal (Fabien, 2000). Data payload: refers to the amount of information stored in the watermark, which in general depends on the application (Franco and Gomez, 2008; Bansall and Bhadouria, 2007). For a photograph, the data payload would refer to the number of bits encoded within the image. For audio, data payload refers to the number of embedded bits per second that are transmitted. For video, the data payload may refer to 
either the number of bits per field (or frame) or the number of bits per second (Cox et al., 2001). Capacity: knowing how much information can reliably be hidden in the signal is very important to users especially when the scheme gives them the ability to change this amount. Moreover refers to the bit size of a payload that a watermark access unit can carry (Fabien, 2000). How many marks can be added simultaneously (Matt et al., 1999)? Security: The security of a watermark refers to its ability to resist hostile attacks (Cox et al., 2001). The embedded watermark cannot be removed beyond reliable detection by targeted attacks based on a full knowledge of the embedding algorithm and the detector (except a secret key). Computational cost: The time that it takes for a watermark to be embedded and detected can be a crucial factor in a watermarking system (Lu, 2005). On the other hand speed be sides fidelity, where the content owner might be interested in the time it takes for an algorithm to embed a mark. Although speed is dependent on the type of implementation (hardware or software), some applications require real time embedding and/or detection (Fabien, 2000; Lu, 2005). Moreover the efficiency of computing time in storage requirements and software or hardware size of the mark writing and reading processes? Are they realtime, so that they can be incorporated into playback or display mechanisms in an on-line setting (Matt et al., 1999). Data secrecy (secret keys): What information needs to be retained, or kept secret, about the marks, their meaning and the marked material? Depending upon the watermarking method, such information can include encryption and decryption keys for computing and interpreting marks (Matt et al., 1999). A watermark should usually be secret and only accessible by authorized parties. Knowledge of a watermark inserter or detector can make a method more vulnerable to attack. For more protection to the watermark bits a secret-Key has been used to permute the watermark bits before embedding it to achieve cryptographic security (Aiad and Sada, 2007). In general, watermarking systems should use one or more cryptographically secure keys (called watermark keys) to ensure that the watermark cannot be manipulated ( $\mathrm{Lu}, 2005)$. Robustness: The ability of the watermark to survive normal processing of content (Cox et al., 2001). Moreover refers to the capacity of the watermark to remain detectable after alterations due to processing techniques or intentional attacks (Franco and Gomez, 2008). The watermark should be resistant to distortion introduced during either normal use (unintentional attack), or a deliberate attempt to disable or remove the watermark present (intentional, or malicious attack). Unintentional attacks involve transforms that are commonly applied to images during normal use, such as for example cropping, noise, scaling and compression (Bijay et al., 2005). Accuracy of detection: How accurately can the mark be read? What is the chance of a false positive (unmarked content appearing to have a mark) (Fabien, 2000), a false negative (marked data appearing to be unmarked), or a false reading (a mark misread as another mark) (Matt et al., 1999). Redundancy: To ensure robustness, the watermark information is embedded in multiple places on the cover data file. This means that the watermark can usually be recovered from just a small portion of the watermarked file ( $\mathrm{Lu}, 2005)$. The watermark information is usually redundantly distributed over many samples like pixels or features of the cover data (Katzenbeisser and Petitcolas, 1999). Furthermore redundancy in distribution of the hidden information inside the cover image to satisfy robustness in watermark extraction process even from the truncated (cropped) watermarked image (Bijay et al., 2005). The redundancy of the data helps to hide the existence of a secret message (Kevin et al., 2005).

Watermarking system: Private watermarking systems (or called non-blind watermarking) (Katzenbeisser and Petitcolas, 1999; Eugene, 2007) require at least the original data in the reading process (Arnold et al., 2003). It guarantees better robustness but may lead to multiple claims of ownerships (Lin, 2005). Public watermarking systems (or called blind or oblivious watermarking) (Katzenbeisser and Petitcolas, 1999; Eugene, 2007) means watermark detection and extraction do not depend on the availability of original image. It is the biggest challenge to the development of a watermarking system (Schyndel et al., 1994). The drawback is when the watermarked image is seriously destroyed; watermark detection will become very difficult (Lin, 2005). Semi-blind watermarking systems (or called semiprivateor semi blind watermarking) (Katzenbeisser and Petitcolas, 1999; Eugene, 2007), as a subclass of blind system (Lin, 2005), is capable of detecting only the presence of the embedded symbol with the help of secret key and the watermark symbol but without the cover image (Bijay et al., 2005).

In recent years, watermarking has become an attractive topic and many watermarking schemes have been proposed. The current watermarking techniques can be grouped into categories (Eugene, 2007) in spatial domain (Kevin et al., 2005; Chan and Cheng, 2004; Aiad and Sada, 2007; Yang, 2008; Santi and Kundu, 2002; Neil and Jajodia, 1998; Schyndel et al., 1994; Tirkel et al., 1993), in frequency domain (Ali, 2007; Franco and Gomez, 2008; Yusnita and Khalifa, 
2007; 2008; Sanghyun et al., 2002; Kamran et al., 2006; Muhammad and Dot, 2003) and feature domain (Eugene, 2007). Among these schemes, the ones which require the original information and secret keys for the watermarking extraction are called private watermark schemes (Yusnita and Khalifa, 2008; Sanghyun et al., 2002; Kamran et al., 2006; Muhammad and Dot, 2003). Schemes which require the watermark information and secret keys are called semi-private or semi-blind schemes. Schemes which need secret keys rather than the original information are called public or blind watermark schemes (Aiad and Sada, 2007; Ali, 2007; Mehemed et al., 2009; Santi and Kundu, 2002).

The study is organized as follows, first: describes the problem definition. Second: describes the principle of previous study. Third: describes the performance evaluation of watermarking system. Four: describes the study with analysis and modified previous study. Six: describes the proposed method insertion, extraction and analysis of watermarking scheme. Seven: experimental of performance results and discussion computed in two parts (i): Theoretically analysis and (ii): Applied on the different benchmark of six gray scale images and two quantum of watermark bit embedded are compared with an previous study and modified algorithms. Finally, conclusion and future study.

Problem definition: The digital watermarking technology is a way to apply digital information hiding techniques, including the ability to hide digital information inside digital images (gray scale images), to prevent malicious and non-malicious attacks to detect hidden information. The problem in digital watermarking is that there are three requirements of imperceptibility, capacity and robustness which must be satisfied but they almost always conflict with each other, in the same case there are trade-off between fidelity and robustness. Accordingly, the proposed solution is to embed a watermark image within the pixels of the cover image in spatial domain technologies, but still there is another problem, (i): when an image is being embedded, it shouldn't cause any visual change to the cover image, whereas almost authors using a Least-Significant-Bit (LSB) insertion in spatial domain to hide a watermark image (Chan and Cheng, 2004; Aiad and Sada, 2007; Yang, 2008; Santi and Kundu, 2002; Lesley et al., 1998; Neil and Jajodia, 1998; Schyndel et al., 1994) or (massage) within a low embedding errors, where the authors are avoiding to use the Most-Significant-Bit (MSB). While the statement problem here, there are a trade-off between the embedding error in the LSB and MSB. Furthermore the embedding process in the LSB do not introduce any perceptible into the cover image, as well as the embedding errors in the LSB growth up from (1- $\left.8_{\text {Max }}\right)$. While in the MSB growth up from (16-128 $8_{\text {Max }}$, with introducing high perceptible into the cover image. On the other hand the authors investigated into the use of the LSB substitution technique in digital watermarking (Kevin et al., 2005) is described in previous methods, the authors say that the LSB embedded watermark bits can easily be removed using techniques that do not affect the image visually to the point of being noticeable and if the watermark is hidden in the LSB, all the individual has to do is flip one LSB, thus the information cannot be recovered, by the way in recent years the techniques in spatial domain technologies they are becoming generally abandoned. (ii): Another problem appears with this since the image is limited by its dimensions, the number of bits that are usable for embedding is also limited and the watermark image should be chosen in such that it could fit in the cover image. From these problems we aim at introducing to development an enhanced approach for digital watermarking for hiding information that is satisfies these requirements and problems at the same time in an acceptable manner.

Previous methods: The principle previous methods of related works in spatial domain are proposed:

- Wang et al. (2000) proposed hiding data in images by optimal moderately-significant-bit replacement scheme using a genetic algorithm. Instead of embedding the data in the LSB of the cover image, they proposed embedding the data in the moderately-significant-bit $\left(\mathrm{LSB}_{4}\right)$ the fifth bit accounted from left to right hand as shown in Fig. 4 of the cover image. Here, the $\mathrm{LSB}_{4}$ is called the first bit, while the $\mathrm{LSB}_{1}$ is called the eighth bit. With the use of the optimal substitution process by Local Pixel Adjustment Process (LPAP), thus the proposed algorithm: Let $\mathrm{p}$ and p' be the corresponding $(8$ bit) grey values of a pixel of cover image and resulting of embedding image, respectively and $\delta$ be the value of the last three bits (bits 6-8) $\left(\mathrm{LSB}_{1,2,3}\right)$ in p' as shown in Fig. 4. Notice that the max-embedding error in the $\mathrm{LSB}_{4}=2^{4-1}=$ 8. If $\mathrm{p} \neq \mathrm{p}^{\prime}$, then either (i) $\mathrm{p}^{\prime}=\mathrm{p}-8$ or (ii) $\mathrm{p}^{\prime}=\mathrm{p}+8$ (because the only difference between cover image and resulting of embedding image is the fifth bit plane)

Case 1: When $\mathrm{p}^{\prime}=\mathrm{p}-8$. If $\delta \geq 4$, then the value $(8-\delta-1)$ is added to p'. If $\delta<4$ and if the fourth bit of p' is 0 , then the fourth bit of p' is changed to 1 and the value $\delta$ is subtracted from p'. Do nothing otherwise. 


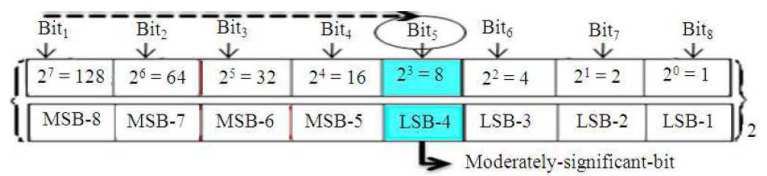

Fig. 4: The one pixel of cover image is converted to the binary bits

Case 2: When $\mathrm{p}^{\prime}=\mathrm{p}+8$. If $\delta<4$, then the value $\delta$ is subtracted from p'. If $\delta \geq 4$ and if the fourth bit of p' is 1 , then the fourth bit of p' is changed to 0 and the value $(8-\delta-1)$ is added to p'. Do nothing otherwise. The image quality of the resulting embedding-image is much better than that of the simple replacement method.

- Chan and Cheng (2004) proposed hiding data in images by simple LSB substitution scheme by applying an Optimal Pixel Adjustment Process (OPAP) to the embedding image obtained by the simple LSB substitution method, then derived the worst case mean-square-error between the embedding image and the cover image. The authors using to embedding the data bits in the $\mathrm{k}$ means capacity of the embedding data bits in the ( $\mathrm{k}$-LSB) of the cover image, where $\mathrm{k}$ given the high capacity of the embedding date bits. They proposed embedding the data in the $\mathrm{LSB}_{1}$ when $\mathrm{k}=1, \mathrm{LSB}_{1,2}$ when $\mathrm{k}=2, \mathrm{LSB}_{1,2,3}$ when $\mathrm{k}=3$ and $\mathrm{LSB}_{1,2,3,4}$ when $\mathrm{k}=4$, to at chive high capacity of embedding data in the $\left(\mathrm{LSB}_{4}\right)$ that a fifth bit accounted from left to right hand as shown in Fig. 4 of the cover image. The authors proposed OPAP: Let $\mathrm{P}_{\mathrm{i}}, \mathrm{P}_{\mathrm{i}}$ and $\mathrm{P}_{\mathrm{i}}{ }_{\mathrm{i}}$ be the corresponding pixel values of the ith pixel in the cover-image $\mathrm{C}$, the embedding-image $\mathrm{C}$ obtained by the simple LSB substitution method and the refined embedding-image obtained after the OPAP. Let $\delta_{\mathrm{i}}=\mathrm{P}^{\prime}{ }^{\prime} \mathrm{P}_{\mathrm{i}}$ be the embedding error between $\mathrm{P}_{\mathrm{i}}$ and $\mathrm{P}_{\mathrm{i}}^{\prime} . \mathrm{P}_{\mathrm{i}}^{\prime}$ is obtained by the direct replacement of the k-LSB of $P_{i}$ with $k$ data bits, therefore, $-2^{k}<\delta_{i}<2^{k}$, the value of $\delta_{\mathrm{i}}$ can be further segmented into three intervals, such that:

Interval 1: $2^{\mathrm{k}-1}<\delta_{\mathrm{i}}<2^{\mathrm{k}}$

Interval 2: $-2^{\mathrm{k}-1} \leq \delta_{\mathrm{i}} \leq 2^{\mathrm{k}-1}$

Interval 3: $-2^{\mathrm{k}}<\delta_{\mathrm{i}}<-2^{\mathrm{k}-1}$

Based on the three intervals, the OPAP, which modifies $\mathrm{P}_{\mathrm{i}}^{\prime}$ to form the embedding pixel $\mathrm{P}_{\mathrm{i}}^{\prime \prime}$, can be described as follows:

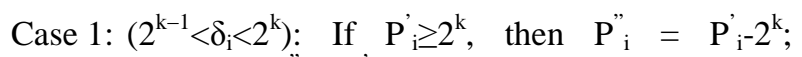
otherwise $\mathrm{P}_{\mathrm{i}}{ }_{\mathrm{i}}=\mathrm{P}_{\mathrm{i}}^{\prime}$
Case 2: $\left(-2^{\mathrm{k}-1} \leq \delta_{\mathrm{i}} \leq 2^{\mathrm{k}-1}\right): \mathrm{P}^{\prime \prime}=\mathrm{P}_{\mathrm{i}}$

Case 3: $\left(-2^{\mathrm{k}}<\delta_{\mathrm{i}}<-2^{\mathrm{k}-1}\right)$ : If $\mathrm{P}_{\mathrm{i}}<256-2^{\mathrm{k}}$, then $\mathrm{P}_{\mathrm{i}}{ }_{\mathrm{i}}=\mathrm{P}_{\mathrm{i}}{ }^{\prime}+2^{\mathrm{k}}$; otherwise $\mathrm{P}_{\mathrm{i}}^{\prime \prime}=\mathrm{P}_{\mathrm{i}}^{\prime}$

where the embedding error between $\mathrm{P}_{\mathrm{i}}$ and $\mathrm{P}_{\mathrm{i}}$ computed by $\delta_{\mathrm{i}}^{\prime}=\mathrm{P}^{\prime}{ }_{\mathrm{i}}-\mathrm{P}_{\mathrm{i}}$. The authors obtained the embedding error after the proposed OPAP is limited to $0 \leq\left|\delta_{\mathrm{i}}\right| \leq 2^{\mathrm{k}-1}$ and computing the Worst PSNR for the capacity of k-data bits by:

$\mathrm{PSNR}_{\text {worst }}=10 \times \log _{10} \frac{255^{2}}{\mathrm{WMSE}}=10 \times \log _{10} \frac{255^{2}}{\left(2^{\mathrm{k}}-1\right)^{2}} \mathrm{~dB}$

where the Worst Mean Square Error $($ WMSE $)=1$ when the k-of-capacity data bits $=1$, WMSE $=3$ when k-ofcapacity data bits $=2$, WMSE $=7$ when k-of-capacity data bits $=3$ and WMSE $=15$ when k-of-capacity data bits $=4$. The authors obtained the $\mathrm{WMSE}^{*}=\left(2^{\mathrm{k}-1}\right)^{2}$ after applying OPAP and by combining the WMSE with the WMSE $^{*}$ after applying OPAP, reveals that:

$\frac{\text { WMSE}^{*}}{\text { WMSE }}=\frac{\left(2^{k-1}\right)^{2}}{\left(2^{k}-1\right)^{2}}$, then $\quad$ WMSE $^{*}=\frac{\left(2^{k-1}\right)^{2}}{\left(2^{k}-1\right)^{2}}$ WMSE

Thus the WMSE ${ }^{*}=0.2844 \mathrm{WMSE}$, when $\mathrm{k}=4$ :

- Aiad and Sada (2007) proposed hiding data using LSB-3 in the cover image. The $\mathrm{LSB}_{3}$ has been used to increase the robustness of the system and protect the data against the external influences for example (noise or compression). The authors using the LPAP by $\mathrm{LSB}_{1,2}$ to modified according to the bit of the data embedded, to minimize the difference between the cover image and the embedding image. Let's have the data bits set $P=\left\{P_{0}, P_{1}, P_{2}\right.$, $\left.\ldots, \mathrm{P}_{\mathrm{L}-1}\right\}$, where $\mathrm{L}$ is the length of the data that is embedded and $\mathrm{P}_{\mathrm{i}}=\{0,1\}$, for $\mathrm{i}=0, . ., \mathrm{L}-1$. Let's have the cover image $=\left\{\operatorname{pixel}_{0,1}, \operatorname{pixel}_{1,1}, \ldots\right.$, $\left.\operatorname{pixel}_{(\mathrm{N}, \mathrm{M})}\right\}$. Suppose that $\mathrm{LSB}_{3}$ of the cover image is $\mathrm{LSB}_{3}=\left\{\mathrm{c}_{0}, \mathrm{c}_{1}, \mathrm{c}_{2}, \ldots, \mathrm{c}_{\mathrm{L}}\right\}$, where $\mathrm{c}_{\mathrm{j}}=\{0,1\}$ for each $\mathrm{I}=0, . . \mathrm{L}$. The embedding process is very easy, which is only replace the permutated bits of the data $\left(P_{i}\right)$ by the $\mathrm{LSB}_{3}$ set of the cover image to obtain the new embedding image $\mathrm{Z}={\text { } \text { newpixel }_{(0,1)} \text {, }}_{\text {, }}$ newpixel $_{(1,1)}, \ldots$, newpixel $\left.l_{(\mathrm{N}, \mathrm{M})}\right\}$. To minimize the difference between the old value (pixel) in the cover image and the new value (newpixel) in the embedding image, the authors propose the following embedding algorithm:

Step 1: Extract $\mathrm{LSB}_{1}$ set of the cover image, $\mathrm{LSB}_{1}=\left\{\mathrm{a}_{0}\right.$, $\left.a_{1}, \ldots, a_{L}\right\}$. //first plane

Step 2: Extract $\mathrm{LSB}_{2}$ set of the cover image, $\mathrm{LSB}_{2}=\left\{\mathrm{b}_{0}\right.$, $\left.\mathrm{b}_{1}, \ldots, \mathrm{b}_{\mathrm{L}}\right\} . / /$ second plane 
Am. J. Applied Sci., 7 (7): 987-1022, 2010

Step 3: For i=1 to L do

$$
\begin{aligned}
& \text { if } \mathrm{p}_{\mathrm{i}}==\mathrm{c}_{\mathrm{i}} \text {, Then do nothing } \\
& \text { else } \\
& \quad \text { if } \mathrm{p}_{\mathrm{i}}==1 \text { and } \mathrm{c}_{\mathrm{i}}==0 \text {, Then } \\
& \mathrm{a}_{\mathrm{i}}=0 ; \mathrm{b}_{\mathrm{i}}=0 \text {; } \\
& \text { else if } \mathrm{p}_{\mathrm{i}}==0 \text { and } \mathrm{c}_{\mathrm{i}}==1 \text {, Then } \\
& \quad \mathrm{a}_{\mathrm{i}}=1 ; \mathrm{b}_{\mathrm{i}}=1 ; \\
& \} ; \\
& \} ; \mathrm{c}_{\mathrm{i}}=\mathrm{p}_{\mathrm{i}} ; \text { embed data bit in the } \mathrm{LSB}_{3} \text { of the cover }
\end{aligned}
$$

The authors explained the above algorithm; let's have the following pixel in the cover image, pixel = $(3)_{10}=(00000011)_{2}$. Suppose we need to embed $p=1$ in the LSB-3, so the new pixel will be, newpixel = $(00000111)_{2}=(7)_{10}$. Notice that the difference is $7-3=$ 4. In embedding algorithm, The authors say will set $\mathrm{LSB}_{1,2}$ to 0 when $\mathrm{p}=1$ and $\mathrm{c}=0$. So newpixel $=$ $(00000100)_{2}=(4)_{10}$. Where the deference is becomes 4$3=1$. On the other hand, suppose that pixel $=(4)_{10}=$ $(00000100)_{2}$ and $\mathrm{p}=0$. The newpixel $=(00000000)_{2}=$ $(0)_{10}$. The difference is $4-0=4$. The embedding algorithm, in this case will be set $\mathrm{LSB}_{1,2}$ to ' 1 ', so newpixel $=(00000011)_{2}=(3)_{10}$. Where the difference is becomes $4-3=1$. Thus the differences in the $\mathrm{LSB}_{3}$ replacement are less or equal one as in the $\mathrm{LSB}_{1}$ but in more robust.

Kevin et al. (2005) proposed an investigation into the use of the least significant bit substitution technique in digital watermarking, study presents the results of implementing a LSB in digital watermarking system to investigation the digital watermarking is used by those who wish to prevent others from stealing their material. The authors say the LSB substitution is not a very good candidate for digital watermarking, but it is very useful in the art of steganography, due to its lack of robustness. The LSB embedded watermarks can be easily recovered and even altered by an attacker. Otherwise if the watermark is hidden in the LSB, all the individual has to do is flip one LSB and the information cannot be recovered. It would appear that LSB will remain in the domain of steganography due to its useful nature and its overall capacity of information. Where image Steganography, in the LSB substitution, the least significant bit is changed because this has little effect to the appearance of the carrier message. This shows that the gray scale image would change significantly if there were any other bit changed than the LSB. It changes more and more the closer you get to the Most Significant Bit. When the LSB is changed, the pixel bit value changes from 128-129, which is undetectable with the human eye. With the MSB changed, the pixel bit value changes from 128-0, which makes high a significant change to the gray scale view. The theory is that if you take two gray scale images and change the LSB of image one to the LSB of image two for each coordinate or pixel, image two will be hidden in image as (changes from 128-129), then the embedded of massage bit in image pixel (129) in the first LSB, there should be no detectable change or alteration to the appearance of the first image pixel (128). Otherwise there are a variety of digital carriers or places where data can be hidden. Data may be embedded in files at imperceptible levels of noise and properties of images can be changed and used in a way useful to your aim. The authors study focuses on bit values of pixel in the gray scale range which can be altered to embed hidden images inside other images, without changing the actual appearance of the carrier image. While the watermarking is the process of hiding information in a carrier in order to protect the ownership of image, text, music, films and art, where watermarking can be used to hide or embed visible or hidden copyright information. Watermarking does not impair the image. This is a main concern with visible watermarking. Even though the watermark can be seen, it must be inserted in such a way that it does not interfere with the original image with an invisible watermark you can change certain pixels in an image so the human eye cannot tell the difference from the original image. The important properties of watermarking are perceptual transparency, robustness, security and payload. Finally the authors concluded the LSB substitution is not a very good candidate for digital watermarking techniques.

Recently, a new digital watermarking technique robust and oblivious digital watermarking image in spatial domain (Mehemed et al., 2009) capable of embedding a totally indistinguishable in original image by the human eye by applying Falling-Off-Boundary in Corners Board of cover image (FOBCB) with the random pixel manipulation set of the Most-SignificantBit-6 ( $\mathrm{MSB}_{6}$ ) (the bits are accounted from right to left hand) as shown in Fig. 5 is developed to improve the quality of embedding results, imperceptibility, undetectability and robustness. Whereas the binary watermark insertion process needs the secret $\mathrm{Ke}_{\mathrm{y} 1}$ to determine the number of frames per row in watermark and secret $\mathrm{Ke}_{\mathrm{y} 2}$ to changing the pixels of watermark depending on the number of frames per row determined by secret $\mathrm{Ke}_{\mathrm{y} 1}$.

Step 1: One watermark pixel is inserted in each of FOBCB of cover image with random pixel manipulation set of the $\mathrm{MSB}_{6}$. Before insertion will be using secret key for spatial dispersion of the watermark to rearranging pixels as the following below: First: Reads the indexed of watermark W into X. 
Am. J. Applied Sci., 7 (7): 987-1022, 2010

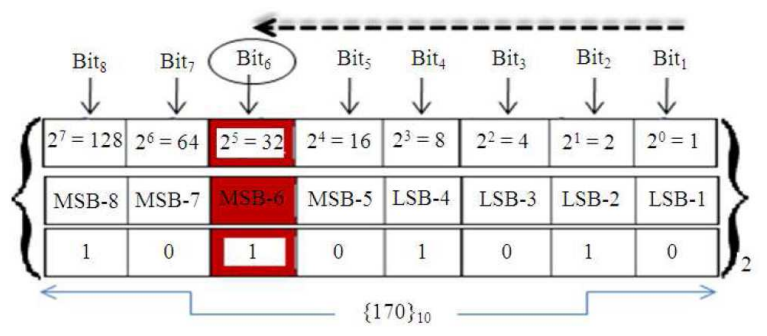

Fig. 5: One pixel of cover image is converted to the binary bits $\left(\operatorname{LSB}_{(1,2,3,4)}\right.$ and $\left.\operatorname{MSB}_{(5,6,7,8)}\right)$

From the indexed identify the size of matrix X[W, L]. Second: Secret Key ${ }_{1}$ using to determine the number of frames per row, where the Key ${ }_{1}$ chosen the dimension number divided by the frame number without remainder as Eq. 1a and 1b:

$\mathrm{m}=$ width $(\mathrm{w}) /$ number of frames $\left(\mathrm{Key}_{1}\right)$

$\mathrm{n}=$ length (1) / number of frames $\left(\mathrm{Key}_{1}\right)$

Third: Define the indexed identify the size of new matrix $\mathrm{Y}[\mathrm{W}, \mathrm{L}]$ for the rearranging pixels of the watermark and then using $\mathrm{Key}_{2}$ to generate the random permutation of the integers depending on the number of Secret Key ${ }_{1}$ :

$\operatorname{Key}_{2}=\left(1:\right.$ number of frames per row $\left.\left(\operatorname{Key}_{1}\right)\right)$

Four: Generate two loops [i, j] to selecting a frames by secret $\mathrm{Key}_{1}$ from indexed identify the size of matrix $\mathrm{X}[\mathrm{W}, \mathrm{L}]$ and by defined the indexed identify the size of new matrix $\mathrm{Y}[\mathrm{W}, \mathrm{L}]$ to changing pixels of watermark depending on the secret $\mathrm{Key}_{2}$ as the algorithm below:

Algorithm:

For $\mathrm{i}=1$ to Secret $\mathrm{Key}_{1}$ do

For $\mathrm{j}=1$ to Secret $\mathrm{Key}_{1}$ do

Selecting the frames from indexed identify the size of matrix X[W, L] by Secret Key ${ }_{1}$ in to matrix WK.

$W K=(X[(i-1) \times m+1: i \times m,(j-1) \times n+1: j \times n])$

From Eq. 1d by arranging the frames of pixels by using Secret $\mathrm{Key}_{2}$ to changing the selecting frames in the new image $\mathrm{Y}[\mathrm{]}$ as Eq. 1e:

$\mathrm{Y}\left[\left(\mathrm{Key}_{2}(\mathrm{i})-1\right) \times \mathrm{m}+1: \mathrm{Key}_{2}(\mathrm{i}) \times \mathrm{m}\right.$, $\left.\left(\mathrm{Key}_{2}(\mathrm{j})-1\right) \times \mathrm{n}+1: \mathrm{Key}_{2}(\mathrm{j}) \times \mathrm{n}\right]=\mathrm{WK} \rightarrow$

\}$;\}$.
Five: For more robustness in digital watermarking applying drawbacks of the payload of watermark in the FOBCB are placed in more than one place in the cover image to prevent the blurring attacks to alter it and cannot defeat the purpose, as the algorithm below:

For ii $=1$ to $\mathrm{T}$ do

For $\mathrm{jj}=1$ to $\mathrm{U}$ do

$\operatorname{Drawback}(\mathrm{ii}, \mathrm{jj})=\operatorname{payload}(\bmod (\mathrm{ii}, \mathrm{T})+1, \bmod (\mathrm{jj}, \mathrm{U})+1)$; \}$;\}$ where is the size of drawbacks [T, U].

Step 2: In this published method, the cover image is of size $[\mathrm{M}, \mathrm{N}] 512 * 512$ gray scale image has been used. In this scheme hide a payload of watermark up to 2025 bits. Embedding payload of watermark in FOBCB of cover image with random pixel manipulation between boundary corners board set of the $\mathrm{MSB}_{6}$. Let's have the drawbacks payload bits set of the $\mathrm{WL}_{(\mathrm{ii}, \mathrm{j} j \mathrm{j}}$, the max-bits can be embedded $1 \leq \mathrm{T} \times \mathrm{U} \leq 2025$ bits, whereas the size of $\mathrm{WL}=[\mathrm{T}, \mathrm{U}]$ and $\mathrm{T}$ equal $\mathrm{U}$. Let's have the cover image $\mathrm{F}=\left\{\right.$ pixel $\left._{0}, \operatorname{pixel}_{1}, \ldots, \operatorname{pixel}_{262144}\right\}$. So, has been determine the pixels of FOBCB of cover image employed as a sequence number $\mathrm{k}_{1}, \mathrm{k}_{2}, \mathrm{k}_{3}, \mathrm{k}_{4}$ where $\mathrm{k}_{1}=1,2, \ldots, \mathrm{N}, \mathrm{k}_{2}=1,2, \ldots, \mathrm{N}, \mathrm{k}_{3}=2,3, \ldots ., \mathrm{M}-1$ and $\mathrm{k}_{4}=2,3, \ldots, \mathrm{M}-1$, then employed sequence number $\mathrm{G}$ to manipulation of pixel between boundary corners board in cover image where $1 \leq \mathrm{G} \leq 4$, as the following embedding algorithm:

\section{Embedding algorithm:}

For ii $=1$ to size of drawback

For $\mathrm{jj}=1$ to size of drawback

if $\mathrm{G}==1$ Then do

if $\mathrm{k}_{1}<=\mathrm{N}$, then do get the corner pixel in FOBCB when $\mathrm{F}\left(1, \mathrm{k}_{1}\right)$ and set bit of the $\mathrm{MSB}_{6}$, then $\mathrm{f}\left(1, \mathrm{k}_{1}\right)=$ embedded the payload of watermark bit $\mathrm{WL}_{(\mathrm{i}, \mathrm{ij})}$ to $\mathrm{MSB}_{6}$ of the pixel $\mathrm{F}\left(1, \mathrm{k}_{1}\right)$ $\mathrm{k}=\mathrm{k}_{1}+1$;

\}; \}

if $\mathrm{G}==2$

if $\mathrm{k}_{2}<=\mathrm{N}$, then get the corner pixel in FOBCB when $\mathrm{F}\left(\mathrm{M}, \mathrm{k}_{2}\right)$ and set bit of the $\mathrm{MSB}_{6}$, then $\mathrm{f}(\mathrm{M}$, $\mathrm{k}_{2}$ ) = embedded the payload of watermark bit $\mathrm{WL}_{(\mathrm{ii}, \mathrm{jj})}$ to $\mathrm{MSB}_{6}$ of the pixel $\mathrm{F}\left(\mathrm{M}, \mathrm{k}_{2}\right)$ $\mathrm{k}_{2}=\mathrm{k}_{2}+1$

\}$;\}$

if $\mathrm{G}==3$

if $\mathrm{k}_{3} \sim=\mathrm{M}$, then get the corner pixel in FOBCB when $\mathrm{F}\left(\mathrm{k}_{3}, 2\right)$ and set bit of $\mathrm{MSB}_{6}$, then $\mathrm{f}\left(\mathrm{k}_{3}, 2\right)=$ embedded the payload of watermark bit $\mathrm{WL}_{(\mathrm{i}, \mathrm{ij})}$ to $\mathrm{MSB}_{6}$ of the pixel $\mathrm{F}\left(\mathrm{k}_{3}, 2\right)$ 


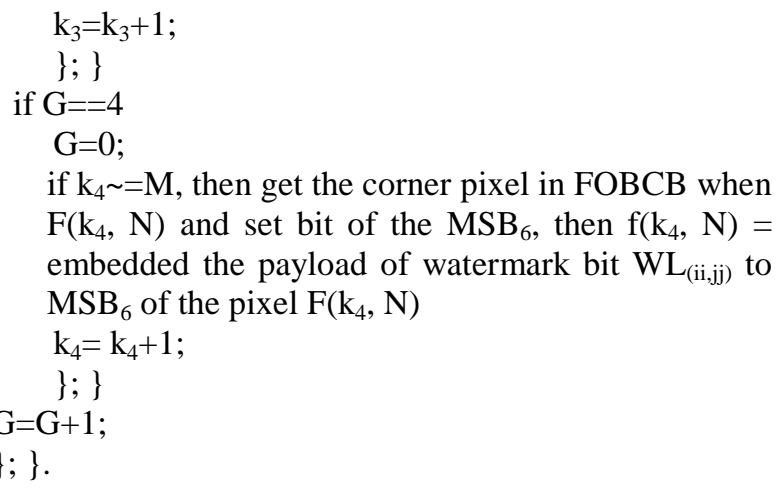

The algorithm protects the payload bits by sequence number? This sequence of indexes used to permute the payload bits. The embedding process is very easy to a achieve the low complexity time, which is only replace the permutated bits of the payload by the $\mathrm{MSB}_{6}$ set of the FOBCB in cover image with random pixel manipulation between boundary corners board obtain the new digital watermarking $\mathrm{f}_{(\mathrm{M}, \mathrm{N})}=\left\{\right.$ newpixel $_{0}$, newpixel $_{1}, \ldots$, newpixel $\left.{ }_{262144}\right\}$.

Step 3: Reconstruct the watermark using to extracted watermark bits from drawbacks in FOBCB of digital watermarking $\mathrm{f}_{(\mathrm{M}, \mathrm{N})}$ by using inverse the same procedure of embedded algorithm with sequence number $G$ to know the manipulation pixel between boundary corners board in digital watermarking $\mathrm{f}_{(\mathrm{M}, \mathrm{N})}$, then select one of drawbacks set of $\mathrm{MSB}_{6}$, after extracted watermark required the secret $\mathrm{Key}_{1,2}$ to rearranging the frames per row, then watermark in original form is thus obtained. This is completes watermark extraction process.

\section{MATERIALS AND METHODS}

The materials and methods are used to measure the performance of watermarking system by applying the benchmark of watermarked image under the requirements of digital watermarking as: (Imperceptibility measured by (average absolute difference, normalized average absolute difference, mean square error, normalized mean square error, signal to noise ratio and peak signal to noise ratio), Image Fidelity and Robustness measured under :(watermark degrading attacks, watermark removal attacks and geometric transformations attack):

Imperceptibility: Means that the perceived quality of the host image should not be distorted by the presence of the watermark (Ali, 2007). Developers and implementers of watermarking image need a standard metric to measure the quality of watermarked images compared with the original image. Thus will be lists the most popular difference distortion measures of pixel based metrics (Lu, 2005). These measures metrics are all based on the difference between the original images and undistorted or the modified image (watermarked image). The mathematical formulae for the list of pixel based metrics are:

Average absolute difference: The $\mathrm{AD}$ is used as the dissimilarity measurement between original image $\mathrm{F}_{(\mathrm{M}, \mathrm{N})}$ and watermarked image $\mathrm{f}_{(\mathrm{M}, \mathrm{N})}$ to enhancement the watermarked image. Whereas a lower value of $\mathrm{AD}$ signifies lesser error in the watermarked image:

$$
\mathrm{AD}=\frac{1}{\mathrm{M} \times \mathrm{N}} \sum_{\mathrm{i}=1}^{\mathrm{M}} \sum_{\mathrm{j}=1}^{\mathrm{N}}\left|\mathrm{F}_{(\mathrm{i}, \mathrm{j})}-\mathrm{f}_{(\mathrm{i}, \mathrm{j})}\right|
$$

Normalized average absolute difference: The NAD is quantization error for any single pixel in the image. This distance measure is normalized to a range between 0 and 1 :

$$
N A D=\frac{\sum_{i=1}^{M} \sum_{j=1}^{N}\left|F_{(i, j)}-f_{(i, j)}\right|}{\sum_{i=1}^{M} \sum_{j=1}^{N}\left|F_{(i, j)}\right|}
$$

Mean square error: The MSE is the cumulative squared error between the watermarked image $\mathrm{f}_{(\mathrm{M}, \mathrm{N})}$ and the original image $\mathrm{F}_{(\mathrm{M}, \mathrm{N})}$ (Satish Kumar, 2001). Moreover the MSE measures the error with respect to the centre of the image values, i.e., the mean of the pixel values of the image and by averaging the sum of squares of the error between the two images (Bijay et al., 2005). A lower value of MSE signifies lesser error in the watermarked image (Satish Kumar, 2001; Bijay et al., 2005):

$\operatorname{MSE}=\frac{1}{M \times N} \sum_{i=1}^{M} \sum_{j=1}^{N}\left(F_{(i, j)}-f_{(i, j)}\right)^{2}$

Normalized mean square error: The normalized mean quantization square error for any single pixel in the image. This distance measure is normalized to a range between 0 and 1 . It is independent of the range of gray scale values in the image, adapted from (GraphicsMagick Group, 2002):

$$
\text { NMSE }=\frac{\sum_{i=1}^{M} \sum_{j=1}^{N}\left(F_{(i, j)}-f_{(i, j)}\right)^{2}}{\sum_{i=1}^{M} \sum_{j=1}^{N} F_{(i, j)}{ }^{2}}
$$


Signal to noise ratio: The SNR is a measure used to quantify how much a signal has been corrupted by noise. It is defined as the ratio of signal power (original image) to the noise power corrupting the signal (embedding errors between original image and watermarked image). A ratio higher than 1:1 indicates more signal than noise, adapted from (Wikipedia, 2010a: The Cooke Corporation Kelheim, 2007):

$$
\begin{gathered}
\text { The singnal power }=\sum_{\mathrm{i}=1}^{\mathrm{M}} \sum_{\mathrm{j}=1}^{N} \mathrm{~F}_{(\mathrm{i}, \mathrm{j})}{ }^{2} \\
\text { The noise power corrupting the signal }=\sum_{\mathrm{i}=1}^{\mathrm{M}} \sum_{\mathrm{j}=1}^{\mathrm{N}}\left(\mathrm{F}_{(\mathrm{i}, \mathrm{j})}-\mathrm{f}_{(\mathrm{i}, \mathrm{j})}\right)^{2} \\
\operatorname{SNR}=\frac{\sum_{\mathrm{i}=1}^{\mathrm{M}} \sum_{\mathrm{j}=1}^{N} \mathrm{~F}_{(\mathrm{i}, \mathrm{j})}{ }^{2}}{\sum_{\mathrm{i}=1}^{M} \sum_{\mathrm{j}=1}^{N}\left(\mathrm{~F}_{(\mathrm{i}, \mathrm{j})}-\mathrm{f}_{(\mathrm{i}, \mathrm{j})}\right)^{2}}
\end{gathered}
$$

They are usually measured in decibels (dB) (Kamran et al., 2006; Muhammad and Dot, 2003): SNR $(\mathrm{dB})=10 \times \log 10(\mathrm{SNR}):$

$\operatorname{SNR}=10 \times \log _{10}\left(\frac{\sum_{\mathrm{i}=1}^{\mathrm{M}} \sum_{\mathrm{j}=1}^{\mathrm{N}} \mathrm{F}_{(\mathrm{i}, \mathrm{j})}{ }^{2}}{\sum_{\mathrm{i}=1}^{\mathrm{M}} \sum_{\mathrm{j}=1}^{\mathrm{N}}\left(\mathrm{F}_{(\mathrm{i}, \mathrm{j})}-\mathrm{f}_{(\mathrm{i}, \mathrm{j})}\right)^{2}}\right) \mathrm{dB}$

Notice that the higher ratio means the less obtrusive of the embedding errors (noise) in the watermarked image, whereas the SNR is a technical term used to characterize the quality of the watermarked image detected of a measuring watermark system are adapted from (Wikipedia, 2010b).

Peak signal to noise ratio: The PSNR computes, in decibels, between two images (Ali, 2007). This ratio is often used as a quality measurement between the original and a watermarked image, adapted from (MathWorks Inc, 2010). The higher the PSNR is the better the quality of the watermarked image (Bijay et al., 2005; Wang, 2006) and is a standard way to measure image fidelity (Bijay et al., 2005). The PSNR is derived by setting the MSE in relation to the maximum possible value of the luminance (for a typical 8-bit value this is $2^{8}-1=255$ ), adapted from (MathWorks Inc, 2010) as follows:

$$
\mathrm{PSNR}=10 \log _{10} \frac{255^{2}}{\mathrm{MSE}} \mathrm{dB}
$$

Notice that the MSE and the PSNR are the two error metrics used to compare watermarking image quality, adapted from (MathWorks Inc, 2010). Also the PSNR can be computed with the Root Mean Squared Error (RMSE) (Kamran et al., 2006; Muhammad and Dot, 2003). The RMSE is the square root of mean square error. It quantifies the average sum of distortion in each pixel of the reconstructed image (watermarking image) (Bijay et al., 2005):

$$
\text { PSNR }=20 \log _{10} \frac{255}{\text { RMSE }} \mathrm{dB}
$$

Whereas a lower value for MSE means lesser error and as seen from the inverse relation between the MSE and PSNR, this translates to a high value of PSNR. Logically, a higher value of PSNR is good because it means that the ratio of signal to noise is higher. Here, the 'signal' is the original image and the 'noise' is the embedding errors of embedded watermark bits in cover image. So, if you find an embedding watermark scheme having a lower MSE (and a high PSNR), you can recognize that it is a better one, adapted from (Satish Kumar, 2001).

Image Fidelity (IF): Refers to the closeness of a watermarked image to a reference of original image (Lu, 2005). On the other hand the image fidelity how closely the image represents the real source distribution depends not only on random noise but also on errors in the data, sampling and image artifacts. Thus is a comparative measure of the distance between a pair of images:

$$
\mathrm{IF}=\frac{1-\sum_{\mathrm{i}=1}^{M} \sum_{\mathrm{j}=1}^{N}\left(\mathrm{~F}_{(\mathrm{i}, \mathrm{j})}-\mathrm{f}_{(\mathrm{i}, \mathrm{j})}\right)^{2}}{\sum_{\mathrm{i}=1}^{\mathrm{M}} \sum_{\mathrm{j}=1}^{N} \mathrm{~F}_{(\mathrm{i}, \mathrm{j})}{ }^{2}}
$$

However, that Image fidelity in Eq. 2i does not provide an adequate measure of perceived image fidelity, Thus that an image fidelity measure is also commonly referred to as an image metric, the traditional image fidelity standard is the MSE, SNR and the PSNR for the original image with watermarked image. Nowadays, the most popular distortion measures in the field of image or a common measure used of the quality of a watermarked image $\mathrm{f}_{(\mathrm{i}, \mathrm{j})}$ and compression are the SNR, MSE and PSNR is typically used. It is familiar to workers in the field, it is also simple to 
calculate, but it has only a limited, approximate relationship with the perceived errors noticed by the human visual system. This is why higher PSNR values imply closer resemblance between the watermarking image $f_{(i, j)}$ and the original images $F_{(i, j)}$. Denoting the pixels of the original image by $F_{(i, j)}$ that contains ( $M$ by $\mathrm{N})$ pixels represent the size of image $(\mathrm{M}, \mathrm{N}$ are the dimensions of the images) and the pixels of the image watermarking image by $f_{(i, j)}$, where $f$ is reconstructed watermarking image by decoding the encoded version of original image $\mathrm{F}_{(\mathrm{i}, \mathrm{j})}$. Error metrics are computed on the luminance value only so the pixel values $\mathrm{f}_{(\mathrm{i}, \mathrm{j})}$ in the range between black (0) and white (255) (Katzenbeisser and Petitcolas, 1999; David et al., 2007). For a gray scale image with eight bits per pixel, the numerator is 255 . For color images, only the luminance component is used. The typical PSNR values range between 20 and 40 (Eric, 2003; David et al., 2007). Some definitions of PSNR use Eq. 2 g) rather than Eq. 2h. Either formulation will work because we are interested in the relative comparison, not the absolute values. For our proposal we will use the definition given above in Eq. $2 \mathrm{~g}$.

Robustness: It is a measure of the immunity of the watermark against attempts to remove or degrade it, intentionally or unintentionally, by different types of digital signal processing attacks (Lin, 2005). We will report on robustness results which we obtained of major attacks:

- Watermark degrading attacks: Gaussian noise, Salt and Pepper noise and Speckle noise (Kamran et al., 2006; Muhammad and Dot, 2003)

- Watermark removal attacks: Changing in lower order bit manipulation of gray scale values $\mathrm{LSB}_{1,2,3,4}$, Altered image and Drawing image (Yusnita and Khalifa, 2008)

- Geometric transformations attack (Bijay et al., 2005; Martin and Petitcolas, 2000; Kutter and Petitcolas, 1999; Fernando and Hernandez, 1999): Image cropping (Lin, 2005): In some cases, infringers are just interested by the "central" part of the copyrighted material. Scaling (Santi and Kundu, 2002; Matt et al., 1999): It can be divided into two groups, uniform and non-uniform scaling. Under uniform scaling we understand scaling which is the same in horizontal and vertical direction. Non-uniform scaling uses different scaling factors in horizontal and vertical direction. Lossy data compression like JPEG (Santi and Kundu, 2002; Lin, 2005): JPEG is currently one of the most widely used compression algorithms for images and any watermarking system should be resilient to some degree of compression. Rotation: Small angle rotation (Martin and Petitcolas, 2000; Kutter and Petitcolas, 1999). Finally: Horizontal flip (Martin and Petitcolas, 2000; Kutter and Petitcolas, 1999; Bijay et al., 2005)

They are good representatives of the more general attacks. We measured the similarity between the original watermark $\mathrm{W}_{(\mathrm{i}, \mathrm{j})}$ and the watermark extracted $\mathrm{W}^{\prime}{ }_{(\mathrm{i}, \mathrm{j})}$ from the attacked image, whereas the similarity values NCC and SM of about 0.75 or above is considered acceptable (Ali, 2007; Kamran et al., 2006; Muhammad and Dot, 2003).

Normalized cross correlation: The quantitative estimation for the quality of extracted watermark image $\mathrm{W}_{(\mathrm{i}, \mathrm{j})}$ with reference to the original watermark $\mathrm{W}_{(\mathrm{i}, \mathrm{j})}$ can be expressed as normalized cross correlation gives maximum value of (NCC) as unity defined as (Katzenbeisser and Petitcolas, 1999; Lu, 2005):

$$
\mathrm{NCC}=\frac{\sum_{\mathrm{i}=1}^{\mathrm{M}} \sum_{\mathrm{j}=1}^{\mathrm{N}}\left(\mathrm{W}_{(\mathrm{i}, \mathrm{j})} \times \mathrm{W}_{(\mathrm{i}, \mathrm{j})}^{\prime}\right)}{\sum_{\mathrm{i}=1}^{\mathrm{M}} \sum_{\mathrm{j}=1}^{\mathrm{N}} \mathrm{W}_{(\mathrm{i}, \mathrm{j})}{ }^{2}}
$$

Similarity function: Function similarity estimation between extracted watermark $\mathrm{W}_{(\mathrm{i}, \mathrm{j})}^{\prime}$ and original watermark $\mathrm{W}_{(\mathrm{i}, \mathrm{j})}$ is computed by the following formula (Lin, 2005):

$$
\mathrm{SM}=\frac{\sum_{\mathrm{i}=1}^{\mathrm{M}} \sum_{\mathrm{j}=1}^{\mathrm{N}}\left(\mathrm{W}_{(\mathrm{i}, \mathrm{j})} \times \mathrm{W}_{(\mathrm{i}, \mathrm{j})}\right)}{\sqrt{\sum_{\mathrm{i}=1}^{\mathrm{M}} \sum_{\mathrm{j}=1}^{\mathrm{N}} \mathrm{W}_{(\mathrm{i}, \mathrm{j})}{ }^{2} \times \sum_{\mathrm{i}=1}^{\mathrm{M}} \sum_{\mathrm{j}=1}^{\mathrm{N}} \mathrm{W}^{\prime}{ }_{(\mathrm{i}, \mathrm{j})}^{2}}}
$$

If the result is larger than some determined threshold, we consider the extracted watermark $\mathrm{W}_{(\mathrm{i}, \mathrm{j})}$ are equal original watermark $\mathrm{W}_{(\mathrm{i}, \mathrm{j})}$.

Study with analysis and modified the previous methods: To study the performance and comparisons between the state-of-the-art algorithms will be modified the algorithms of Pixel Adjustment Process (PAP) are based in the LSB techniques proposed in (Kevin et al., 2005; Chan and Cheng, 2004; Aiad and Sada, 2007; Wang et al., 2000), after that will be applying the modification algorithms of PAP by the our embedding algorithm of the FOBCB set-of-the Most-SignificantBit-6 (MSB $\left.{ }_{6}\right)$ is published in (Mehemed et al., 2009), reviewed in previous methods. Notice that the maximum of embedding errors in the $\mathrm{MSB}_{6}$ by directly 
replacement of embedding watermark bits $=2^{\mathrm{n}-1}=32$ as shown in Fig. 5.

Moreover there is a trade-off between the embedding errors in the LSB and MSB, where the embedding errors are growth up as shown in Fig. 5. Let's have the binary watermark image $\mathrm{WL}_{(\mathrm{ii}, \mathrm{j} j}$, with the size of $\mathrm{WL}=[\mathrm{T}, \mathrm{U}], \mathrm{WL}=\{0,1\}$ and the cover image $\mathrm{F}=\left\{\right.$ pixel $\left._{1}, \operatorname{pixel}_{2}, \ldots, \operatorname{pixel}_{(\mathrm{M} \times \mathrm{N})}\right\}=\mathrm{P}_{(\mathrm{i}, \mathrm{j})}$, after extracted the pixels from cover image, will be converted the cover image pixels $\mathrm{P}_{(\mathrm{i}, \mathrm{j})}$ in to the binary numbers ( 8 bits grey values par pixel), then set of the Most-Significant-Bit $\left(\mathrm{MSB}_{6}\right)$ in each pixel of the cover image $\mathrm{P}_{(\mathrm{i}, \mathrm{j})}$ as shown in Fig. 5 accounted from right to the left hand, the following modified algorithms in the $\mathrm{MSB}_{\mathrm{n}}$, where is $\mathrm{n}$ equal 6 .

PAP-algorithm-1: We modified the scheme of Wang et al. (2000) using a Local Pixel Adjustment Process (LPAP) the proposed algorithm used $\mathrm{LSB}_{4}$ for embedding data bits, thus will be modified the algorithm of LPAP on the Most Significant Bit-6 $\left(\mathrm{MSB}_{6}\right)$. However the embedding error in $\mathrm{MSB}_{6}$ equal 32 was trade-off with the embedding error in $\mathrm{LSB}_{4}$ equal 8. Let $\mathrm{P}_{(\mathrm{i}, \mathrm{j})}$ and $\mathrm{p}_{(\mathrm{i}, \mathrm{j})}$ be the corresponding 8 bit grey values of a pixel in the cover image as shown in Fig. 5 and $\mathrm{p}_{(\mathrm{i}, \mathrm{j})}$ watermarked image obtained by the FOBCB set of Most-Significant-Bit-6 (MSB $\left.{ }_{6}\right)$ scheme, respectively and $\delta$ be the value of the ( $\mathrm{LSB}_{1,2,3,4}$ and $\left.\mathrm{MSB}_{5}\right)$ as well as from $\left\{\mathrm{bit}_{1}\right.$ to $\left.\mathrm{bit}_{5}\right\}$ in $\mathrm{p}_{(\mathrm{i}, \mathrm{j})}$ as shown in Fig. 2.

If $\mathrm{P}_{(\mathrm{i}, \mathrm{j})} \neq \mathrm{p}_{(\mathrm{i}, \mathrm{j})}$, then either (i) $\mathrm{p}_{(\mathrm{i}, \mathrm{j})}=\mathrm{P}_{(\mathrm{i}, \mathrm{j})}-2^{\mathrm{n}-1}$ or (ii) $\mathrm{p}_{(\mathrm{i}, \mathrm{j})}^{\prime}=\mathrm{P}_{(\mathrm{i}, \mathrm{j})}+2^{\mathrm{n}-1}$ (because the only difference between cover image and resulting of embedding image is the six bit plane $\left(\mathrm{MSB}_{6}\right)$ ).

Case 1: when $\mathrm{p}_{(\mathrm{i}, \mathrm{j})}^{\prime}=\mathrm{P}_{(\mathrm{i}, \mathrm{j})}-2^{\mathrm{n}-1}$. If $\delta \geq 2^{\mathrm{n}-2}$, then the value $\left(2^{\mathrm{n}-1}-\delta-1\right)$ is added to $\mathrm{p}^{\prime}(\mathrm{i}, \mathrm{j})$. If $\delta<2^{\mathrm{n}-2}$ and if the seven bit of $p_{(i, j)}^{\prime}$ is zero, then the seven bit of $p^{\prime}{ }_{(i, j)}$ is changed to one and the value $\delta$ is subtracted from $\mathrm{p}^{\prime}(\mathrm{i}, \mathrm{j})$. Do nothing otherwise.

Case 2: when $\mathrm{p}_{(\mathrm{i}, \mathrm{j})}=\mathrm{P}_{(\mathrm{i}, \mathrm{j})}+2^{\mathrm{n}-1}$. If $\delta<2^{\mathrm{n}-2}$, then the value $\delta$ is subtracted from $\mathrm{p}_{(\mathrm{i}, \mathrm{j})}$. If $\delta \geq 2^{\mathrm{n}-2}$ and if the seven bit of $\mathrm{p}_{(\mathrm{i}, \mathrm{j})}$ is one, then the seven bit of $\mathrm{p}_{(\mathrm{i}, \mathrm{j})}$ is changed to zero and the value $\left(2^{\mathrm{n}-1}-\delta-1\right)$ is added to $\mathrm{p}_{(\mathrm{i}, \mathrm{j})}$. Do nothing otherwise.

Analysis: Notice that from the PAP of the Wang-LinLin scheme, we know that only the first three bits (bits 1-3) and the five bit $\left(\mathrm{MSB}_{5}\right)$ as shown in Fig. 2 are modified to improve the image quality. It is obvious that the algorithm is not optimal by analysis the possibility values of gray scale image as shown in Fig. 6.

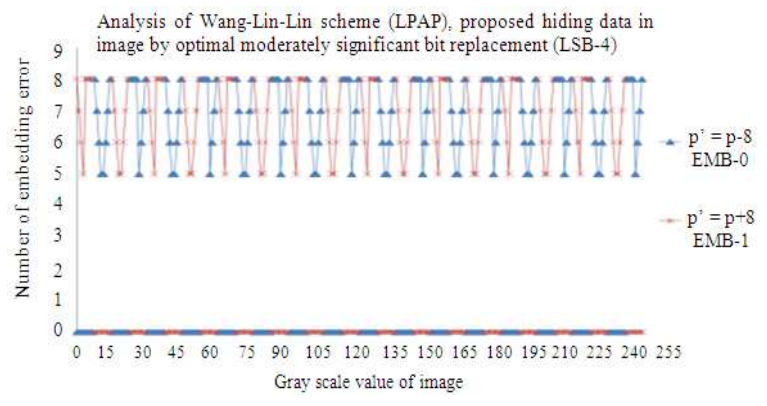

Fig. 6: The embedding error of proposed Wang-Lin-Lin scheme

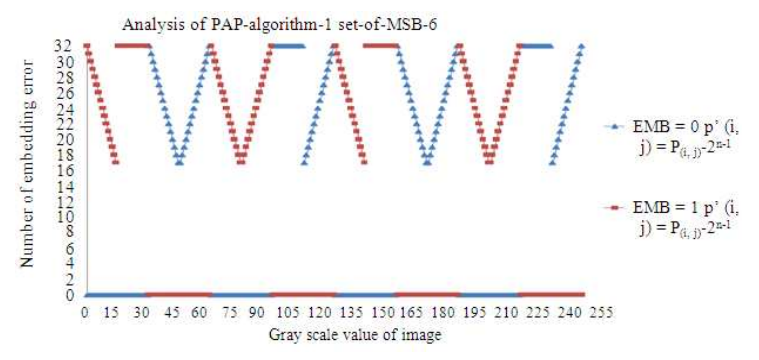

Fig. 7: The embedding errors of modified scheme (PAP-algorithm-1)

While if $\mathrm{P}_{(\mathrm{i}, \mathrm{j})}=8,15,24,25,26,27,31,40,248$, 249, 250 and 251 when the embedded watermark bit equal zero and when $\mathrm{P}_{(\mathrm{i}, \mathrm{j})}=0,4,5,6,7,16,23,31,32$, 228, 229, 230, 231 and 240 with the embedded watermark bit equal one, whereas the embedding error go to level of maximum error in the $\mathrm{LSB}_{4}=2^{\mathrm{n}-1}=8$, for example if $\mathrm{P}_{(\mathrm{i}, \mathrm{j})}=(31)_{10}(00011111)_{2}$, then the $\mathrm{LSB}_{4}$ in cover image is ' 1 '. If the embedded watermark bit is ' 0 ' using the Wang-Lin-Lin scheme, we have p' $=(23)_{10}$ $(00010111)_{2}$ as well as the embedding error for the modification is $=|31-23|=8$ as the same of listed pixel before as shown in Fig. 6. However, it can be seen that the embedding error go to the minimum error if $\mathrm{P}_{(\mathrm{i}, \mathrm{j})}=$ 11, 12, 28, 60, 75, 76, 92, 107, 108, 124, 235, 236 and 252 when the embedded watermark bit equal zero and $\mathrm{P}_{(\mathrm{i}, \mathrm{j})}=3,19,20,35,51,52,67,83,84,99,115,116$, 243 and 244 when the embedded watermark bit equal one; as well as the embedding error go to the minimum level error in $\operatorname{LSB}_{4}=\left(2^{\mathrm{n}-2}+1\right)=5$, as shown in the analysis result in Fig. 6. Also the same problem of modified scheme (PAP-algorithm-1), when applying the algorithm in $\mathrm{MSB}_{6}$ as showed the analysis result in Fig. 7. Also it is obvious that the modification is not optimal where the embedding error are confined between the maximum level of the embedding error in $\operatorname{MSB}_{6}=\left(2^{\mathrm{n}-1}\right)=32$ and in the minimum level of the embedding error in $\mathrm{MSB}_{6}=\left(2^{\mathrm{n}-2}+1\right)=17$. Thus the 
observation of the analysis result in the both algorithms $\left(\mathrm{LSB}_{4}\right.$ and $\left.\mathrm{MSB}_{6}\right)$; where the embedding error are growth up one by one start from $\left(2^{\mathrm{n}-2}+1\right)$ to the maximum of embedding error $\left(2^{\mathrm{n}-1}\right)$ and then go down to the minimum of the embedding error $\left(2^{\mathrm{n}-2}+1\right)$ and growth up again, when $\mathrm{P}_{(\mathrm{i}, \mathrm{j})} \neq \mathrm{p}{ }_{(\mathrm{i}, \mathrm{j})}$ and the embedding error equal zero when $\mathrm{P}_{(\mathrm{i}, \mathrm{j})}=\mathrm{p}_{(\mathrm{i}, \mathrm{j})}$ as shown in Fig. 6 and 7. On the other hand from the analysis result of the embedding errors obtained in the Fig. 6 and 7 . Theoretically, can be calculated the average of embedding errors between the maximum and minimum number in both algorithms. Here the following formula will be useful to calculate the average of embedding errors: thus the embedding error is growth one by one start from $2^{\mathrm{n}-2}+1$ to the maximum embedding error $2^{\mathrm{n}-1}$ and then go down to the $2^{\mathrm{n}-2}+1$ and growth again as shown in Fig.6 and 7. However when the number ' $i$ ' of embed errors are grow up one by one start from $2^{\mathrm{n}-2}+1$ to the maximum embedding error $2^{\mathrm{n}-1}$, thus the formula will be useful to calculate as:

$$
\begin{aligned}
& \sum_{i=1}^{2^{n-2}} i=1+2+\ldots+2^{n-2}=\frac{2^{n-2}\left(2^{n-2}+1\right)}{2}=2^{n-3}\left(2^{n-2}+1\right) \\
& \sum_{i=1}^{2^{n-1}} i=1+2+\ldots+2^{n-1}=\frac{2^{n-1}\left(2^{n-1}+1\right)}{2}=2^{n-2}\left(2^{n-1}+1\right)
\end{aligned}
$$

Subtracting Eq. 3a and $3 b$, then we get the summation of the embedding errors ' $i$ ':

$$
2^{2 \mathrm{n}-3}+2^{\mathrm{n}-2}-2^{2 \mathrm{n}-5}-2^{\mathrm{n}-3}=2^{\mathrm{n}-2}\left(2^{\mathrm{n}-1}-2^{\mathrm{n}-3}+1\right)-2^{\mathrm{n}-3}
$$

From Eq. $3 c$ the average of embedding errors in both algorithms between the cover image and watermarked image can be derived by Eq. $3 \mathrm{~d}$ :

$$
\begin{aligned}
\text { The average of embedding error } & =\frac{2^{\mathrm{n}-2}\left(2^{\mathrm{n}-1}-2^{\mathrm{n}-3}+1\right)-2^{\mathrm{n}-3}}{2^{\mathrm{n}-2}} \\
& =\frac{2\left(2^{\mathrm{n}-1}-2^{\mathrm{n}-3}+1\right)-1}{2}
\end{aligned}
$$

When the $\mathrm{P}_{(\mathrm{i}, \mathrm{j})} \neq \mathrm{p}$ " $(\mathrm{i}, \mathrm{j})$. Suppose that all the pixels in the cover image are used for the embedding of watermark bit by simple (LSB or $\mathrm{MSB}_{6}$ ) substitution method. Theoretically, in the average of worst mean square error in both algorithms are derived from Eq. 3c and $3 \mathrm{~d}$ as:

$$
\operatorname{Averg} \cdot \operatorname{WMSE}^{*}=\frac{1}{\mathrm{M} \times \mathrm{N}} \sum_{\mathrm{i}=1}^{\mathrm{M}} \sum_{\mathrm{j}=1}^{\mathrm{N}}\left[\frac{2\left(2^{\mathrm{n}-1}-2^{\mathrm{n}-3}+1\right)-1}{2}\right]^{2}
$$

Averg. $\operatorname{WMSE}^{*}=\left[\frac{2\left(2^{\mathrm{n}-1}-2^{\mathrm{n}-3}+1\right)-1}{2}\right]^{2}$

From the above Eq. 3f, theoretically, can be derived by Eq. $2 \mathrm{c}$ as well as the maximum of worst mean square error are Max.WMSE ${ }^{*}=\left(2^{\mathrm{n}-1}\right)^{2}$ and the minimum are Min.WMSE ${ }^{*}=\left(2^{\mathrm{n}-2}+1\right)^{2}$ are obtained after applying modified algorithm.

PAP-algorithm-2: We modified the scheme of Chan and Cheng (2004) using Optimal Pixel Adjustment Process (OPAP) the proposed embedding algorithm in the $\mathrm{k}$, means capacity of embedding data bit in LeastSignificant-Bit-n $\left(\mathrm{k}-\mathrm{LSB}_{\mathrm{n}}\right)$ of the cover image, where $\mathrm{k}$ given the high capacity of the embedding data bit. while the embedding data bit in the $\mathrm{LSB}_{1}$ when $\mathrm{k}=1, \mathrm{LSB}_{1,2}$ when $\mathrm{k}=2, \mathrm{LSB}_{1,2,3}$ when $\mathrm{k}=3$ and $\mathrm{LSB}_{1,2,3,4}$ when $\mathrm{k}=4$, where the maximum embedding errors grow up respectively from $\{1,3,7$ and 15$\}$ depending in the value of $k$. Notice that in the directly replacement of embedding data bit the embedding errors grow depending on the $\mathrm{k}$, where the maximum embedding error equal 15 when $\mathrm{k}=4$. So that will be applying the OPAP algorithm in the Most Significant Bit-n $\left(\mathrm{MSB}_{6}\right)$ without using $\mathrm{k}$ of capacity. However the embedding errors in $\mathrm{MSB}_{6}$ equal $=32$ are greater than with compared by using capacity of k-LSB when embedding data bit in $\left(\operatorname{LSB}_{1,2,3,4}\right)$ are equal 15. Let is $\mathrm{P}_{(\mathrm{i}, \mathrm{j})}, \mathrm{P}_{(\mathrm{i}, \mathrm{j})}$ and $\mathrm{P}_{(\mathrm{i}, \mathrm{j})}$ be the corresponding pixel values of a pixel in the cover image, the embedding image $\mathrm{P}_{(\mathrm{i}, \mathrm{j})}$ obtained by the embedding algorithm FOBCB set of Most-SignificantBit-n $\left(\mathrm{MSB}_{6}\right)$ scheme and the refined embedding image obtained after the modified PAP-algorihm2 $\mathrm{P}_{(\mathrm{i}, \mathrm{j})}$. Let absolute $\delta_{(\mathrm{i}, \mathrm{j})}=\left|\mathrm{P}_{(\mathrm{i}, \mathrm{j})}^{\prime}-\mathrm{P}_{(\mathrm{i}, \mathrm{j}, \mathrm{j}}\right|$ be the embedding error between $\mathrm{P}_{(\mathrm{i}, \mathrm{j})}$ and $\mathrm{P}_{(\mathrm{i}, \mathrm{j})}$, therefore, $-2^{\mathrm{n}}<\delta_{(\mathrm{i}, \mathrm{j})}<2^{\mathrm{n}}$, the value of $\delta_{(\mathrm{i}, \mathrm{j})}$ can be further segmented into three intervals, such that: Interval-1: $2^{\mathrm{n}-1}<\delta_{(\mathrm{i}, \mathrm{j})}<2^{\mathrm{n}}$. Interval-2: $-2^{\mathrm{n}-1} \leq$ $\delta_{(\mathrm{i}, \mathrm{j})} \leq 2^{\mathrm{n}-1}$. Interval-3: $-2^{\mathrm{n}}<\delta_{(\mathrm{i}, \mathrm{j})}<-2^{\mathrm{n}-1}$.

The PAP-algorithm-2 based on the three intervals, which modifies $\mathrm{P}_{(\mathrm{i}, \mathrm{j})}$ to form the embedding pixel $\mathrm{P}_{(\mathrm{i}, \mathrm{j})}$, can be described as follows:

Case 1: $\left(2^{\mathrm{n}-1}<\delta_{(\mathrm{i}, \mathrm{j})}<2^{\mathrm{n}}\right)$ : If $\mathrm{P}_{(\mathrm{i}, \mathrm{j},} \geq 2^{\mathrm{n}}$, then $\mathrm{P}_{(\mathrm{i}, \mathrm{j})}{ }^{\prime}=\mathrm{P}_{(\mathrm{i}, \mathrm{j})}^{\prime}-2^{\mathrm{n}}$; otherwise $\mathrm{P}_{(\mathrm{i}, \mathrm{j})}^{\prime \prime}=\mathrm{P}_{(\mathrm{i}, \mathrm{j})}^{\prime}$

Case 2: $\left(-2^{\mathrm{n}-1} \leq \delta_{(\mathrm{i}, \mathrm{j})} \leq 2^{\mathrm{n}-1}\right): \mathrm{P}_{(\mathrm{i}, \mathrm{j})}=\mathrm{P}_{(\mathrm{i}, \mathrm{j})}$

Case 3: $\left(-2^{\mathrm{n}}<\delta_{(\mathrm{i}, \mathrm{j})}<-2^{\mathrm{n}-1}\right)$ : If $\mathrm{P}_{(\mathrm{i}, \mathrm{j})}<256-2^{\mathrm{n}}$, then $\mathrm{P}^{\prime \prime}{ }_{(\mathrm{i}, \mathrm{j})}=$ $\mathrm{P}_{(\mathrm{i}, \mathrm{j})}^{\prime}+2^{\mathrm{n}}$; otherwise $\mathrm{P}_{(\mathrm{i}, \mathrm{j})}^{\prime \prime}=\mathrm{P}_{(\mathrm{i}, \mathrm{j})}^{\prime}$

Where the $\mathrm{P}_{(\mathrm{i}, \mathrm{j})}$ are obtained by the FOBCB set-of$\mathrm{MSB}_{6}$ with applying PAP-algorithm2 and the embedding error occurred between $\mathrm{P}_{(\mathrm{i}, \mathrm{j})}$ and $\mathrm{P}_{(\mathrm{i}, \mathrm{j})}$ computed by $\left.\delta_{(\mathrm{i}, \mathrm{j})}^{\prime}=\mid \mathrm{P}_{(\mathrm{i}, \mathrm{j})}\right) \mathrm{P}_{(\mathrm{i}, \mathrm{j})} \mid$. 
Analysis: Notice that from the optimal pixel adjustment process of the Chi-Kwong-L.M. Cheng scheme, we know that the scheme using ' $\mathrm{k}$ ' capacity of embed watermark bits, where the algorithm minimized the embedding error from $\left(2^{\mathrm{k}-1}\right)$ to $2^{\mathrm{k}-1}$ as shown in Fig. 8 and 9 are sketching the embedding error as a spring shape, our results obtained as the same analysis of the authors. On the other hand from the our analysis results of the embedding errors obtained in the Fig. 8 and 9. Theoretically, can be calculated the number of embedding errors ' $\mathrm{i}$ ' are start from one to $=2^{\mathrm{k}-1}$ with are growth up one by one. Moreover can be calculated the average of embedding errors, the formula will be useful to calculate the summation of embedding errors ' $\mathrm{i}$ ' can be derived by:

$\sum_{i=1}^{2^{k-1}} \mathrm{i}=1+2+\ldots+2^{k-1}=\frac{2^{k-1}\left(2^{k-1}+1\right)}{2}=2^{k-2}\left(2^{k-1}+1\right)$

Hence from the above Eq. $3 \mathrm{~g}$ of the summation of embedding errors ' $i$ '. Thus the average of embedding errors with ' $\mathrm{k}$ ' capacity of embeds watermark bits in kLSB are derived by Eq. $3 \mathrm{~h}$ :

The average of embedding error $=\frac{2^{k-1}\left(2^{k-1}+1\right)}{2^{k-1}}=\frac{2^{k-1}}{2}$

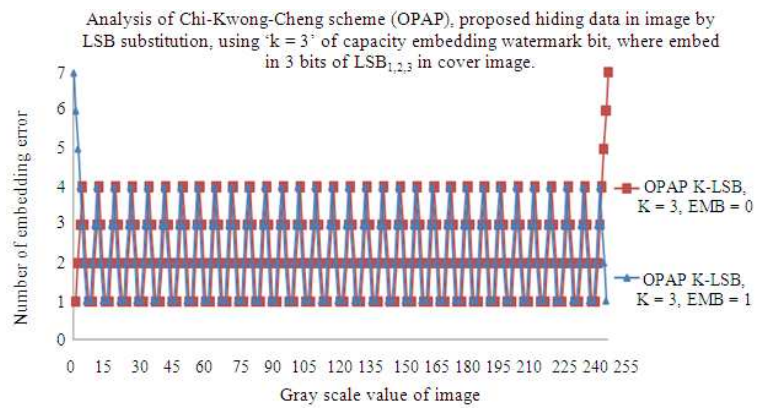

Fig. 8: The embedding error of proposed Chi-Kwong scheme $\mathrm{k}=3$

Analysis of Chi-Kwong-Cheng scheme (OPAP), proposed hiding data in image by LSB substitution, using ' $\mathrm{k}=2^{\prime}$ ' of capacity embedding watermark bit, where embed

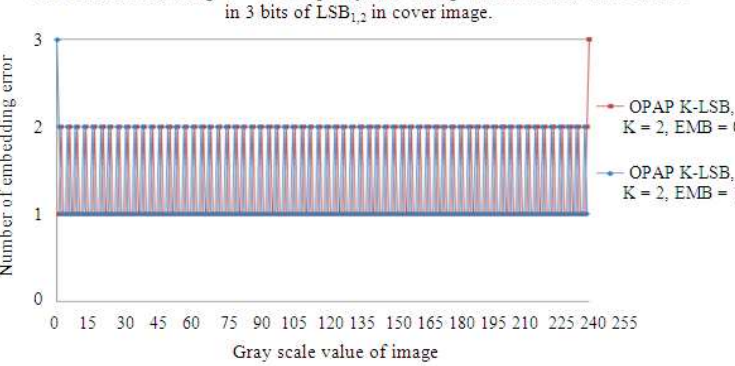

Fig. 9: The embedding error of proposed Chi-Kwong scheme $\mathrm{k}=2$
Thus theoretically from Eq. 2c, the average worst mean square error can be derived as:

$\operatorname{Averg} \cdot \mathrm{WMSE}^{*}=\frac{1}{\mathrm{M} \times \mathrm{N}} \sum_{\mathrm{i}=1}^{\mathrm{M}} \sum_{\mathrm{j}=1}^{\mathrm{N}}\left[\frac{\left(2^{\mathrm{k}-1}+1\right)}{2}\right]^{2}=\left[\frac{\left(2^{\mathrm{k}-1}+1\right)}{2}\right]^{2}$

For example if $\mathrm{P}_{(\mathrm{i}, \mathrm{j})}=255$ and $\mathrm{k}=2$, when the embeds watermark bit equal zero and when $\mathrm{P}_{(\mathrm{i}, \mathrm{j})}=0$, when the embeds watermark bit equal one, thus the embedding error go to the level of max-error in $\operatorname{LSB}_{1,2}=\left(2^{\mathrm{k}}-1\right)=3$. On the other hand if $\mathrm{P}_{(\mathrm{i}, \mathrm{j})}=2,6,10$, $14,18, \ldots . .254$ and $\mathrm{k}=2$, when the embeds watermark bit equal zero and when $\mathrm{P}_{(\mathrm{i}, \mathrm{j})}=1,5,9,13,17, . .253$, when the embedding watermark bit equal one.

Since that our observations, where the embedding error goes to half $(1 / 2)$ of the max-embedding errors added to half $(1 / 2)$, then the max-embed errors of proposed Chi-Kwong-L.M. Cheng scheme are $=\frac{2^{\mathrm{k}}-1+1}{2}=2^{\mathrm{k}-1}$ and the average worst mean square error are obtained by Eq. 3i. On the other hand by using the same algorithm of proposed Chi-Kwong-Cheng scheme, where modified to the algorithm called PAPalgorithm-3 set of $\mathrm{MSB}_{\mathrm{n}}$ where $\mathrm{n}=\mathrm{k}=6$. Notice that here in this modification the embeds watermark bit adjust only embedded one bit only in each pixel of the cover image, thus that the maximum of embedding errors are $=2^{\mathrm{n}-1}=32$ by the direct replacement embedding process of the simple $\mathrm{MSB}_{\mathrm{n}}$ substitution method when $\mathrm{n}=6$. Otherwise by applying the modified PAP-algorithm-3 set of $\mathrm{MSB}_{6}$ the our analysis results in $\mathrm{MSB}_{6}$ as shown in Fig. 10, where the embedding errors are always constant great to the maximum level in all cases of embed watermark bit tested in $\mathrm{LSB}_{1}, \mathrm{LSB}_{2}, \mathrm{LSB}_{3}, \mathrm{LSB}_{4}, \mathrm{MSB}_{5}$ and $\mathrm{MSB}_{6}=2^{\mathrm{n}-1}$ as shown in Fig. 10. Thus theoretically the worst mean square error are constant equal WMSE ${ }^{*}$ $=\left(2^{\mathrm{n}-1}\right)^{2}$, after Appling the modified PAP-algorithm-3 set of $\mathrm{MSB}_{6}$.

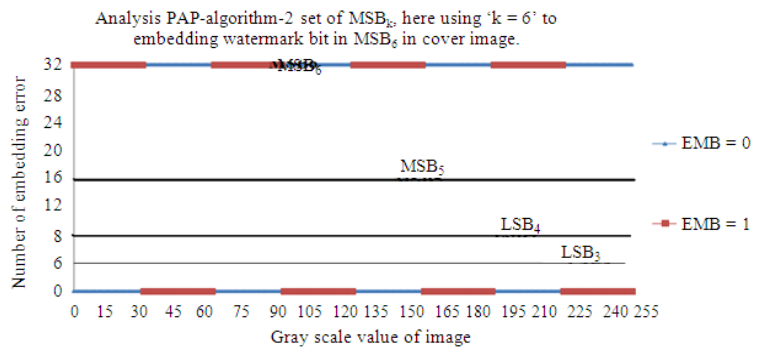

Fig. 10: The embedding error of modified scheme (PAP-algorithm-2) 
PAP-algorithm-3: We modified the algorithm of Aiad and Sada (2007) using Local Pixel Adjustment Process (LPAP) the proposed algorithm used Least-SignificantBit $\left(\mathrm{LSB}_{3}\right)$ to embedded message bit with modified $\mathrm{LSB}_{1,2}$ according to the embedding data bit in $\mathrm{LSB}_{3}$, to minimize the difference between the cover image and the embedding image. So that will be modified the algorithm of LPAP in to Most-Significant-Bit-6 (MSB 6 ) by applying on the embedding algorithm FOBCB set of Most-Significant-Bit-6 (MSB 6 ) scheme (Mehemed et al., 2009), Let's have the cover image $P_{(i, j)}$. Suppose that $\mathrm{MSB}_{6}$ of the cover image is $\mathrm{MSB}_{6}=\left\{\mathrm{MSB}_{1}, \mathrm{MSB}_{2}\right.$, $\left.\mathrm{MSB}_{3}, \ldots, \mathrm{MSB}_{(\mathrm{N} \times \mathrm{M})}\right\}$, where $\mathrm{MSB}_{6}=\{0,1\}$. The embedding process of the watermark bit (EMB) by applying the embedding algorithm FOBCB set of the MSB6 of the cover image to obtain the new embedding image $=\left\{\right.$ newpixel $_{(1,1)}$, newpixel $_{(2,1)}, \ldots$, newpixel $\left._{(\mathrm{N}, \mathrm{M})}\right\}$. To minimize the difference between the old value (pixel) $\mathrm{P}_{(\mathrm{i}, \mathrm{j})}$ in the cover image and the new value (newpixel) in the embedding image, the following embedding algorithm of LPAP set of $\mathrm{MSB}_{6}$ and the size of cover image $(\mathrm{N} \times \mathrm{M})=\mathrm{L}$ :

Step 1: Extract $\mathrm{LSB}_{1}$ set of the cover image, $\mathrm{LSB}_{1}=$ $\left\{\right.$ LSB- $1_{1}$, LSB- $1_{2}, \ldots$, LSB- $\left.1_{\mathrm{L}}\right\}$./first plane

Step 2: Extract $\mathrm{LSB}_{2}$ set of the cover image, $\mathrm{LSB}_{2}=$ $\left\{\right.$ LSB- $2_{1}$, LSB $-2_{2}, \ldots$, LSB $\left.-2_{\mathrm{L}}\right\} . /$ second plane

Step 3: Extract $\mathrm{LSB}_{3}$ set of the cover image, $\mathrm{LSB}_{3}=$ $\left\{\right.$ LSB $-3_{1}$, LSB $-3_{2}, \ldots$, LSB $\left.-3_{\mathrm{L}}\right\} . /$ third plane

Step 4: Extract $\mathrm{LSB}_{4}$ set of the cover image, $\mathrm{LSB}_{4}=$ $\left\{\right.$ LSB $-4_{1}$, LSB $-4_{2}, \ldots$, LSB- $\left.4_{\mathrm{L}}\right\}$./fourth plane

Step 5: Extract $\mathrm{MSB}_{5}$ set of the cover image, $\mathrm{MSB}_{5}=$ $\left\{\right.$ MSB- $5_{1}$, MSB $\left.-5_{2}, \ldots, M S B-5_{L}\right\} . / f i v e$ plane

Step 6: Extract $\mathrm{MSB}_{6}$ set of the cover image, $\mathrm{MSB}_{6}=$ $\left\{\right.$ MSB- $6_{1}$, MSB- $6_{2}, \ldots$, MSB- $\left.6_{\mathrm{L}}\right\} . /$ five plane

Step 7: Set binary watermark image $\mathrm{WL}=\left\{\mathrm{EMB}_{1}\right.$, $\left.\mathrm{EMB}_{2}, \ldots . ., \mathrm{EMB}_{(\mathrm{T} \times \mathrm{U})}\right\}$.

Step 8: For ii $=1$ to $\mathrm{T}$

For $\mathrm{jj}=1$ to $\mathrm{U}$ else

if $\mathrm{MSB}_{6(\mathrm{i}, \mathrm{ij} j)}==\mathrm{EMB}_{(\mathrm{i}, \mathrm{ijj})}$, Then do nothing

if $\mathrm{MSB}_{6(\mathrm{ii}, \mathrm{jj})}==0$ and $\mathrm{EMB}_{(\mathrm{ii}, \mathrm{jj})}==1$, then

$\operatorname{LSB}_{1(\mathrm{ii}, \mathrm{j})}=0 ; \operatorname{LSB}_{2(\mathrm{ii}, \mathrm{j})}=0 ; \operatorname{LSB}_{3(\mathrm{i}, \mathrm{ij})}=0$;

$\mathrm{LSB}_{4(\mathrm{ii}, \mathrm{jj})}=0 ; \mathrm{MSB}_{5(\mathrm{i}, \mathrm{ij})}=0 ; \mathrm{MSB}_{6(\mathrm{ii}, \mathrm{jj})}=\mathrm{EMB}_{(\mathrm{i}, \mathrm{ij})}$; else

if $\mathrm{MSB}_{6(\mathrm{ii}, \mathrm{j} j}==1$ and $\mathrm{EMB}_{(\mathrm{i} \mathrm{i}, \mathrm{jj}}==0$, Then

$\mathrm{LSB}_{1(\mathrm{ii}, \mathrm{jj})}=1 ; \mathrm{LSB}_{2(\mathrm{ii}, \mathrm{j})}=1 ; \mathrm{LSB}_{3(\mathrm{i}, \mathrm{j} j \mathrm{j})}=1$;

$\operatorname{LSB}_{4(\mathrm{i} i \mathrm{jj})}=1 ; \operatorname{MSB}_{5(\mathrm{ii}, \mathrm{j} j)}=1 ; \operatorname{MSB}_{6(\mathrm{ii}, \mathrm{jj})}=\mathrm{EMB}_{(\mathrm{ii}, \mathrm{jj})}$;

\}; \}

$\operatorname{MSB}_{6(\mathrm{ii}, \mathrm{jj})}=\operatorname{EMB}_{(\mathrm{ii}, \mathrm{jj})}$;

\}$;\} ;\}$.

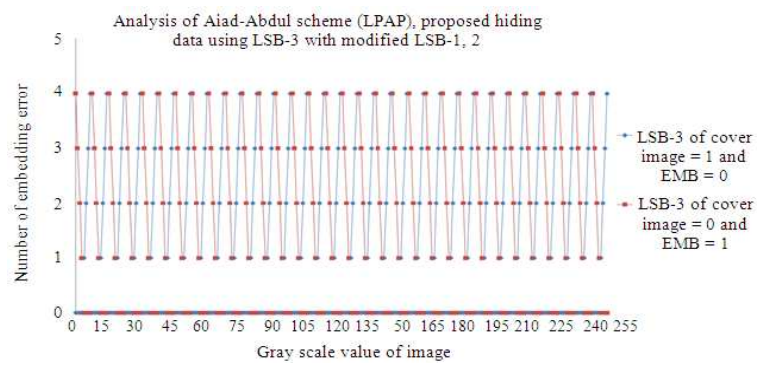

Fig. 11: The embedding error of proposed Aiad and Abdul scheme

Analysis: Notice that from the algorithm LPAP of the Aiad and Abdul scheme, we know that only the first two bits (bits 1-2) as shown in Fig. 5 are modified to improve the image quality. It is obvious that the modification are minimized the embedding errors as shown in Fig. 11 of analysis the possibility gray scale values of image, where are sketching as a spring shape of the embedding error restricted between a minimum ' 1 ' and maximum ' 4 ' of embedding errors.

If $\mathrm{P}_{(\mathrm{i}, \mathrm{j})}=7,15,23,31,39$ and 47 when the embedded watermark bit equal zero and if $\mathrm{P}_{(\mathrm{i}, \mathrm{j})}=0,8$, 16, 24 and 32 as shown in Fig. 11, when the embedded watermark bit equal one, hence that the embedding error grow up to the maximum error in $\mathrm{LSB}_{3}=2^{\mathrm{n}-1}=4$, for example when $\mathrm{P}_{(\mathrm{i}, \mathrm{j})}=(31)_{10}(00011111)_{2}$, then the $\mathrm{LSB}_{3}$ in cover image is ' 1 '. Suppose we need to embed watermark bit is EMB $=0$ in the $\mathrm{LSB}_{3}$ using the Aiad and Abdul scheme, so that the new-pixel will be have $\mathrm{p}^{\prime}=(27)_{10}(00011011)_{2}$, thus the embedding error become $=|31-27|=4$, then the algorithm of Aiad and Abdul scheme, will be set $\mathrm{LSB}_{1,2}$ to ' 1 ' when $\mathrm{LSB}_{3}=1$ and $\mathrm{EMB}=0$. So that the new-pixel $=(27)_{10}$ $(00011011)_{2}$ as the same of our analysis results as shown in Fig. 11. It is obvious that the modification is not decrease the embedding error in this case. Otherwise if the cover image pixel $=(3)_{10}=$ $(00000011)_{2}$. Suppose we need to embed EMB $=1$ in the $\mathrm{LSB}_{3}$, so that the new pixel will be, newpixel = $(00000111)_{2}=(7)_{10}$. Notice that the difference is $|7-3|=$ 4. Then the algorithm will set $\mathrm{LSB}_{1,2}$ to ' 0 ' when EMB $=1$ and $\mathrm{LSB}_{3}$ of cover image $=0$. So that newpixel $=$ $(00000100)_{2}=(4)_{10}$. As you see the deference becomes 4-3 = 1 as the same of analysis results as shown in Fig. 11. On the other hand the same procedures applied on the modification algorithm (PAP-algorithm-3) set of $\mathrm{MSB}_{6}$. Thus our analysis results of the embedding errors in $\mathrm{MSB}_{6}$ as shown in Fig. 12. we see that the embedding errors grow up to the high in $\mathrm{P}_{(\mathrm{i}, \mathrm{j})}=$ 63,191 and 255 as a pyramid shape of embedding error, when the embeds watermark bit equal zero and if $\mathrm{P}_{(\mathrm{i}, \mathrm{j})}=$ 1002 
0, 64, 128 and 192 when the embeds watermark bit equal one, as shown the embedding error greats to the maximum error in $\mathrm{MSB}_{6}=2^{\mathrm{n}-1}=32$ as a pyramid shape of embedding error. On the other hand the embedding errors decreasing to the low level in the $\mathrm{P}_{(\mathrm{i}, \mathrm{j})}=32$, 96,160 and 224 when the embedded watermark bit equal zero and if $\mathrm{P}_{(\mathrm{i}, \mathrm{j})}=31,95,159$ and 223 when the embedded watermark bit equal one, as shown the embedding error go to the minimum error in $\mathrm{MSB}_{6}=1$. Our observation from the analysis result of both algorithms between the $\mathrm{LSB}_{3}$ and $\mathrm{MSB}_{6}$, here the embedding error is grow up one by one start from ' 1 ' to the maximum embedding error $=2^{\mathrm{n}-1}$ and then go down to the ' 1 ' and grow up again, it are sketching as pyramid shape of embedding error, when $\mathrm{P}_{(\mathrm{i}, \mathrm{j})} \neq \mathrm{p}_{(\mathrm{i}, \mathrm{j})}$ and the embedding error equal zero when $\mathrm{P}_{(\mathrm{i}, \mathrm{j})}=\mathrm{p}_{(\mathrm{i}, \mathrm{j})}$ as shown in Fig. 7 and 8 and the maximum embedding error $=2^{\mathrm{n}-1}$ and the minimum embedding error $=1$, where the embed error in the range from $1-2^{\mathrm{n}-1}$ increased one by one. Since that from the analysis result of the embedding error obtained Fig. 11 and 12. Theoretically, can be calculated the average of embedding errors between the maximum and minimum in both algorithms. The following formula will be useful to calculate the average of embedding errors:

$\sum_{i=1}^{2^{n-1}} i=1+2+\ldots+2^{n-1}$

Hence that ' $\mathrm{i}$ ' is the number of embedding errors as Eq. 3 j, then:

$$
\begin{aligned}
& \sum_{i=1}^{2^{n-1}} i=\frac{2^{n-1}\left(2^{n-1}+1\right)}{2} \\
& \sum_{i=1}^{2^{n-1}} i=2^{n-2}\left(2^{n-1}+1\right)
\end{aligned}
$$

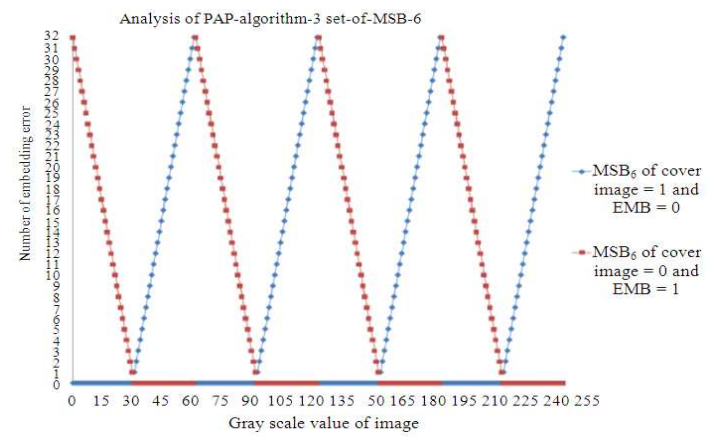

Fig. 12: The embedding error of modified scheme (PAP-algorithm-3)
So that from Eq. 31 the average of embedding errors in both algorithms between the cover image and watermarked image, when the $\mathrm{P}_{(\mathrm{i}, \mathrm{j})} \neq \mathrm{p}_{(\mathrm{i}, \mathrm{j})}$, Theoretically can be derived by:

The average of embedding errors $=\frac{\left(2^{n-1}+1\right)}{2}$

Suppose that all the pixels in the cover image are used for the embedding of watermark bit by simple (LSB or $\mathrm{MSB}_{6}$ ) substitution method, theoretically, from the Eq. $3 \mathrm{~m}$ the average of worst mean square error in both algorithms can be derived by Eq. $2 \mathrm{c}$ as:

Averg. MMSE$^{*}=\frac{1}{M \times N} \sum_{i=1}^{M} \sum_{j=1}^{N}\left[\frac{\left(2^{n-1}+1\right)}{2}\right]^{2}=\left[\frac{\left(2^{n-1}+1\right)}{2}\right]^{2}$

Whereas the maximum and minimum of worst mean square error are equal Max.WMSE ${ }^{*}=\left(2^{n-1}\right)^{2}$ and Min.WMSE ${ }^{*}=1$ after applying both algorithm.

The proposed method: In this letter, have been propose a new novel method of an adaptively pixel adjustment process based on medial pyramid of embedding error set of the Most-Significant Bit-n (APAP-MPOEE $\mathrm{MSBn}_{\text {) }}$ ) is proposed to maybe enhance the gray scale image quality of the watermarked image obtained by a new digital watermarking technique in spatial domain by applying Falling-Off-Boundary In Corners Board (FOBCB) of gray scale images with the random pixel manipulation set of the Most-SignificantBit-6, $\left(\mathrm{MSB}_{\mathrm{n}}\right)$, where $5 \leq \mathrm{n} \leq 8$ as shown in Fig. 5 . The basic concept of the pixel adjustment process of the $\mathrm{LSB}_{\mathrm{n}}$, when $1 \leq \mathrm{n} \leq 4$ based on the technique proposed in (Kevin et al., 2005; Chan and Cheng, 2004; Aiad and Sada, 2007; Wang et al., 2000). Hence that the ideas are derive from the our analyzed of previous methods and modified algorithms as shown in Fig. 6-12 are described in study with analysis and modified the previous methods. However the embedding errors are sketched as a pyramid or spring shape, furthermore the embedding error are restricted between the maximum and minimum of embedding errors. Here in our proposed method we used to embed watermark bits and to trying to minimize the embedding error in to the medial pyramid of embedding error.

Let $\mathrm{P}_{(\mathrm{i}, \mathrm{j})}$, ${ }^{\prime} \mathrm{P}_{(\mathrm{i}, \mathrm{j})}$ and ' $\mathrm{P}_{(\mathrm{i}, \mathrm{j})}$ be the corresponding pixel values of the cover image $F_{(i, j)}$ that contains $(M \times N)$ pixels represent the size of image, ' $\mathrm{P}_{(\mathrm{i}, \mathrm{j})}$ the watermarked image $f_{(i, j)}$ obtained by a new digital watermarking technique in the spatial domain by applying falling-off-boundary in corners board of cover 
image with the random pixel manipulation set of the Most Significant Bit-6 $\left(\mathrm{MSB}_{6}\right)$ and " $\mathrm{P}_{(\mathrm{i}, \mathrm{j})}$ the refined watermarked image obtained after the applying proposed method of an Adaptively Pixel Adjustment Process based on Medial Pyramid Of Embedding Error (APAP-MPOEE) by applying Falling-Off-Boundary in Corners Board (FOBCB) of gray scale images set of the Most-Significant Bit-6 (MSB $)$ with the random pixel manipulation (APAP-MPOEE-FOBCB ${ }_{\mathrm{MSB}}$ ). Let's $\Omega$ ' $=\left|{ }^{\prime} P_{(\mathrm{i}, \mathrm{j})}-\mathrm{P}_{(\mathrm{i}, \mathrm{j})}\right|$ be the embedding error between $\mathrm{P}_{(\mathrm{i}, \mathrm{j})}$ and ' $\mathrm{P}_{(\mathrm{i}, \mathrm{j})}$ according to the embedding process of the falling-off-boundary in corners board of cover image with the random pixel manipulation set of the $\mathrm{MSB}_{6}$ described in described in previous methods, where ${ }^{~} \mathrm{P}_{(\mathrm{i}, \mathrm{j})}$ is obtained by the direct replacement of Embedded Watermark Bit $(E M B) W_{(i, j)}$ equal zero EMB $={ }^{\prime} 0$ ' or equal one $\mathrm{EMB}={ }^{\prime} 1$ ' in the $\mathrm{MSB}_{6}$ of the cover image pixel.

In this study we shall propose a new novel method of APAP-MPOEE set of the MSB 6 by applying fallingoff-boundary in corners board of cover image with the random pixel manipulation blind in spatial domain (APAP-MPOEE-FOBCB ${ }_{\mathrm{MSB}}$ ) maybe to enhance the image quality of the watermarked image to great fidelity and imperceptibility under three steps:

Step 1: Extract pixel from the cover image $\mathrm{P}_{(\mathrm{i}, \mathrm{j})}$ and converted in to the binary bits $\left(\operatorname{LSB}_{(1,2,3,4)}\right.$ and $\left.\operatorname{MSB}_{(5,6,7,8)}\right)$ as shown in Fig. 5, then set of the MostSignificant-Bit-6 $\left(\mathrm{MSB}_{6}\right)$ in each pixel within the boundary of corners board, as well as when the $\mathrm{MSB}_{6}$ equal zero MSB = ' 0 ' and the Embedded Watermark Bit $(E M B)$ of the binary log image $\mathrm{W}(\mathrm{i}, \mathrm{j})$ equal zero $\mathrm{EMB}={ }^{\prime} 0$ ' or when the $\mathrm{MSB}_{6}$ of cover image pixel equal one $\mathrm{MSB}_{6}={ }^{\prime} 1$ ' and when the EMB of the binary log image equal one $\mathrm{EMB}=$ ' 1 ', if $\mathrm{MSB}_{6}=\mathrm{EMB}$, then do nothing, whereas the APAP-MPOEE of watermarked pixel " $\mathrm{P}_{(\mathrm{i}, \mathrm{j})}=$ cover image pixel $\mathrm{P}_{(\mathrm{i}, \mathrm{j})}$. Where the Embedded Bit (EMB) of the binary log image is the same as bit value of $\mathrm{MSB}_{6}$ in cover image. Otherwise when the $\mathrm{MSB}_{6}$ in cover image not equal the Embedded watermark Bit (EMB), $\mathrm{MSB}_{6} \neq \mathrm{EMB}$ thus the pixel value of cover image $\mathrm{P}_{(\mathrm{i}, \mathrm{j})}$ can be further segmented into intervals, whereas the maximum pixel value of cover image in interval with 8 bit at in the range $0 \leq \mathrm{p}_{(\mathrm{i}, \mathrm{j})}<256$, theoretically can be derived the intervals depending on the error value $\left(2^{\mathrm{n}-1}\right)$ in each bit as:

The number of intervals in $\mathrm{MSB}_{\mathrm{n}}=\frac{256}{2^{\mathrm{n}-1}}=8$

From Eq. 4a the all number of intervals in $\mathrm{MSB}_{\mathrm{n}}=8$, when $\mathrm{n}=6$. Furthermore will be divided the eight intervals depending on the step of the embedding Step 2: when $\mathrm{MSB}_{6}$ equal zero $\left(\mathrm{MSB}_{6}={ }^{\prime} 0\right.$ ') and the Embedded Watermark Bit (EMB) equal one $(\mathrm{EMB}=$ '1'). Step 3: when $\mathrm{MSB}_{6}$ equal one $\left(\mathrm{MSB}_{6}={ }^{\prime} 1\right.$ ') and the Embedded watermark Bit (EMB) equal zero (EMB $=$ ' 0 '). From step 2 and 3, theoretically can be further segmented into four intervals in step 2 and 3 derived by:

The number of intervals in each step ${ }_{2,3}=\frac{256}{2^{\mathrm{n}}}=4$

Hence that the embedding process in the $\mathrm{MSB}_{6}$ of the cover image pixel in the boundary of corners board to form the watermarked pixel " $\mathrm{P}_{(\mathrm{i}, \mathrm{j})}$ that required eight intervals as Eq. 4a, thus each interval will be divided in to two intervals to minimizing the embedding error in to the medial pyramid of embedding error, then will be get sixteen intervals, can be described as: First: Will be divided each interval in to two intervals as shown in Fig. 13 and 14. Second: Added $\left(2^{n-2}\right)$ in each start interval to get the end of a new interval as shown in Fig. 13 and 14, where are from the interval-1 will be get two intervals as shown in Fig. 13 and in case.1. Hence that each interval from (1-8) is divided in to the half (1/2) in each interval, to obtained sixteen intervals as in case.(1-8) in step: (2 and 3) shown in Fig. 13 and 14, can be derived in step: (2 and 3). Notice that $n$ in $\mathrm{MSB}_{\mathrm{n}}$ within interval $6 \leq n \leq 8$ :

Step 2: In this step when the $\mathrm{MSB}_{6}=$ ' 0 ' in the pixel of cover image and $\mathrm{EMB}=$ ' 1 ' $\mathrm{o} \mathrm{f}$ the embedded watermark bit, then the value pixels of cover image $\mathrm{P}_{(\mathrm{i}, \mathrm{j})}$ can be further segmented into four intervals as Eq. $4 \mathrm{~b}$ from the total intervals as Eq. $4 \mathrm{a}$, such that:

Interval 1: $0 \leq \mathrm{p}_{(\mathrm{i}, \mathrm{j})}<2^{\mathrm{n}-1}$

Interval 2: $2^{\mathrm{n}} \leq \mathrm{p}_{(\mathrm{i}, \mathrm{j})}<3 \times 2^{\mathrm{n}-1}$

Interval 3: $2^{\mathrm{n}+1} \leq \mathrm{p}_{(\mathrm{i}, \mathrm{j})}<5 \times 2^{\mathrm{n}-1}$

Interval 4: $3 \times 2^{\mathrm{n}} \leq \mathrm{p}_{(\mathrm{i}, \mathrm{j})}<7 \times 2^{\mathrm{n}-1}$

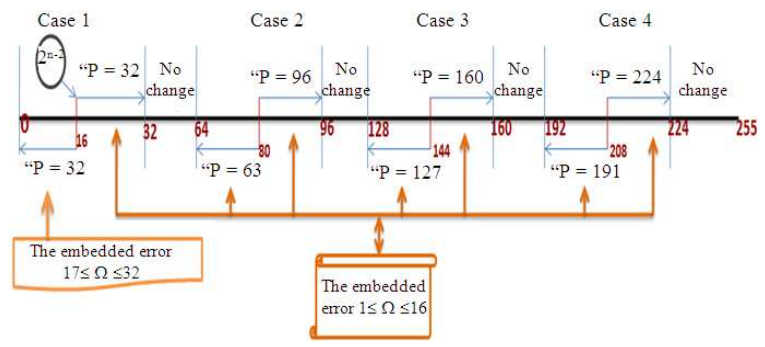

Fig. 13: Interval of embedding process when $\mathrm{MSB}_{6}=0$ and $\mathrm{EMB}=1$ 
Am. J. Applied Sci., 7 (7): 987-1022, 2010

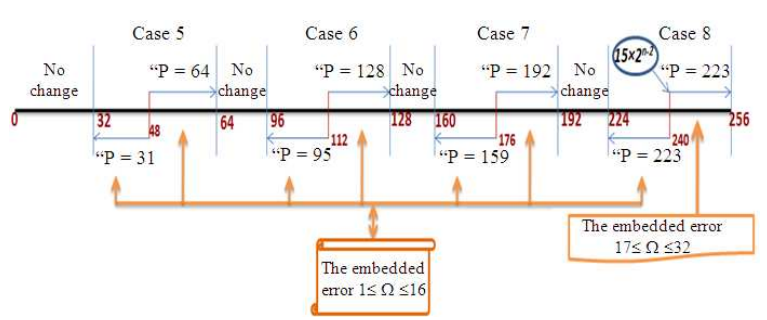

Fig. 14: Interval of embedding process when $\mathrm{MSB}_{6}=1$ and $\mathrm{EMB}=0$

In this step based on four intervals from (1-4), the APAP-MPOEE, which the algorithm requires a checking between the $\mathrm{MSB}_{6}=$ ' 0 ' in the pixel of cover image and $\mathrm{EMB}=$ ' 1 ' of the embedded watermark bit before embedding the watermark bit depending on the nearest of adaptively pixel in the medial pyramid of embedding error to inform the watermarked image " $\mathrm{P}_{(\mathrm{i}, \mathrm{j})}$ as shown in Fig. 13, can be described as follows:

Case 1: $\left(0 \leq \mathrm{P}_{(\mathrm{i}, \mathrm{j})}<2^{\mathrm{n}-1)}\right.$, then

$$
\begin{aligned}
& \text { if }\left(0 \stackrel{(\mathrm{i}, \mathrm{j})}{\leq} \mathrm{P}_{(\mathrm{i}, \mathrm{j})}<2^{\mathrm{n}-2}\right) \text {, then } \\
& \text { "P } \mathrm{P}_{(\mathrm{i}, \mathrm{j})}=2^{\mathrm{n}-1} ; \\
& \text { else } \\
& \text { " } \mathrm{P}_{(\mathrm{i}, \mathrm{j})}=2^{\mathrm{n}-1} ;
\end{aligned}
$$

Case 2: $\left(2^{\mathrm{n}} \leq \mathrm{P}_{(\mathrm{i}, \mathrm{j})}<3 \times 2^{\mathrm{n}-1)}\right.$, then

$$
\text { if }\left(2^{\mathrm{n}} \leq \mathrm{P}_{(\mathrm{i}, \mathrm{j})}<5 \times 2^{\mathrm{n}-2}\right) \text {, then }
$$

else

$$
" P_{(i, j)}=2^{n}-1 \text {; }
$$

end

$$
\text { " } P_{(i, j)}=3 \times 2^{n-1} ;
$$

Case 3: $\left(2^{\mathrm{n}+1} \leq \mathrm{P}_{(\mathrm{i}, \mathrm{j})}<5 \times 2^{\mathrm{n}-1)}\right.$, then

$$
\begin{gathered}
\text { if }\left(2^{\mathrm{n}+1} \leq \mathrm{P}_{(\mathrm{i}, \mathrm{j},}<9 \times 2^{\mathrm{n}-2)}\right. \text {, then } \\
\mathrm{P}_{(\mathrm{i}, \mathrm{j})}=2^{\mathrm{n}+1}-1 ;
\end{gathered}
$$

else

end

$$
\text { " } P_{(i, j)}=5 \times 2^{n-1} \text {; }
$$

Case 4: $\left(3 \times 2^{\mathrm{n}} \leq \mathrm{P}_{\mathrm{(i,j},}<7 \times 2^{\mathrm{n}-1)}\right.$, then

$$
\begin{aligned}
& \text { if }\left(3 \times 2^{\mathrm{n}} \stackrel{\leq}{\leq} \mathrm{P}_{(\mathrm{i}, \mathrm{j})}<13 \times 2^{\mathrm{n}-2)}\right. \text {, then } \\
& \text { " } \mathrm{P}_{(\mathrm{i}, \mathrm{j})}=3 \times 2^{\mathrm{n}}-1 ; \\
& \text { else } \\
& \text { " } \mathrm{P}_{(\mathrm{i}, \mathrm{j})}=7 \times 2^{\mathrm{n}-1} ; \\
& \text { End }
\end{aligned}
$$

Step 3: In this step when the $\mathrm{MSB}_{6}={ }^{\prime} 1$ ' in the pixel of cover image and $\mathrm{EMB}={ }^{\prime} 0$ ' o $\mathrm{f}$ the embedded watermark bit, then the value pixels of cover image $P_{(i, j)}$ can be further segmented into four intervals as Eq. $4 \mathrm{~b}$ from the total intervals as Eq. $4 \mathrm{a}$, such that:

Interval 5: $2^{\mathrm{n}-1} \leq \mathrm{p}_{(\mathrm{i}, \mathrm{j})}<2^{\mathrm{n}}$

Interval 6: $3 \times 2^{n-1} \leq \mathrm{p}_{(\mathrm{i}, \mathrm{j})}<2^{\mathrm{n}+1}$
Interval 7: $5 \times 2^{\mathrm{n}-1} \leq \mathrm{p}_{(\mathrm{i}, \mathrm{j})}<3 \times 2^{\mathrm{n}}$

Interval 8: $7 \times 2^{\mathrm{n}-1} \leq \mathrm{p}_{(\mathrm{i}, \mathrm{j})}<2^{\mathrm{n}+2}$

In this step based on four intervals from (5-8), the APAP-MPOEE, which the algorithm requires a checking between the $\mathrm{MSB}_{6}=$ ' 1 ' in the pixel of cover image and $\mathrm{EMB}=$ ' 0 ' of the embedded watermark bit before embedding the watermark bit depending on the nearest of adaptively pixel in the medial pyramid of embedding error to inform the watermarked image " $\mathrm{P}_{(\mathrm{i}, \mathrm{j})}$ as shown in Fig. 14, can be described as follows:

Case 5: $\left(2^{\mathrm{n}-1} \leq \mathrm{P}_{(\mathrm{i}, \mathrm{j})}<2^{\mathrm{n})}\right.$, then

$$
\begin{gathered}
\text { if }\left(2^{n-1} \leq P_{(i, j)}<3 \times 2^{n-2)}\right. \text {, then } \\
\text { " } P_{(i, j)}=2^{n-1}-1 ;
\end{gathered}
$$

else

if $(6 \leq \mathrm{n} \leq 7)$, then

$$
\text { " } P_{(i, j)}=2^{n} \text {; }
$$

else

$$
\text { " } P_{(i, j)}=2^{n-1}-1 ;
$$

end

Case 6: $\left(3 \times 2^{\mathrm{n}-1} \leq \mathrm{P}_{(\mathrm{i}, \mathrm{j})}<2^{\mathrm{n}+1)}\right.$, then

$$
\begin{gathered}
\text { if }\left(3 \times 2^{\mathrm{n}-1} \stackrel{(\mathrm{i}, \mathrm{j})}{\leq} \mathrm{P}_{(\mathrm{i}, \mathrm{j})}<7 \times 2^{\mathrm{n}-2)},\right. \text { then } \\
\mathrm{P}_{(\mathrm{i}, \mathrm{j})}=3 \times 2^{\mathrm{n}-1}-1 ;
\end{gathered}
$$

else

$$
\begin{aligned}
& \text { if }(\mathrm{n}==6) \text {, then } \\
& \quad " \mathrm{P}_{(\mathrm{i}, \mathrm{j})}=2^{\mathrm{n}+1} ; \\
& \text { else " } \mathrm{P}_{(\mathrm{i}, \mathrm{j})}=3 \times 2^{\mathrm{n}-1}-1 ; \\
& \text { end }
\end{aligned}
$$

end

Case 7: $\left(5 \times 2^{\mathrm{n}-1} \leq \mathrm{P}_{(\mathrm{i}, \mathrm{j})}<3 \times 2^{\mathrm{n})}\right.$, then

if $\left(5 \times 2^{n-1} \leq \mathrm{P}_{(\mathrm{i}, \mathrm{j})}<11 \times 2^{\mathrm{n}-2}\right)$, then

else

$$
\text { " } \mathrm{P}_{(\mathrm{i}, \mathrm{j})}=5 \times 2^{\mathrm{n}-1}-1 \text {; }
$$

end

$$
\text { " } P_{(i, j)}=3 \times 2^{n} ;
$$

Case 8: $\left(7 \times 2^{\mathrm{n}-1} \leq \mathrm{P}_{(\mathrm{i}, \mathrm{j})}<2^{\mathrm{n}+2)}\right.$, then

$$
\begin{aligned}
& \text { if }\left(7 \times 2^{\mathrm{n}-1} \leq \mathrm{P}_{(\mathrm{i}, \mathrm{j})}<15 \times 2^{\mathrm{n}-2)}\right. \text {, then } \\
& \quad \text { " } \mathrm{P}_{(\mathrm{i}, \mathrm{j})}=7 \times 2^{\mathrm{n}-1}-1 ; \\
& \text { else } \\
& \text { " } \mathrm{P}_{(\mathrm{i}, \mathrm{j})}=7 \times 2^{\mathrm{n}-1}-1 ; \\
& \text { End }
\end{aligned}
$$

Encoding process of APAP-MPOEE: The proposed algorithm of APAP-MPOEE-MSBn developed to the most-significant-bit ${ }_{n} \mathrm{MSB}_{\mathrm{n}}$ in spatial domain, whereas $5<\mathrm{n} \leq 8$. Moreover the proposed algorithm of APAPMPOEE-MSBn used for embedding watermark bits in the cover image and before embedding requires a checking between the $\mathrm{MSB}_{6}$ in the pixel of cover image and EMB of the embedded watermark bit depending on the 
Am. J. Applied Sci., 7 (7): 987-1022, 2010

nearest of the adaptively pixel in the medial pyramid of embedding error to inform the watermarked image " $\mathrm{P}_{(\mathrm{i}, \mathrm{j})}$. First: Have been permute the pixel of watermark image before inserted to protect the watermark bit by rearranged pixel according to the security key, to avoid possible attack as we used in the published paper (Mehemed et al., 2009) reviewed in previous methods within step.1 within step.1. Second: After permute the pixel of watermark image, then will be insertion with redundantly distributed the watermark bits over many pixels of the cover image, using a small watermark image $16 \times 16$, are added simultaneously to improve the capacity and to ensure robustness. Third: The following algorithm of APAP-MPOEE set of the $\mathrm{MSB}_{6}$ :

$\mathrm{P}=$ imread(num $2 \operatorname{str}($ Cover gray scale image) $)$; then extracted pixel from the cover image, $F=\left\{\right.$ pixel $_{1}$, $\left.\operatorname{pixel}_{2}, \ldots, \operatorname{pixel}_{(\mathrm{M} \times \mathrm{N})}\right\}=\mathrm{P}_{(\mathrm{i}, \mathrm{j})}$ and convert the cover image pixel $\mathrm{P}_{(\mathrm{i}, \mathrm{j})}$ to the binary bits (8 bit grey values) as a $\operatorname{LSB}_{(1,2,3,4)}$ and $\operatorname{MSB}_{(5,6,7,8)}$ from right to left as shown in Fig. 5, then set of the Most-Significant Bit-n $\left(\mathrm{MSB}_{6}\right)$ in each pixel of the cover image $\mathrm{P}_{(\mathrm{i}, \mathrm{j})}$. Let's have a binary watermark image $\mathrm{WL}_{(\mathrm{i}, \mathrm{j}}$, whereas the size of $\mathrm{WL}=[\mathrm{T}, \mathrm{U}]$ and $\mathrm{WL}=\{0,1\}, \mathrm{T}$ equal $\mathrm{U}$, then extracted binary bits from the watermark image as $\mathrm{EMB}=\left\{\mathrm{EMB}_{1}, \mathrm{EMB}_{2}, \ldots, \mathrm{EMB}_{(\mathrm{T} \times \mathrm{U})}\right\}=\mathrm{EMB}_{(\mathrm{i}, \mathrm{j})}$, where is $n=6$; then can be described as follows:

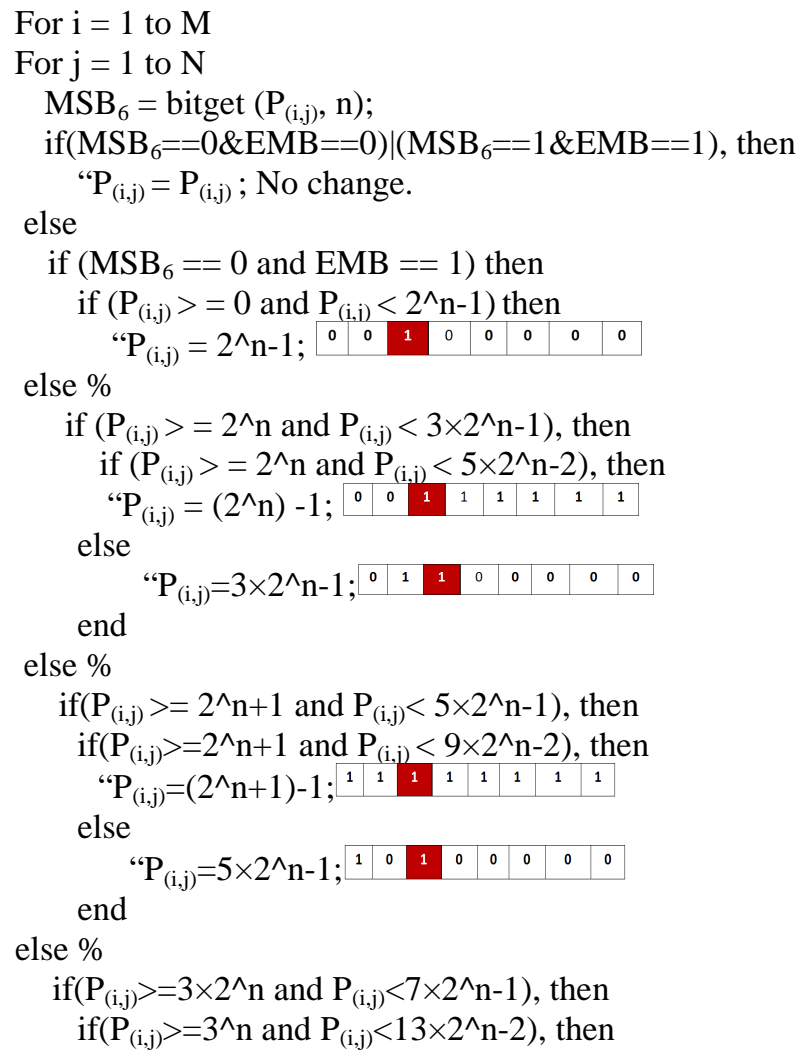

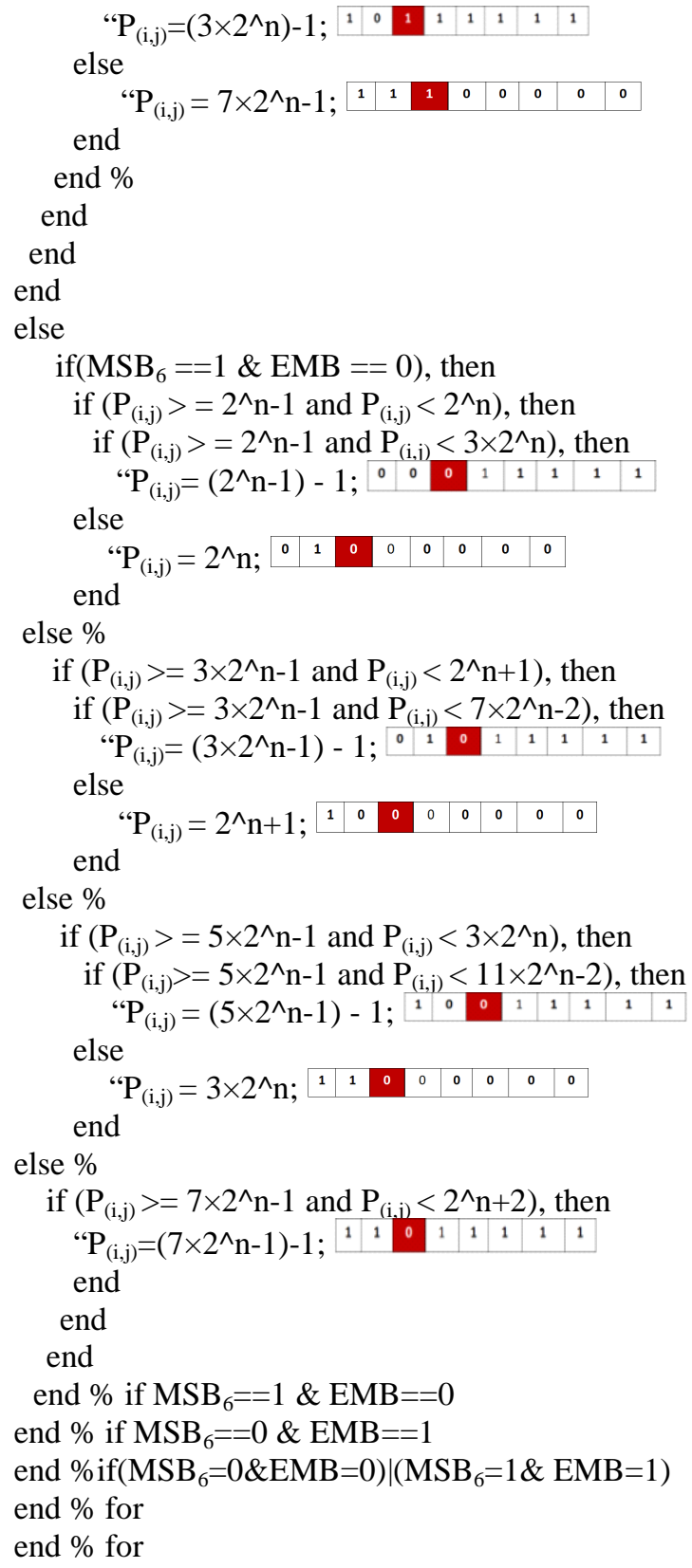

Four: From the above algorithm of proposed method (APAP-MPOEE set of the $\mathrm{MSB}_{6}$ ) used by applying a falling-off-boundary in corners board of the cover image with the random pixel manipulation in the spatial domain (APAP-MPOEE-FOBCB ${ }_{M S B}$ ). The

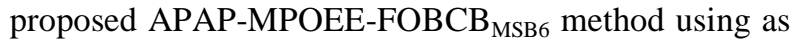
a embeds watermark bits in a boundary in corners board of the cover image and before embedding requires a checking between the $\mathrm{MSB}_{6}$ in the boundary in corners board pixel of the cover image and EMB of the embedded watermark bit, depending on the nearest of 
the adaptively pixel in the medial pyramid of embedding error to inform the watermarked image " $\mathrm{P}_{(\mathrm{i}, \mathrm{j})}$ obtained by a APAP-MPOEE-FOBCB ${ }_{\mathrm{MSB} 6}$ scheme as shown in the flowchart for the embedding process of the APAP-MPOEE-FOBCB ${ }_{\mathrm{MSB} 6}$ scheme in Fig. 15.

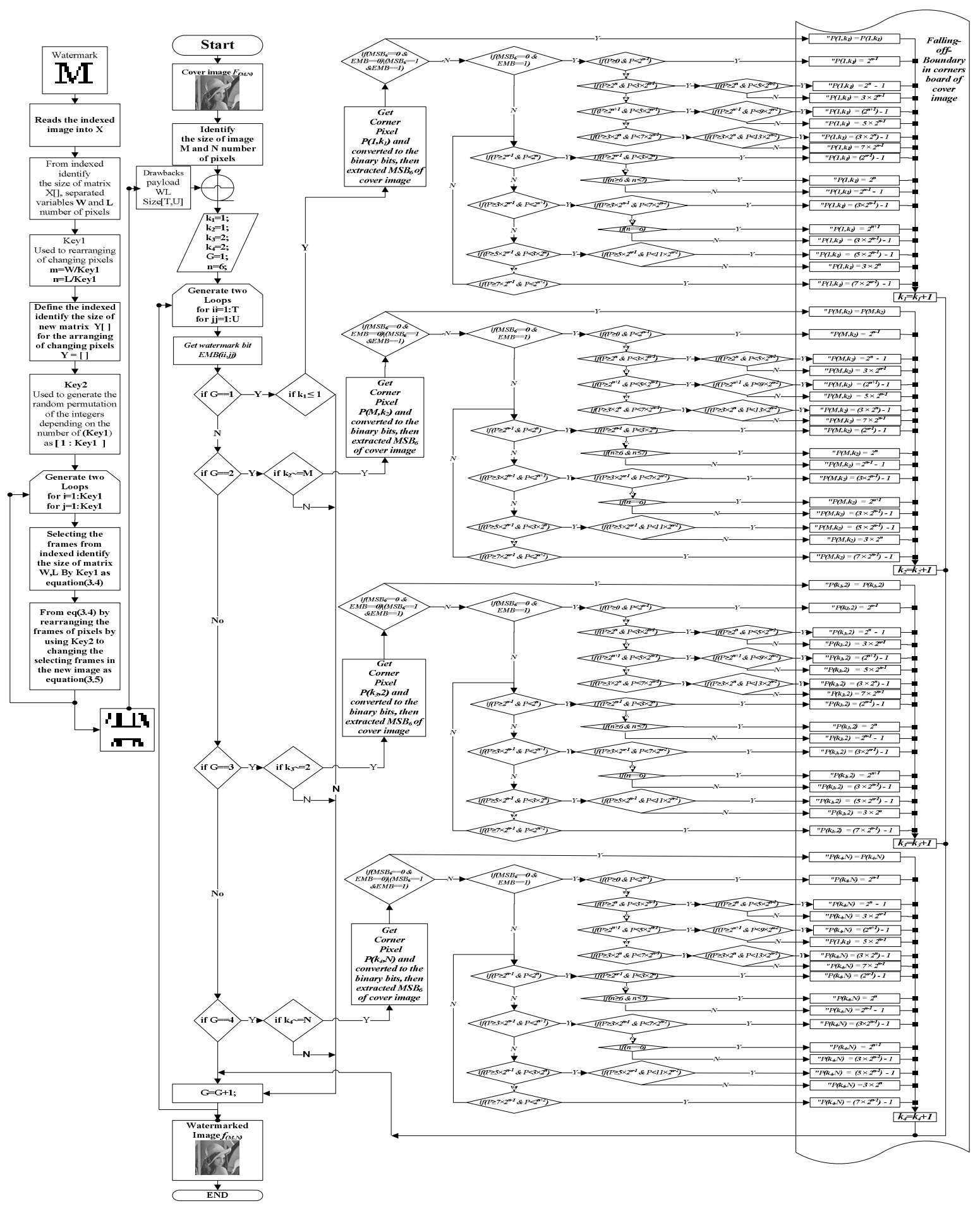

Fig. 15: The flowchart embedding process of proposed method (APAP-MPOEE-FOBCB ${ }_{\mathrm{MSB} 6}$ ) 1007 


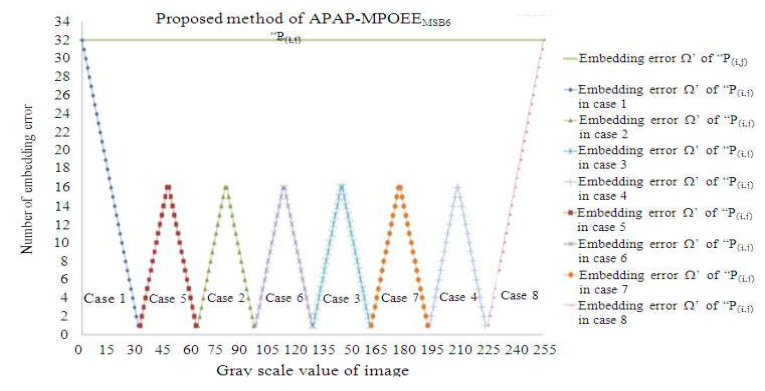

Fig. 16: Comparisons proposed method of APAP${ }_{\text {MPOEE }}{ }_{\text {MSB6 }}$ with the ${ }^{~} \mathrm{P}_{(\mathrm{i}, \mathrm{j})}$ are obtained by the direct replacement of embeds watermark bits.

Decoding process of APAP-MPOEE: A decoder using to extracted watermark bits from drawbacks in falling-off-boundary in corners board of the watermarked image $f_{(M, N)}$ by using inverse the same procedure of the embedded algorithm without using the steps of embedding process in proposed method adjust recovery the watermark bits from the falling-offboundary in corners board in watermarked image depending on the sequence number $G$ to know the manipulation pixel between boundary corners board in the watermarked image $f_{(\mathrm{M}, \mathrm{N})}$ and then select one of drawbacks in the $\mathrm{MSB}_{6}$, after extracted watermark required a secret $\mathrm{Key}_{1,2}$ to rearranging the change of the frames per row, then the watermark in original form is thus obtained. This is completes watermark extraction process. A quantitative estimation for the quality of extracted watermark image $\mathrm{W}_{(\mathrm{i}, \mathrm{j})}$ under inspection with or without external attacks by compared with the original watermark $\mathrm{W}(\mathrm{i}, \mathrm{j})$ as reference can be expressed as a normalized cross correlation, psnr and similarity function.

Analysis the scheme of APAP-MPOEE ${ }_{\text {MSB6: To }}$ analysis the above algorithm of APAP-MPOEE ${ }_{\mathrm{MSB}}$ according to the cases from (1-8) are generated a sixteen intervals, where are two interval in each pyramid case of embedding error as shown in Fig. 16. First: Let's have the following pixel in the cover image, $\mathrm{P}_{(\mathrm{i}, \mathrm{j})}=(31)_{10}=(00011111)_{2}$. Suppose the embed binary watermark bit equal one $\mathrm{EMB}=1$ in the $\mathrm{MSB}_{6}$, where the $\mathrm{MSB}_{6}$ of the pixel in the cover image $\mathrm{MSB}_{6}$ equal zero $\mathrm{MSB}_{6}=0$, so the ${ }^{\prime} \mathrm{P}_{(\mathrm{i}, \mathrm{j})}$ is obtained by the direct replacement of Embedded watermark Bit (EMB) of binary image $\mathrm{W}(\mathrm{i}, \mathrm{j})$, when $\mathrm{MSB}_{6}={ }^{\prime} 0$ ' and $\mathrm{EMB}={ }^{\prime} 1$ ' in the Most-Significant-Bit-6 $\left(\mathrm{MSB}_{6}\right)$ of the cover image pixel to produce watermarked pixel ${ }^{\prime} \mathrm{P}_{(\mathrm{i}, \mathrm{j})}=$ $(00111111)_{2}=(63)_{10}$. Notice that the difference is $\Omega^{\prime}=$ $\left|' \mathrm{P}_{(\mathrm{i}, \mathrm{j})}-\mathrm{P}_{(\mathrm{i}, \mathrm{j})}\right|$ be the embedding error between $\mathrm{P}_{(\mathrm{i}, \mathrm{j})}$ and ${ }^{\prime} \mathrm{P}_{(\mathrm{i}, \mathrm{j})}$ according to the embedding process of the binary watermark bit equal one EMB $=1$ in the $\mathrm{MSB}_{6}=\mid 63$ $31 \mid=32$, otherwise $\mathrm{P}_{(\mathrm{i}, \mathrm{j})}=(0)_{10}=(00000000)_{2}$. Suppose the embed equal one $\mathrm{EMB}=1$ in the $\mathrm{MSB}_{6}$, where the $\mathrm{MSB}_{6}$ of the pixel in the cover image $\mathrm{MSB}_{6}$ equal zero $\mathrm{MSB}_{6}=0$, so the ${ }^{\prime} \mathrm{P}_{(\mathrm{i}, \mathrm{j})}=(00100000)_{2}=(32)_{10}$. Notice that the difference error is $\Omega^{\prime}=\left|\mathrm{P}_{(\mathrm{i}, \mathrm{j})}-\mathrm{P}_{(\mathrm{i}, \mathrm{j})}\right|=32-0=32$, then the max-embedding error $=32$. Otherwise let's have the following pixel in the cover image, $\mathrm{P}_{(\mathrm{i}, \mathrm{j})}=$ $(32)_{10}=(00010000)_{2}$. Suppose the embed binary watermark bit equal zero EMB $=0$ in the $\mathrm{MSB}_{6}$, where the $\mathrm{MSB}_{6}$ of the pixel in the cover image $\mathrm{MSB}_{6}$ equal one $\mathrm{MSB}_{6}=1$, then the ${ }^{\prime} \mathrm{P}_{(\mathrm{i}, \mathrm{j})}$ is obtained by the direct replacement of Embedded Watermark Bit (EMB) when $\mathrm{MSB}_{6}=$ ' 1 ' and EMB $={ }^{\prime} 0$ ' in the $\mathrm{MSB}_{6}$ of the cover image pixel to produce watermarked pixel ' $\mathrm{P}_{(\mathrm{i}, \mathrm{j})}=$ $(00000000)_{2}=(0)_{10}$. Notice that the difference is $\Omega^{\prime}=$ $\mid$ ' $\left.\mathrm{P}_{(\mathrm{i}, \mathrm{j})}\right) \mathrm{P}_{(\mathrm{i}, \mathrm{j})} \mid$ be the embedding error between $\mathrm{P}_{(\mathrm{i}, \mathrm{j})}$ and ${ }^{\prime} \mathrm{P}_{(\mathrm{i}, \mathrm{j})}$ according to the embedding process of the binary watermark bit equal zero in the $\mathrm{MSB}_{6}=|0-32|=32$, also when $\mathrm{P}_{(\mathrm{i}, \mathrm{j})}=(63)_{10}=(00111111)_{2}$. Suppose the embed equal zero EMB $=0$ in the $\mathrm{MSB}_{6}$, where the $\mathrm{MSB}_{6}$ of the pixel in the cover image $\mathrm{MSB}_{6}$ equal one $\mathrm{MSB}_{6}=1$, then the ${ }^{\prime} \mathrm{P}_{(\mathrm{i}, \mathrm{j})}=(00011111)_{2}=(31)_{10}$. Notice that the difference error is $\Omega^{\prime}=\left|\mathrm{P}_{(\mathrm{i}, \mathrm{j})}-\mathrm{P}_{(\mathrm{i}, \mathrm{j}, \mathrm{j}}\right|=|31-63|=32$, then the max-embedding error $=32$ for all the embedding process, only the embedding error equal zero $\Omega^{\prime}=$ $\left|\mathrm{P}_{(\mathrm{i}, \mathrm{j})}-\mathrm{P}_{(\mathrm{i}, \mathrm{j}, \mathrm{j}}\right|=0$ when the embed watermark bit equal the same of the $\mathrm{MSB}_{6}$ in the cover image pixel. Hence that the embedding errors are constant $\Omega^{\prime}=32$.

Second: From the above analysis have been proposed a novel algorithm of APAP-MPOEE developed to the Most-Significant-bit $\mathrm{MSB}_{\mathrm{n}}$ in spatial domain, where is $5<\mathrm{n} \leq 8$, for trying to enhance the image quality of the watermarked image. Hence that when applying proposed method of APAP-MPOEE set of the $\mathrm{MSB}_{6}$ in spatial domain. For example let's have the following pixel in the cover image, $\mathrm{P}_{(\mathrm{i}, \mathrm{j})}=(31)_{10}=$ $(00011111)_{2}$. Suppose the embed watermark bit equal one $\mathrm{EMB}=1$ in the $\mathrm{MSB}_{6}$, where the $\mathrm{MSB}_{6}$ of the cover image pixel equal zero $\mathrm{MSB}_{6}=0$, then have been apply case. 1 in the proposed method (APAPMPOEE $\left._{\text {MSB }}\right)=" \mathrm{P}_{(\mathrm{i}, \mathrm{j})}=2^{\mathrm{n}-1}=(00100000)_{2}=(32)_{10}$ as shown in Fig. 13. Notice that the difference error is $\Omega=$ $\left|" \mathrm{P}_{(\mathrm{i}, \mathrm{j})}{ }^{-} \mathrm{P}_{(\mathrm{i}, \mathrm{j})}\right|$ be the embedding error between $\mathrm{P}_{(\mathrm{i}, \mathrm{j})}$ and " $\mathrm{P}_{(\mathrm{i}, \mathrm{j})}$ according to the embedding process of the watermark bit equal one EMB $=1$ in the $\mathrm{MSB}_{6}=\mid 32-$ $31 \mid=1$. Another example let's have $\mathrm{P}_{(\mathrm{i}, \mathrm{j})}=(16)_{10}=$ $(00010000)_{2}$. Suppose the embed equal one in the $\mathrm{MSB}_{6}$, where the $\mathrm{MSB}_{6}$ of the cover image pixel equal zero, then have been apply case. 1 in the proposed method (APAP-MPOEE $\left.{ }_{\text {MSB } 6}\right)={ }^{\prime} \mathrm{P}_{(\mathrm{i}, \mathrm{j})}=2^{\mathrm{n}-1}=$ $(00100000)_{2}=(32)_{10}$ is obtained by the direct replacement of APAP-MPOEE ${ }_{\text {MSB6 }}$ as shown in Fig. 13, 
so that the difference error is $\Omega=\mid$ ' $\mathrm{P}_{(\mathrm{i}, \mathrm{j})}-\mathrm{P}_{(\mathrm{i}, \mathrm{j},}|=| 32-16 \mid$ $=16$, notices that the embedded error in the case 1 are become in proposed method as in to a interval $2^{\mathrm{n}-2}$ $\leq \mathrm{P}_{(\mathrm{i}, \mathrm{j})}<2^{\mathrm{n}-1}$, where are in the range from $1 \leq \Omega \leq 16$. It has the same embedding errors in case. 2, 3, 4, 5, 6 and 7 as shown in the intervals of embedding process in Fig. 13 and 14 , where are the embedding errors are minimized to the half $(1 / 2)$ with compared by the direct replacement of the embedded watermark bit. Finally, from the above analysis of proposed method of APAP-MPOEE ${ }_{\mathrm{MSB}}$ according to the cases from (1-8) the embedding errors $\Omega$ increased one by one according to the values of cover image pixel $\mathrm{P}_{(\mathrm{i}, \mathrm{j})}$ in the range from $2^{\mathrm{n}-2} \leq \mathrm{P}_{(\mathrm{i}, \mathrm{j})}<2^{\mathrm{n}-1}$, where are become when $n=6$ in the range of $1 \leq \Omega \leq 16$ as shown in Fig. 16, where is each case minimizing the embedding errors to the medial pyramid of embedding error to inform the watermarked pixel? By the way shown in the sketched of the Fig. 16, each case sketched the pyramid of embedding error are minimized to the half $\left(2^{\mathrm{n}-2}\right)$ of maximum embedding error $\left(2^{\mathrm{n}-1}\right)$, when the values of gray scale in cover image pixel $\mathrm{P}_{(\mathrm{i}, \mathrm{j})}$ in the interval between $2^{\mathrm{n}-2} \leq \mathrm{P}_{(\mathrm{i}, \mathrm{j})}$ $\leq 15 \times 2^{\mathrm{n}-2}$ as shown in Fig. 13 and 14. Otherwise the embedding error $\Omega$ are grow one by one according to the values of gray scale in cover image pixel $\mathrm{P}_{(\mathrm{i}, \mathrm{j})}$ in the range from $17 \leq \Omega \leq 32$ as shown in the first half of case. 1 and in the last half in case. 8 , when the values of gray scale in the cover image pixel $\mathrm{P}_{(\mathrm{i}, \mathrm{j})}$ in the intervals from $0 \leq \mathrm{P}_{(\mathrm{i}, \mathrm{j})}<2^{\mathrm{n}-2}$ as shown in Fig. 13 with case 1 and from $15 \times 2^{n-2}<\mathrm{P}_{(\mathrm{i}, \mathrm{j})}<256$ as shown in Fig. 14 with case 8 . Notices that in the proposed method the embedding errors $\Omega$ are minimized to the half $(1 / 2)$, where are the embedding errors decreased one by one as shown in Fig. 16 in each case, when the values of gray scale image pixel $\mathrm{P}_{(\mathrm{i}, \mathrm{j})}$ in the interval between $2^{\mathrm{n}-2} \leq \mathrm{P}_{(\mathrm{i}, \mathrm{j})} \leq 15 \times 2^{\mathrm{n}-2}$ with compared by the direct replacement of embedding watermark bits ' $\mathrm{P}_{(\mathrm{i}, \mathrm{j})}$ as shown the embedding errors $\Omega$ ' are constant $\Omega^{\prime}=32$. On the other hand from the Fig. 16, theoretically can be calculated the summation of embedding errors in all intervals, but will be neglects the gray scale values $\mathrm{P}_{(\mathrm{i}, \mathrm{j})}$ from intervals $0 \leq \mathrm{P}_{(\mathrm{i}, \mathrm{j})}<2^{\mathrm{n}-2}$ and from $15 \times 2^{\mathrm{n}-2}<\mathrm{P}_{(\mathrm{i}, \mathrm{j})}<256$ as shown in Fig. 13 and 14 . While almost of gray scale images are out of these intervals. Our proposed algorithm minimized the embedding error from $\left(2^{\mathrm{n}-1}\right)$ in the maximum pyramid of embedding error to the medial pyramid of embedding error $\left(2^{\mathrm{n}-2}\right)$ as shown in Fig. 16, then the summation number of embedding errors ' $\mathrm{i}$ ' are start from one to $\left(2^{n-2}\right)$ are growth one by one, the formula will be useful to calculate the summation of embedding errors ' $i$ ':

$$
\sum_{i=1}^{2^{n-2}} \mathrm{i}=1+2+\ldots+2^{\mathrm{n}-2}=\frac{2^{\mathrm{n}-2}\left(2^{\mathrm{n}-2}+1\right)}{2}=2^{\mathrm{n}-3}\left(2^{\mathrm{n}-2}+1\right)
$$

Hence from Eq. $4 c$ the summation of embedding errors ' $i$ ' can be calculated the average of embedding errors between the cover image and watermarked image derived by:

The average of embedding error $=\frac{2^{\mathrm{n}-3}\left(2^{\mathrm{n}-2}+1\right)}{2^{\mathrm{n}-2}}=\frac{2^{\mathrm{n}-2}+1}{2}$

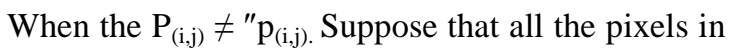
the cover image are used for the embedding of watermark bit by proposed method, theoretically, the average of worst mean square error between the cover image and watermarked image can be derived by Eq. 2c:

$$
\operatorname{Averg} \cdot \operatorname{WMSE}^{*}=\frac{1}{\mathrm{M} \times \mathrm{N}} \sum_{\mathrm{i}=1}^{\mathrm{M}} \sum_{\mathrm{j}=1}^{\mathrm{N}}\left[\frac{2^{\mathrm{n}-2}+1}{2}\right]^{2}=\left[\frac{2^{\mathrm{n}-2}+1}{2}\right]^{2}
$$

Notice that the WMSE $=\left(2^{\mathrm{n}-1}\right)^{2}$ by the direct replacement by the simple $\mathrm{LSB}_{\mathrm{n}}$ and $\mathrm{MSB}_{\mathrm{n}}$ substitution method are constant of embedded error $=2^{\mathrm{n}-1}$. But with proposed method (APAP-MPOEE-FOBCB ${ }_{\mathrm{MSB} 6}$ ) the max-worst mean square error Max.WMSE ${ }^{*}=\left(2^{\mathrm{n}-2}\right)^{2}$, Min. WMSE $^{*}=1$ and the average worst mean square error are obtained in Eq. 4e. Moreover the embedding errors are minimized to the half $(1 / 2)$ from the maxembedding errors $\Omega$ in the watermarked image as shown in Fig. 16. Let's WMSE and Max.WMSE* be the worst mean square error between watermarked image and cover image are obtained the WMSE = $\left(2^{\mathrm{n}-1}\right)^{2}$ by the direct replacement of simple $\mathrm{LSB}_{\mathrm{n}}$ or $\mathrm{MSB}_{\mathrm{n}}$ substitution and the Max.WMSE${ }^{*}$ by proposed method (APAP-MPOEE-FOBCB ${ }_{\text {MSB }}$ ). Theoretically, by combining WMSE and Max.WMSE*, we have:

$$
\frac{\text { Max.WMSE }^{*}}{\text { WMSE }}=\frac{\left(2^{\mathrm{n}-2}\right)^{2}}{\left(2^{\mathrm{n}-1}\right)^{2}}
$$

Max.WMSE ${ }^{*}=\frac{\text { Max.WMSE }}{\text { WMSE }}$ WMSE

$\operatorname{Max} \mathrm{WMSE}^{*}=\frac{\left(2^{\mathrm{n}-2}\right)^{2}}{\left(2^{\mathrm{n}-1}\right)^{2}}$ WMSE

From Eq. $4 \mathrm{~h}$ and when $\mathrm{n}=6$ reveals that the Max.WMSE ${ }^{*}=\frac{1}{4}$ WMSE,$\quad$ averg. WMSE $^{*}=0.0705$ WMSE and the Min.WMSE ${ }^{*}=\frac{1}{1024}$ WMSE, this result of our 
analyzed shows that the average of embedding errors in Eq. $4 \mathrm{~d}=8.5$ and WMSE* obtained by proposed scheme are proved efficient and better than obtained by the previous methods and modified algorithms.

\section{RESULTS AND DISCUSSUON}

The experimental results have been computed and applying to measure the performance result by comparative study between the previous methods, modified algorithms and proposed method (APAPMPOEE $_{M S B 6}$ ) in two parts with discussion (i): Theoretically analysis and (ii): Applied on the different benchmark of six-test-images and two quantum of watermark bit embedded, to study the performance of enhancement grey scale image quality means (fidelity), imperceptibility, capacity and robustness under of the mechanism different image attacks.

The experimental result computed theoretically: Experimental results have been computed theoretically. Suppose that all the pixels in the cover image are used for the embedding of watermark bit by the list of substitutions of previous methods, modified algorithms and proposed method (APAP-MPOEE ${ }_{\mathrm{MSB}}$ ), theoretically, have been measure in the Max, Min and the average number of embedding errors $\Omega$, worst mean square error WMSE, WMSE* and worst Peak Signal to Noise Ratio (PSNR ${ }_{\text {worst }}$ ) between the cover image $F_{(i, j)}$ and watermarked image $f_{(i, j)}$. Have been seen that the Table 1-4 tabulates the comparisons results. Furthermore, in the worst number of embedding errors
$\Omega$, there are only 256 possible pixel values for eight-bit gray scale images. Theoretically, in the Table 1 tabulates the number of embedding errors $\Omega$ for some of $n=1$ to eight-bit of gray scale images in $\left(\mathrm{LSB}_{n}\right.$ and $\mathrm{MSB}_{\mathrm{n}}$ ). It could be seen that the image quality of the watermarked image is degraded drastically when $n$ growth up one by one. In this letter, the number of embedding errors $\Omega$ in proposed method set of $\mathrm{MSB}_{6}=$ $16_{\text {Max }}, 8.5_{\text {avrg }}$ and $1_{\text {Min }}$ are lowest than with compared of the list substitutions of previous methods and modified algorithms. Moreover in the proposed method, the embedding errors are minimized to the half $1 / 2$ from the maximum embedding errors $\Omega=2^{\mathrm{n}-1}=32$ in the watermarked image as shown in Table 1 theoretically. However the worst mean square error WMSE and WMSE*, theoretically calculated in the Table 2 tabulates the WMSE for all eight-bit of gray scale images in $\left(\mathrm{LSB}_{\mathrm{n}}\right.$ and $\left.\mathrm{MSB}_{\mathrm{n}}\right)$. It could be seen that the image quality of the watermarked image is degraded drastically when growth depth in the $\mathrm{MSB}_{\mathrm{n}}$ one by one. In this letter the $\mathrm{WMSE}^{*}$ in proposed method set of $\mathrm{MSB}_{6}=256_{\mathrm{Max}}, 72.25_{\text {avrg }}$ and $1_{\text {Min }}$ are lowest with compared of the list substitutions of previous methods. On the other hand, let the worst mean square error WMSE obtained by simple $\mathrm{LSB}_{\mathrm{n}}$ or $\mathrm{MSB}_{\mathrm{n}}$ substitution method equal $=\left(2^{\mathrm{n}-1) 2}\right.$ and the simple $\mathrm{k}$-LSB substitution method using ' $\mathrm{k}$ ' capacity of embedded watermark bits $=\left(2^{\mathrm{k}-1}\right)^{2}$ as shown in Table 2. Furthermore the WMSE* obtained by the list substitutions of previous methods and proposed method, it is be the worst mean square error between the watermarked image and cover image as shown in Table 2.

Table 1: The number of embedding errors are compared between the proposed method, previous methods and modified algorithms

\begin{tabular}{|c|c|c|c|c|c|c|c|c|c|c|}
\hline \multirow{2}{*}{$\begin{array}{l}\text { Comparison between methods } \\
\text { of embedded watermark bits: }\end{array}$} & \multirow[b]{2}{*}{ Level } & \multirow{2}{*}{$\begin{array}{l}\text { Formula of the number } \\
\text { of embedding errors } \\
1<\mathrm{n} \leq 8, \mathrm{P}_{(\mathrm{i}, \mathrm{j})} \neq \mathrm{P}_{(\mathrm{i}, \mathrm{j})}^{\prime}\end{array}$} & \multicolumn{8}{|c|}{$\begin{array}{l}\text { There are only } 256 \text { possible pixel values for eight-bit gray scale image. } \\
\text { Thus the theatrically analysis of the number of embedding errors are: }\end{array}$} \\
\hline & & & $\mathrm{LSB}_{1}$ & $\mathrm{LSB}_{2}$ & $\mathrm{LSB}_{3}$ & $\mathrm{LSB}_{4}$ & $\mathrm{MSB}_{5}$ & $\mathrm{MSB}_{6}$ & $\mathrm{MSB}_{7}$ & $\mathrm{MSB}_{8}$ \\
\hline Simple LSB or MSB substation method & Constant & & 1 & 2.0 & 4.0 & 8.0 & 16.0 & 32.0 & 64.0 & 128.0 \\
\hline Methods of Wang-Lin-Lin & $\operatorname{Max}$ & $2^{\mathrm{n}-1}$ & 1 & 2.0 & 4.0 & 8.0 & 16.0 & 32.0 & 64.0 & 128.0 \\
\hline scheme and PAP-algorithm-1 & Average & $\frac{2\left(2^{n-1}-2^{n-3}+1\right)-1}{2}$ & 1 & 2.0 & 3.5 & 6.5 & 12.5 & 24.5 & 48.5 & 96.5 \\
\hline set of $\mathrm{MSB}_{6}$ & Min & $2^{\mathrm{n}-2}+1$ & 1 & 2.0 & 3.0 & 5.0 & 9.0 & 17.0 & 33.0 & 65.0 \\
\hline $\begin{array}{l}\text { Simple k-LSB substitution method using } \\
\text { 'k' capacity of embedded watermark bits }\end{array}$ & Constant & $2^{\mathrm{k}}-1$ & 1 & 3.0 & 7.0 & 15.0 & 31.0 & 63.0 & 127.0 & 255.0 \\
\hline Method of Chi-Kwong-Cheng & $\operatorname{Max}$ & $2^{k-1}$ & 1 & 2.0 & 4.0 & 8.0 & 16.0 & 32.0 & 64.0 & 128.0 \\
\hline scheme, using 'k' capacity of & Average & $\frac{2^{k-1}+1}{2}$ & 1 & 1.5 & 2.5 & 4.5 & 8.5 & 16.5 & 32.5 & 64.5 \\
\hline embedded watermark bits in k-LSB & Min & 1 & 1 & 1.0 & 1.0 & 1.0 & 1.0 & 1.0 & 1.0 & 1.0 \\
\hline PAP-algorithm-2 set of $\mathrm{MSB}_{6}$ & Constant & $2^{\mathrm{n}-1}$ & 1 & 2.0 & 4.0 & 8.0 & 16.0 & 32.0 & 64.0 & 128.0 \\
\hline Method of Aiad and Abdul scheme & $\operatorname{Max}$ & $2^{\mathrm{n}-1}$ & 1 & 2.0 & 4.0 & 8.0 & 16.0 & 32.0 & 64.0 & 128.0 \\
\hline and PAP-algorithm-3 set of $\mathrm{MSB}_{6}$ & Average & $\frac{2^{n-1}+1}{2}$ & 1 & 1.5 & 2.5 & 4.5 & 8.5 & 16.5 & 32.5 & 64.5 \\
\hline & Min & 1 & 1 & 1.0 & 1.0 & 1.0 & 1.0 & 1.0 & 1.0 & 1.0 \\
\hline Proposed method & Max & $2^{\mathrm{n}-2}$ & 1 & 1.0 & 2.0 & 4.0 & 8.0 & 16.0 & 32.0 & 64.0 \\
\hline & Average & $\frac{2^{\mathrm{n}-2}+1}{2}$ & 1 & 1.0 & 1.5 & 2.5 & 4.5 & 8.5 & 16.5 & 32.5 \\
\hline & Min & 1 & 1 & 1.0 & 1.0 & 1.0 & 1.0 & 1.0 & 1.0 & 1.0 \\
\hline
\end{tabular}


Am. J. Applied Sci., 7 (7): 987-1022, 2010

Table 2: Theoretically the WMSE and WMSE ${ }^{*}$ with comparisons

\begin{tabular}{|c|c|c|c|c|c|c|c|c|c|c|}
\hline \multirow{2}{*}{$\begin{array}{l}\text { Comparison between methods } \\
\text { of embedded watermark bits: }\end{array}$} & \multirow[b]{2}{*}{ Level } & \multirow{2}{*}{$\begin{array}{l}\text { Formula of the Worst } \\
\text { Mean Square Error of } \\
\text { embedding watermark } \\
\text { bits }\left(\mathrm{WMSE}^{*}\right) \text {, when } \\
1<\mathrm{n} \leq 8 \text { and } \mathrm{P}_{(\mathrm{i}, \mathrm{j})} \neq \mathrm{P}_{(\mathrm{i}, \mathrm{j})}^{\prime}\end{array}$} & \multicolumn{8}{|c|}{$\begin{array}{l}\text { Suppose that all the pixels in the cover image are used for embedded } \\
\text { watermark bits by the list of substation method. Thus the theatrically } \\
\text { analysis of the worst mean square(WMSE and WMSE*) are: }\end{array}$} \\
\hline & & & $\mathrm{LSB}_{1}$ & $\mathrm{LSB}_{2}$ & $\mathrm{LSB}_{3}$ & $\mathrm{LSB}_{4}$ & $\mathrm{MSB}_{5}$ & $\mathrm{MSB}_{6}$ & $\mathrm{MSB}_{7}$ & $\mathrm{MSB}_{8}$ \\
\hline Simple LSB or MSB substation method & Constant & $\left(2^{n-1}\right)^{2}$ & 1 & 4.00 & 16.00 & 64.00 & 256.00 & 1024.00 & 4096.00 & 16384.00 \\
\hline Methods of Wang-Lin-Lin & Max & $\left(2^{n-1}\right)^{2}$ & 1 & 4.00 & 16.00 & 64.00 & 256.00 & 1024.00 & 4096.00 & 16384.00 \\
\hline scheme and PAP-algorithm-1 & Average & {$\left[\frac{2\left(2^{\mathrm{n}-1}-2^{\mathrm{n}-3}+1\right)-1}{2}\right]$} & 1 & 4.00 & 12.25 & 42.25 & 156.25 & 600.25 & 2352.25 & 9312.25 \\
\hline set of $\mathrm{MSB}_{6}$ & Min & $\left(2^{\mathrm{n}-2}+1\right)^{2}$ & 1 & 4.00 & 9.00 & 25.00 & 81.00 & 289.00 & 1089.00 & 4225.00 \\
\hline $\begin{array}{l}\text { Simple k-LSB substitution method using } \\
\text { 'k' capacity of embedded watermark bits }\end{array}$ & Constant & $\left(2^{\mathrm{k}}-1\right)^{2}$ & 1 & 9.00 & 49.00 & 225.00 & 961.00 & 3969.00 & 16129.00 & 65025.00 \\
\hline Method of Chi-Kwong-Cheng & $\operatorname{Max}$ & $\left(2^{k-1}\right)^{2}$ & 1 & 4.00 & 16.00 & 64.00 & 256.00 & 1024.00 & 4096.00 & 16384.00 \\
\hline scheme, using ' $k$ ' capacity of & Average & {$\left[\frac{2^{k-1}+1}{2}\right]^{2}$} & 1 & 2.25 & 6.25 & 20.50 & 72.25 & 272.25 & 1056.25 & 4160.25 \\
\hline embedded watermark bits in k-LSB & Min & 1 & 1 & 1.00 & 1.00 & 1.00 & 1.00 & 1.00 & 1.00 & 1.00 \\
\hline PAP-algorithm-2 set of $\mathrm{MSB}_{6}$ & Constant & $\left(2^{\mathrm{n}-1}\right)^{2}$ & 1 & 4.00 & 16.00 & 64.00 & 256.00 & 1024.00 & 4096.00 & 16384.00 \\
\hline Method of Aiad and Abdul scheme & Max & $\left(2^{\mathrm{n}-1}\right)^{2}$ & 1 & 4.00 & 16.00 & 64.00 & 256.00 & 1024.00 & 4096.00 & 16384.00 \\
\hline and PAP-algorithm- 3 set of $\mathrm{MSB}_{6}$ & Average & {$\left[\frac{2^{\mathrm{n}-1}+1}{2}\right]^{2}$} & 1 & 2.25 & 6.25 & 20.25 & 72.25 & 272.25 & 1056.25 & 4160.25 \\
\hline \multirow{4}{*}{ Proposed method } & Min & & 1 & 1.00 & 1.00 & 1.00 & 1.00 & 1.00 & 1.00 & 1.00 \\
\hline & Max & $\left(2^{\mathrm{n}-2}\right)^{2}$ & 1 & 1.00 & 4.00 & 16.00 & 64.00 & 256.00 & 1024.00 & 4096.00 \\
\hline & Average & {$\left[\frac{2^{\mathrm{n}-2}+1}{2}\right]^{2}$} & 1 & 1.00 & 2.25 & 6.25 & 20.25 & 72.25 & 272.25 & 1056.25 \\
\hline & Min & 1 & 1 & 1.00 & 1.00 & 1.00 & 1.00 & 1.00 & 1.00 & 1.00 \\
\hline
\end{tabular}

Table 3: The combining WMSE within WMSE ${ }^{*}$ and comparisons

\begin{tabular}{|c|c|c|c|c|c|c|c|c|c|c|}
\hline \multirow{2}{*}{$\begin{array}{l}\text { Comparison between methods } \\
\text { of embedded watermark bits: }\end{array}$} & \multirow[b]{2}{*}{ Level } & \multirow{2}{*}{$\begin{array}{l}\text { Formula of the Worst } \\
\text { Mean Square Error } \\
\text { (WMSE*), when } \\
1<\mathrm{n} \leq 8 \text { and } \mathrm{P}_{(\mathrm{i}, \mathrm{j})} \neq \mathrm{P}_{(\mathrm{i}, \mathrm{j})}^{\prime}\end{array}$} & \multicolumn{8}{|c|}{$\begin{array}{l}\text { WMSE }=\left(2^{\mathrm{n}-1}\right)^{2} \text { or }\left(2^{\mathrm{k}}-1\right)^{2} \mathrm{WMSE}^{*}=\frac{\mathrm{WMSE}^{*}}{\mathrm{WMSE}^{*}} \mathrm{WMSE}^{\text {Wisin }} \\
\text { theoretically analysis of the combining WMSE within WMSE } \text { are: }\end{array}$} \\
\hline & & & $\mathrm{LSB}_{1}$ & $\mathrm{LSB}_{2}$ & $\mathrm{LSB}_{3}$ & $\mathrm{LSB}_{4}$ & $\mathrm{MSB}_{5}$ & $\mathrm{MSB}_{6}$ & $\mathrm{MSB}_{7}$ & $\mathrm{MSB}_{8}$ \\
\hline Methods of Wang-Lin-Lin & Max & $\left(2^{\mathrm{n}-1}\right)^{2}$ & WMSE & WMSE & WMSE & WMSE & WMSE & WMSE & WMSE & WMSE \\
\hline scheme and PAP-algorithm-1 & Average & {$\left[\frac{2\left(2^{\mathrm{n}-1}-2^{\mathrm{n}-3}+1\right)-1}{2}\right]$} & 1 & 1.0000 & 0.7656 & 0.6601 & 0.6103 & 0.5861 & 0.5742 & 0.5683 \\
\hline $\begin{array}{l}\text { set of } \mathrm{MSB}_{6} \\
\text { Method of Chi-Kwong-Cheng }\end{array}$ & $\begin{array}{l}\text { Min } \\
\text { Max }\end{array}$ & $\begin{array}{l}\left(2^{\mathrm{n}-2}+1\right)^{2} \\
\left(2^{\mathrm{k}-1}\right)\end{array}$ & $\begin{array}{l}1 \\
1\end{array}$ & $\begin{array}{l}1.0000 \\
0.4440\end{array}$ & $\begin{array}{l}0.5625 \\
0.3265\end{array}$ & $\begin{array}{l}0.3906 \\
0.2844\end{array}$ & $\begin{array}{l}0.3164 \\
0.2663\end{array}$ & $\begin{array}{l}0.2822 \\
0.2579\end{array}$ & $\begin{array}{l}0.2658 \\
0.2539\end{array}$ & $\begin{array}{l}0.2578 \\
0.2519\end{array}$ \\
\hline scheme, using ' $k$ ' capacity of & Average & {$\left[\frac{2^{k-1}+1}{2}\right]^{2}$} & 1 & 0.2500 & 0.1275 & 0.0900 & 0.0751 & 0.0685 & 0.0654 & 0.0639 \\
\hline embedded watermark bits in k-LSB & Min & 1 & 1 & & & & 0.00 & & & $1.53 \mathrm{E}-05$ \\
\hline PAP-algorithm-2 set of $\mathrm{MSB}_{6}$ & Constant & $\left(2^{n-1}\right)^{2}$ & WMSE & WMSE & WMSE & WMSE & WMSE & WMSE & WMSE & $\begin{array}{l}\text { WMSE } \\
\text { WMSE }\end{array}$ \\
\hline and PAP-algorithm- 3 set of $\mathrm{MSB}_{6}$ & Average & $\begin{array}{l}\left(2^{n-1}\right)^{2} \\
{\left[\frac{2^{n-1}+1}{2}\right]^{2}}\end{array}$ & WMSE & WMSE & WMSE & WMSE & WMSE & WMSE & WMSE & WMSE \\
\hline Proposed method & $\begin{array}{l}\text { Min } \\
\text { Max }\end{array}$ & $\begin{array}{l}1 \\
\left(2^{n-2}\right)^{2}\end{array}$ & $\begin{array}{l}1 \\
1\end{array}$ & $\begin{array}{l}0.2500 \\
0.2500\end{array}$ & $\begin{array}{l}0.0625 \\
0.2500\end{array}$ & $\begin{array}{l}0.0156 \\
0.2500\end{array}$ & $\begin{array}{l}0.0039 \\
0.2500\end{array}$ & $\begin{array}{l}0.0009 \\
0.2500\end{array}$ & $\begin{array}{l}0.0002 \\
0.2500\end{array}$ & $\begin{array}{l}6.10 \mathrm{E}-05 \\
0.2500\end{array}$ \\
\hline & Average & {$\left[\frac{2^{\mathrm{n}-2}+1}{2}\right]^{2}$} & 1 & 0.2500 & 0.1406 & 0.0976 & 0.0791 & 0.0705 & 0.0664 & 0.0644 \\
\hline & Min & 1 & 1 & 0.2500 & 0.0625 & 0.0156 & 0.0039 & 0.0009 & 0.0002 & $6.10 \mathrm{E}-05$ \\
\hline
\end{tabular}

Theoretically are computed in the Table 3, tabulates the combining between the WMSE and $\mathrm{WMSE}^{*}$ for all eight-bit of gray scale images in $\left(\mathrm{LSB}_{n}\right.$ and $\left.\mathrm{MSB}_{\mathrm{n}}\right)$, reveals that $\mathrm{WMSE}^{*}=\frac{\mathrm{WMSE}^{*}}{\mathrm{WMSE}} \mathrm{WMSE}$. It could be seen that in the $\mathrm{MSB}_{6}$ the combining are reveals that the
Max.WMSE* = 1/4WMSE, averg.WMSE* = 0.0705WMSE and Min.WMSE* ${ }^{*}=\frac{1}{1024}$ WMSE , obtained by the proposed method is better than that obtained by the list substitutions of previous methods and modified algorithms as shown in Table 3. 
Am. J. Applied Sci., 7 (7): 987-1022, 2010

Table 4: The worst cases of PSNR worst $_{\text {and comparison }}$

\begin{tabular}{|c|c|c|c|c|c|c|c|c|c|c|}
\hline \multirow{2}{*}{$\begin{array}{l}\text { Comparison between methods } \\
\text { of embedded watermark bits: }\end{array}$} & \multirow[b]{2}{*}{ Level } & \multirow{2}{*}{$\begin{array}{l}\text { Formula of the Worst } \\
\text { Mean Square Error of } \\
\text { embedding watermark } \\
\text { bits (WMSE*), when } \\
1<\mathrm{n} \leq 8 \text { and } \mathrm{P}_{(\mathrm{i}, \mathrm{j})} \neq \mathrm{P}_{(\mathrm{i}, \mathrm{j})}^{\prime}\end{array}$} & \multicolumn{8}{|c|}{$\begin{array}{l}\text { Suppose that all the pixels in the cover image are used for embedded } \\
\text { watermark bits by the list of substation method. Thus the theatrically analysis } \\
\text { in the worst PSNR (dB) by Eq. } 2 \mathrm{~g} \text { are: }\end{array}$} \\
\hline & & & $\mathrm{LSB}_{1}$ & $\mathrm{LSB}_{2}$ & $\mathrm{LSB}_{3}$ & $\mathrm{LSB}_{4}$ & $\mathrm{MSB}_{5}$ & $\mathrm{MSB}_{6}$ & $\mathrm{MSB}_{7}$ & $\mathrm{MSB}_{8}$ \\
\hline Simple LSB or MSB substation method & Constant & $\left(2^{\mathrm{n}-1}\right)^{2}$ & 48.130 & 42.110 & 36.089 & 30.069 & 24.048 & 18.027 & 12.007 & 5.9866 \\
\hline Methods of Wang-Lin-Lin & Max & $\left(2^{n-1}\right)^{2}$ & 48.130 & 42.110 & 36.089 & 30.069 & 24.048 & 18.027 & 12.007 & 5.9866 \\
\hline scheme and PAP-algorithm-1 & Average & {$\left[\frac{2\left(2^{\mathrm{n}-1}-2^{\mathrm{n}-3}+1\right)-1}{2}\right]$} & 48.130 & 42.110 & 37.249 & 31.872 & 26.192 & 20.347 & 14.4150 & 8.4402 \\
\hline $\begin{array}{l}\text { set of } \mathrm{MSB}_{6} \\
\text { Simple k-LSB substitution method using }\end{array}$ & Min & $\left(2^{\mathrm{n}-2}+1\right)^{2}$ & 48.130 & 42.110 & 38.558 & 34.151 & 29.045 & 23.521 & 17.7600 & $\begin{array}{r}11.8720 \\
0.0000\end{array}$ \\
\hline $\begin{array}{l}\text { Simple k-LSB substitution method using } \\
\text { 'k' capacity of embedded watermark bits }\end{array}$ & Constant & $\left(2^{k}-1\right.$ & & 38.588 & 31.228 & 24.608 & 18.303 & & 6.0540 & 0.0000 \\
\hline Method of Chi-Kwong-Cheng & Max & $\left(2^{k-1}\right)^{2}$ & 48.130 & 42.110 & 36.089 & 30.069 & 24.048 & 18.027 & 12.0072 & 5.9866 \\
\hline scheme, using 'k' capacity of & Average & {$\left[\frac{2^{k-1}+1}{2}\right]^{2}$} & 48.130 & 44.608 & 40.172 & 35.066 & 29.542 & 23.781 & 17.8930 & 11.9390 \\
\hline embedded watermark bits in k-LSB & Min & 1 & & & & 48.130 & & 48.130 & & 48.1300 \\
\hline PAP-a & Constant & $\left(2^{n-1}\right)^{2}$ & & & & & & & & 5.9866 \\
\hline Method of Aiad and Abdul scheme and & $\operatorname{Max}$ & $\left(2^{n-1}\right)^{2}$ & 48.130 & 42.110 & 36.089 & 30.069 & 24.048 & 18.027 & 12.0072 & 5.9866 \\
\hline PAP-algorithm- 3 set of $\mathrm{MSB}_{6}$ & Average & {$\left[\frac{2^{\mathrm{n}-1}+1}{2}\right]^{2}$} & 48.130 & 44.608 & 40.172 & 35.066 & 29.542 & 23.781 & 17.8930 & 11.9390 \\
\hline \multirow{4}{*}{ Proposed method } & Min & & 48.1 & 48.1 & 48. & 48.1 & & 48. & 48.1300 & 48.1300 \\
\hline & Max & $\left(2^{\mathrm{n}-2}\right)^{2}$ & 48.130 & 48.130 & 42.110 & 36.089 & 30.069 & 24.048 & 18.0270 & 12.0070 \\
\hline & Average & {$\left[\frac{2^{\mathrm{n}-2}+1}{2}\right]^{2}$} & 48.130 & 48.130 & 44.608 & 40.172 & 35.066 & 29.542 & 23.7811 & 17.8931 \\
\hline & Min & 1 & 48.130 & 48.130 & 48.130 & 48.130 & 48.130 & 48.130 & 48.1300 & 48.1300 \\
\hline
\end{tabular}

Finally, the Table 4 tabulates the worst case of $\mathrm{PSNR}_{\text {worst(dB) }}$ for each $\mathrm{LSB}_{\mathrm{n}}$ and $\mathrm{MSB}_{\mathrm{n}}$ of gray scale image. It could be seen that the image quality of the watermarked image is degraded drastically when $n$ growth one by one initial to the eight-bit. Hence that theoretically, the worst case of PSNR worst $_{\text {in }}$ proposed method set of $\mathrm{MSB}_{6}=\left(24.048_{(\mathrm{dB})}\right)_{\mathrm{Max}},\left(29.542_{(\mathrm{dB})}\right)_{\text {avg }}$ and $\left(48.130_{(\mathrm{dB})}\right)_{\mathrm{Min}}$ are higher than with compared of the list substitutions of previous methods and modified

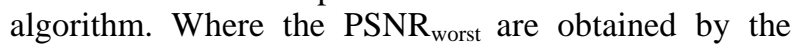
proposed method is better than that obtained by the list substitutions of previous methods and modified algorithm as shown in Table 4. Furthermore, the proposed method of an adaptively pixel adjustment process based on medial pyramid of embedding error set of the Most-Significant-Bit-n (APAP-MPOEE $\mathrm{MSB}$ ), the algorithm is requires a checking between the $\mathrm{MSB}_{6}$ in the pixel of cover image and EMB of the embedded watermark bit before embedding the watermark bits depending on the nearest of adaptively pixel in the medial pyramid of embedding error to inform the watermarked image " $\mathrm{P}_{(\mathrm{i}, \mathrm{j})}$. Thus theoretically are proved efficient and better than obtained by the previous methods and modified algorithms.

The experimental result applied on different benchmark: The experimental results have been applied on different benchmark six-test-images (Lena, Boat, Baboon, jet, Birds and Pills) to study the performance of enhancement grey scale image quality (fidelity), imperceptibility, capacity and robustness under of the mechanism different image attacks. In order to compare the performance results of the proposed novel method APAP-MPOEE set-of-the $\mathrm{MSB}_{6}$ by applying falling-off-boundary in corners board of cover image with random pixel manipulation in spatial domain (APAP-MPOEE-FOBCB ${ }_{\mathrm{MSB}}$ ), with the state-of-the-art-algorithms are required, Kevin et al. (2005) proposed an investigation into the use LSB substitution in digital watermarking by simple $\mathrm{MSB}_{6}$ substitution, Wang et al. (2000) proposed hiding data in images by optimal moderately significant bit replacement used $\mathrm{LSB}_{4}$, Chan and Cheng (2004) proposed hiding data in images by simple LSB substitution using k-right most LSBs substitution by applying OPAP, Aiad and Sada (2007) proposed hiding data using $\mathrm{LSB}_{3}$ by applying LPAP and our method of robust digital watermarking based falling-off-boundary in corners board gray scale images (Mehemed et al., 2009), by the way will be compare the modified algorithms of previous methods (PAP-algorithm-1, PAP-algorithm-2 and PAP-algorithm-3) by applying the falling-off-boundary in corners board of cover image set-of-the $\mathrm{MSB}_{6}$ with random pixel manipulation in spatial domain with proposed method. A set of standard six-test grey scale images (Lena, Boat, Baboon, jet, Birds and Pills) $512 \times 512$ gray scale level images has been used as a cover images as shown in Table 5 and two quantum of watermark bit embedded, by using a different size of binary watermark image $45 \times 45$ and $16 \times 16$ as shown in Fig. 17, are used to tested the performance of capacity, by insertion with 
redundantly distributed the watermark bits over many pixels of the cover image, by using a small binary watermark image $16 \times 16$, are added simultaneously in the falling-off-boundary in corners board pixel in the cover image as a capacity data bits to increase watermark intensity as a power of the embedded watermark, this procedure maybe increasing the robustness, as well as the increasing the watermark intensity will be cause the cost of the degradation of watermarked image. However the max-bits can be embedded 2048 bits in the cover image.

Imperceptibility: To measure the performance and compare between the state-of-the-art algorithms are required (Kevin et al., 2005; Chan and Cheng, 2004; Aiad and Sada, 2007; Mehemed et al., 2009; Wang et al., 2000 ) and modified algorithms of previous methods. We evaluated imperceptibility to sense the degree of distortion resulting from pixel value changes in watermarked image $\mathrm{f}_{(\mathrm{M}, \mathrm{N})}$ by the most popular difference distortion measures of pixel based metrics. These measures metrics are all based on the difference between the cover image $\mathrm{F}_{(\mathrm{M}, \mathrm{N})}$ and watermarked image:

- Average absolute difference: $\mathrm{AD}$ is used to measure the variation of the embedding errors occurred by the embedded watermark bits between the cover images and watermarked image as shown in Table 5. Whereas the $\mathrm{AD}$ of the proposed method is equal (0.032777) average, where the proposed method are lowest than with compared between previous methods and modified algorithms. Since that in the modified algorithm (PAP-algorithm-3), the $\mathrm{AD}$ are equal $(0.068829)_{\text {average }}$, where are lower with compared of the previous methods. Thus the AD in the other methods of previous methods and modified algorithms are higher

- Normalized average absolute difference: NAD is used to measure the variation of changes the range of pixel intensity values between the cover image and watermarking image as shown in Table 6. Whereas in the proposed method the NAD = $(0.000253)_{\text {average, }}$ are the lowest than with compared of the list substitutions of previous methods and modified algorithms. Moreover the NAD of modified PAP-algorithm-3 is $(0.000532)_{\text {average, }}$ where the PAP-algorithm-3 is better than with compared of the other modified algorithms

- Mean square error: The MSE is the cumulative squared error between the watermarked image $\mathrm{f}_{(\mathrm{M}, \mathrm{N})}$ and the cover image $\mathrm{F}_{(\mathrm{M}, \mathrm{N})}$. The Table 7 tabulates the MSE, whereas a lower value for MSE is in proposed method equal $(0.358758)_{\text {average, }}$ where are lesser error with compared between previous methods and modified algorithms. Moreover the MSE of modified PAP-algorithm-3 is equal (1.551337) average, where the PAPalgorithm-3 is better than with compared of the other modified algorithms. Moreover the lower value of MSE, means that the lower the error

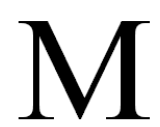

(a)-watermark $(16 \times 16)$

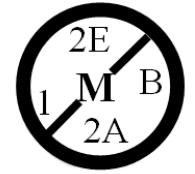

(a)-watermark $(45 \times 45)$
Fig. 17: The binary watermark image

Table 5: The performance results of $\mathrm{AD}$ with comparisons

\begin{tabular}{|c|c|c|c|c|c|c|}
\hline \multirow[b]{3}{*}{ Comparisons between state-of-the-art algorithm } & \multicolumn{6}{|c|}{ AD have been applied on different benchmark test images } \\
\hline & & & & & & Exy \\
\hline & Lena & Boat & Baboon & Jet & Birds & Pills \\
\hline Methods of Wang-Lin-Lin scheme & 1.168892 & 1.137920 & 1.142609 & 1.151752 & 1.136826 & 1.132618 \\
\hline Method of OPAP K-LSB $1, \mathrm{k}=1$ & 0.205574 & 0.205532 & 0.205383 & 0.205204 & 0.204319 & 0.204983 \\
\hline Method of OPAP K-LSB $1,2 \mathrm{k}=2$ & 0.290031 & 0.290611 & 0.289585 & 0.289799 & 0.290260 & 0.289192 \\
\hline Method of OPAP K-LSB $1,2,3 \mathrm{k}=3$ & 0.577080 & 0.581444 & 0.575672 & 0.579075 & 0.581032 & 0.572990 \\
\hline Method of OPAP K-LSB $1,2,3,4 \mathrm{k}=4$ & 1.169395 & 1.125023 & 1.144703 & 1.157658 & 1.149666 & 1.148590 \\
\hline Method of Aiad and Abdul scheme & 1.250572 & 1.247662 & 1.256798 & 1.263229 & 1.240578 & 1.255844 \\
\hline Method of an investigation into simple $\mathrm{MSB}_{6}$ substation & 16.025024 & 16.151730 & 15.819946 & 16.538330 & 16.013550 & 16.237183 \\
\hline Method, filling-of-boundary in corners bored set-of-MSB ${ }_{6}$ & 0.119873 & 0.121826 & 0.127075 & 0.122559 & 0.121948 & 0.125122 \\
\hline Applying method of optimal moderately by & 0.109798 & 0.111042 & 0.106998 & 0.094780 & 0.090996 & 0.098965 \\
\hline $\begin{array}{l}\text { FOBICB-set-of-MSB }{ }_{6} \text { and (PAP-algorithm-1) } \\
\text { Applying method of hiding data using FOBICB set }\end{array}$ & 0.061203 & 0.072075 & 0.068748 & 0.063946 & 0.076675 & 0.070324 \\
\hline of MSB $_{6}$ with modified LSB LS, $_{1,3,4}$, MSB $_{6}$ and (PAP-algorithm-3) & & & & & & \\
\hline $\begin{array}{l}\text { Applying method of OPAP algorithm by FOBICB-set- of- } \\
\text { MSB }_{6} \text { and (PAP-algorithm-2) }\end{array}$ & 0.119873 & 0.121826 & 0.127075 & 0.122559 & 0.121948 & 0.125122 \\
\hline $\begin{array}{l}\text { Proposed method of APAP-MPOEE applying filling-of- } \\
\text { boundary in corners bored set-of-MSB }\end{array}$ & 0.029156 & 0.036804 & 0.033241 & 0.030457 & 0.0340880 & 0.329170 \\
\hline
\end{tabular}


Am. J. Applied Sci., 7 (7): 987-1022, 2010

Table 6: The performance results of NAD with comparisons

\begin{tabular}{|c|c|c|c|c|c|c|}
\hline \multirow[b]{3}{*}{ Comparisons between state-of-the-art algorithm } & \multicolumn{6}{|c|}{ NAD have been applied on different benchmark test images } \\
\hline & & & & & 8.1. & Sys \\
\hline & Lena & Boat & Baboon & Jet & Birds & Pills \\
\hline Methods of Wang-Lin-Lin scheme & 0.009358 & 0.008795 & 0.008896 & 0.006500 & 0.010368 & 0.008925 \\
\hline method of OPAP K-LSB $1, \mathrm{k}=1$ & 0.001646 & 0.001589 & 0.001599 & 0.001158 & 0.001863 & 0.001609 \\
\hline method of OPAP K-LSB $1,2 \mathrm{k}=2$ & 0.002322 & 0.002246 & 0.002255 & 0.001636 & 0.002647 & 0.002270 \\
\hline method of OPAP K-LSB $1,2,3 \mathrm{k}=3$ & 0.004620 & 0.004494 & 0.004482 & 0.003268 & 0.005299 & 0.004499 \\
\hline method of OPAP K-LSB $1,2,3,4 \mathrm{k}=4$ & 0.009362 & 0.008696 & 0.008912 & 0.006534 & 0.010486 & 0.009018 \\
\hline Method of Aiad and Abdul scheme & 0.010012 & 0.009643 & 0.009785 & 0.007130 & 0.011315 & 0.009860 \\
\hline Method of an investigation into simple $\mathrm{MSB}_{6}$ substation & 0.128299 & 0.124840 & 0.123165 & 0.093341 & 0.146052 & 0.127481 \\
\hline Method, filling-of-boundary in corners bored set-of-MSB ${ }_{6}$ & 0.000960 & 0.000942 & 0.000989 & 0.000692 & 0.001112 & 0.000982 \\
\hline Applying method of optimal moderately by & 0.000879 & 0.000858 & 0.000833 & 0.000535 & 0.000830 & 0.000777 \\
\hline FOBICB-set-of-MSB 6 and (PAP-algorithm-1) & & & & & & \\
\hline $\begin{array}{l}\text { Applying method of hiding data using FOBICB set } \\
\text { of } \text { MSB }_{6} \text { with modified LSB } \\
1,2,3,4, \text { MSB }_{6} \text { and (PAP-algorithm-3) }\end{array}$ & 0.000490 & 0.000557 & 0.000535 & 0.000361 & 0.000699 & 0.000552 \\
\hline $\begin{array}{l}\text { Applying method of OPAP algorithm by FOBICB-set- of- } \\
\text { MSB }_{6} \text { and (PAP-algorithm-2) }\end{array}$ & 0.000960 & 0.000942 & 0.000989 & 0.000693 & 0.001112 & 0.000982 \\
\hline $\begin{array}{l}\text { Proposed method of APAP-MPOEE applying filling-of- } \\
\text { boundary in corners bored set-of-MSB }\end{array}$ & 0.000233 & 0.000284 & 0.000259 & 0.000172 & 0.000311 & 0.000258 \\
\hline
\end{tabular}

Table 7: The performance results of MSE with comparisons

\begin{tabular}{|c|c|c|c|c|c|c|}
\hline \multirow[b]{3}{*}{ Comparisons between state-of-the-art algorithm } & \multicolumn{6}{|c|}{ MSE have been applied on different benchmark test images } \\
\hline & & s & & & Sis & Ex: \\
\hline & Lena & Boat & Baboon & Jet & Birds & Pills \\
\hline Methods of Wang-Lin-Lin scheme & 8.3271260 & 8.021168 & 8.089958 & 8.163006 & 8.077568 & 8.037651 \\
\hline method of OPAP K-LSB $1, \mathrm{k}=1$ & 0.4587710 & 0.458591 & 0.457703 & 0.457867 & 0.454464 & 0.456493 \\
\hline method of OPAP K-LSB $1,2 \mathrm{k}=2$ & 1.3857420 & 1.390625 & 1.381290 & 1.387402 & 1.385101 & 1.377754 \\
\hline method of OPAP K-LSB $1,2,3 \mathrm{k}=3$ & 5.7503970 & 5.797272 & 5.735722 & 5.796764 & 5.802826 & 5.676796 \\
\hline method of OPAP K-LSB $1,2,3,4 \mathrm{k}=4$ & 23.8972470 & 22.528656 & 23.321171 & 23.569286 & 23.340851 & 23.173866 \\
\hline Method of Aiad and Abdul scheme & 3.7582860 & 3.740513 & 3.780121 & 3.822701 & 3.726509 & 3.771149 \\
\hline Method of an investigation into simple $\mathrm{MSB}_{6}$ substation & 512.8000781 & 516.855469 & 506.238281 & 529.226563 & 512.433594 & 519.589844 \\
\hline Method, filling-of-boundary in corners bored set-of-MSB ${ }_{6}$ & 3.8359380 & 3.898438 & 4.066406 & 3.921875 & 3.902344 & 4.003906 \\
\hline $\begin{array}{l}\text { Applying method of optimal moderately by } \\
\text { FOBICB-set-of-MSB }{ }_{6} \text { and (PAP-algorithm-1) }\end{array}$ & 3.2481800 & 3.270924 & 2.987247 & 2.434158 & 2.262512 & 2.617588 \\
\hline $\begin{array}{l}\text { Applying method of hiding data using FOBICB set } \\
\text { of } \text { MSB }_{6} \text { with modified LSB } \\
\text { L }_{1,2,3,4}, \text { MSB }_{6} \text { and (PAP-algorithm-3) }\end{array}$ & 1.3617400 & 1.584038 & 1.538620 & 1.429089 & 1.781166 & 1.613369 \\
\hline $\begin{array}{l}\text { Applying method of OPAP algorithm by FOBICB-set- of- } \\
\text { MSB }_{6} \text { and (PAP-algorithm-2) }\end{array}$ & 3.8359380 & 3.898438 & 4.066406 & 3.921875 & 3.902344 & 4.003906 \\
\hline $\begin{array}{l}\text { Proposed method of APAP-MPOEE applying filling-of- } \\
\text { boundary in corners bored set-of-MSB }\end{array}$ & 0.3041800 & 0.420105 & 0.366882 & 0.323944 & 0.375168 & 0.362270 \\
\hline
\end{tabular}

- Normalized mean square error: We know that the distance measure is normalized to a range between 0 and 1. It is independent of the range of gray scale values in the images. The Table 8 tabulates the NMSE, whereas the proposed method are get a great-ideal of NMSE equal (0.00001) average, with are compared of the list substitutions of the previous methods and modified algorithms. On the other hand the same case in the modified algorithms (PAP-algorith-3) equal (0.000083) average, where the proposed method is better than compared with each other methods. Here the NMSE of proposed method suggests the images are very similar in spatial layout and gray scale values

Signal to noise ratio: We know that the higher ratio means that the less obtrusive of the embedding errors in the watermarked image. Thus the Table 9 tabulates the SNR; the proposed method is getting a higher of SNR equal (47.34791367 dB) average, with compared of the list substitutions of the previous methods and modified algorithms. Moreover the SNR is a technical term used to characterize the quality of the watermarked image. On the other hand have been see that the modified algorithm (PAP-algorith-3) are get SNR

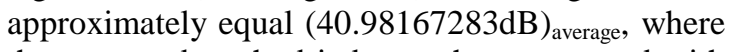
the proposed method is better than compared with each other methods. Here the SNR of proposed method suggests the Image Fidelity (IF) is very similar in spatial layout and gray scale values with the lowest distortion in gray scale image. Thus this is the prove imperceptibility and image fidelity of our proposed method, where are great higher SNR 
Table 8: The performance results of NMSE with comparisons

\begin{tabular}{|c|c|c|c|c|c|c|}
\hline \multirow[b]{3}{*}{ Comparisons between state-of-the-art algorithm } & \multicolumn{6}{|c|}{ NMSE have been applied on different benchmark test images } \\
\hline & & & & & 8 & Exy \\
\hline & Lena & Boat & Baboon & Jet & Birds & Pills \\
\hline Methods of Wang-Lin-Lin scheme & 0.000475 & 0.000428 & 0.000458 & 0.000245 & 0.000574 & 0.000413 \\
\hline method of OPAP K-LSB $1, \mathrm{k}=1$ & 0.000026 & 0.000024 & 0.000026 & 0.000014 & 0.000032 & 0.000023 \\
\hline method of OPAP K-LSB $1,2 \mathrm{k}=2$ & 0.000079 & 0.000074 & 0.000078 & 0.000042 & 0.000098 & 0.000071 \\
\hline method of OPAP K-LSB $1,2,3 \mathrm{k}=3$ & 0.000328 & 0.000309 & 0.000325 & 0.000174 & 0.000413 & 0.000292 \\
\hline method of OPAP K-LSB $1,2,3,4 \mathrm{k}=4$ & 0.001364 & 0.001202 & 0.001320 & 0.000707 & 0.001660 & 0.001191 \\
\hline Method of Aiad and Abdul scheme & 0.000214 & 0.000200 & 0.000214 & 0.000115 & 0.000265 & 0.000194 \\
\hline Method of an investigation into simple $\mathrm{MSB}_{6}$ substation & 0.029260 & 0.027574 & 0.028653 & 0.015884 & 0.036436 & 0.026711 \\
\hline Method, filling-of-boundary in corners bored set-of-MSB ${ }_{6}$ & 0.000219 & 0.000208 & 0.000230 & 0.000118 & 0.000277 & 0.000206 \\
\hline Applying method of optimal moderately by & 0.000185 & 0.000175 & 0.000169 & 0.000073 & 0.000161 & 0.000135 \\
\hline FOBICB-set-of-MSB 6 and (PAP-algorithm-1) & & & & & & \\
\hline $\begin{array}{l}\text { Applying method of hiding data using FOBICB set } \\
\text { of } \text { MSB }_{6} \text { with modified LSB } \text { L }_{1,2,3,4} \text { MSB }_{6} \text { and (PAP-algorithm-3) }\end{array}$ & 0.000078 & 0.000085 & 0.000087 & 0.000043 & 0.000127 & 0.000083 \\
\hline $\begin{array}{l}\text { Applying method of OPAP algorithm by FOBICB-set- of- } \\
\text { MSB }_{6} \text { and (PAP-algorithm-2) }\end{array}$ & 0.000219 & 0.000208 & 0.000230 & 0.000118 & 0.000277 & 0.000206 \\
\hline $\begin{array}{l}\text { Proposed method of APAP-MPOEE applying filling-of- } \\
\text { boundary in corners bored set-of-MSB }\end{array}$ & 0.000017 & 0.000022 & 0.000021 & 0.000010 & 0.000027 & 0.000019 \\
\hline
\end{tabular}

Table 9: The performance results of SNR with comparisons

\begin{tabular}{|c|c|c|c|c|c|c|}
\hline \multirow[b]{3}{*}{ Comparisons between state-of-the-art algorithm } & \multicolumn{6}{|c|}{ SNR have been applied on different benchmark test images } \\
\hline & 18 & & & & B., & oxy \\
\hline & Lena & Boat & Baboon & Jet & Birds & Pills \\
\hline Methods of Wang-Lin-Lin scheme & 33.231720 & 33.686246 & 33.392360 & 36.108440 & 32.408291 & 33.838476 \\
\hline Method of OPAP K-LSB,$k=1$ & 45.820713 & 46.114363 & 45.865988 & 48.619551 & 44.906103 & 46.295423 \\
\hline Method of OPAP K-LSB $1,2 \mathrm{k}=2$ & 41.019846 & 41.296522 & 41.068973 & 43.804919 & 40.066282 & 41.498050 \\
\hline Method of OPAP K-LSB ${ }_{1,2,3} \mathrm{k}=3$ & 34.839693 & 35.096386 & 34.885942 & 37.595085 & 33.844702 & 35.348735 \\
\hline Method of OPAP K-LSB $1,2,3,4 \mathrm{k}=4$ & 28.653192 & 29.201270 & 28.794319 & 31.503477 & 27.799930 & 29.239783 \\
\hline Method of Aiad and Abdul scheme & 36.686773 & 36.999311 & 36.696766 & 39.403238 & 35.768075 & 37.125031 \\
\hline Method of an investigation into simple $\mathrm{MSB}_{6}$ substation & 15.337184 & 15.594931 & 15.428273 & 17.990525 & 14.384721 & 15.733161 \\
\hline Method, filling-of-boundary in corners bored set-of-MSB 6 & 36.597955 & 36.819717 & 36.379715 & 39.292004 & 35.567842 & 36.864929 \\
\hline Applying method of optimal moderately by & 37.320269 & 37.581918 & 37.719111 & 41.363453 & 37.935188 & 38.710755 \\
\hline FOBICB-set-of-MSB 6 and (PAP-algorithm-1) & & & & & & \\
\hline $\begin{array}{l}\text { Applying method of hiding data using FOBICB set } \\
\text { of } \text { MSB }_{6} \text { with modified } \text { LSB }_{1,2,3,4}, \text { MSB }_{6} \text { and (PAP-algorithm-3) }\end{array}$ & 41.095728 & 40.730967 & 40.600509 & 43.676349 & 38.974053 & 40.812431 \\
\hline $\begin{array}{l}\text { Applying method of OPAP algorithm by FOBICB-set- of-MSB } 6 \\
\text { and (PAP-algorithm-2) }\end{array}$ & 36.597955 & 36.819717 & 36.379715 & 39.292004 & 35.567842 & 36.864929 \\
\hline $\begin{array}{l}\text { Proposed method of APAP-MPOEE applying filling-of-boundary } \\
\text { in corners bored set-of-MSB } 6\end{array}$ & 47.605362 & 46.495044 & 46.826555 & 50.122240 & 45.738841 & 47.299440 \\
\hline
\end{tabular}

Table 10: The performance results of PSNR with comparisons

\begin{tabular}{|c|c|c|c|c|c|c|}
\hline \multirow[b]{3}{*}{ Comparisons between state-of-the-art algorithm } & \multicolumn{6}{|c|}{ PSNR have been applied on different benchmark test images } \\
\hline & & & & & g.1. & Exy \\
\hline & Lena & Boat & Baboon & Jet & Birds & Pills \\
\hline Methods of Wang-Lin-Lin scheme & 38.925852 & 39.088428 & 39.051341 & 39.012303 & 39.057997 & 39.079512 \\
\hline method of OPAP K-LSB $1, \mathrm{k}=1$ & 51.402418 & 51.516544 & 51.524969 & 51.523413 & 51.555809 & 51.536459 \\
\hline method of OPAP K-LSB $1,2 \mathrm{k}=2$ & 46.703865 & 46.698703 & 46.727954 & 46.708782 & 46.715988 & 46.739086 \\
\hline method of OPAP K-LSB ${ }_{1,2,3} \mathrm{k}=3$ & 40.690663 & 40.498567 & 40.544923 & 40.498947 & 40.494408 & 40.589771 \\
\hline method of OPAP K-LSB $1,2,3,4 \mathrm{k}=4$ & 34.527257 & 34.603451 & 34.453300 & 34.407339 & 34.449637 & 34.480819 \\
\hline Method of Aiad and Abdul scheme & 42.290906 & 42.401492 & 42.355747 & 42.307101 & 42.417782 & 42.366067 \\
\hline Method of an investigation into simple $\mathrm{MSB}_{6}$ substation & 21.031317 & 20.997112 & 21.087254 & 20.894387 & 21.034428 & 20.974197 \\
\hline Method, filling-of-boundary in corners bored set-of-MSB 6 & 42.292088 & 42.221898 & 42.038696 & 42.195866 & 42.217548 & 42.105965 \\
\hline Applying method of optimal moderately by & 43.014402 & 42.984100 & 43.378092 & 44.267315 & 44.584894 & 43.951791 \\
\hline FOBICB-set-of-MSB 6 and (PAP-algorithm-1) & & & & & & \\
\hline $\begin{array}{l}\text { Applying method of hiding data using FOBICB set } \\
\text { of } \text { MSB }_{6} \text { with modified } \text { LSB }_{1,2,3,4}, \text { MSB }_{6} \text { and (PAP-algorithm-3) }\end{array}$ & 46.789861 & 46.133148 & 46.259490 & 46.580212 & 45.623759 & 46.053467 \\
\hline $\begin{array}{l}\text { Applying method of OPAP algorithm by FOBICB-set- of- } \\
\text { MSB }_{6} \text { and (PAP-algorithm-2) }\end{array}$ & 42.292088 & 42.221898 & 42.038696 & 42.195866 & 42.217548 & 42.105965 \\
\hline $\begin{array}{l}\text { Proposed method of APAP-MPOEE applying filling-of- } \\
\text { boundary in corners bored set-of-MSB } 6\end{array}$ & 53.299495 & 51.897225 & 52.485536 & 53.026103 & 52.388548 & 52.540476 \\
\hline
\end{tabular}


- Peak signal to noise ratio: We know that the typical PSNR values range between 20 and $40 \mathrm{~dB}$, where higher is better for quality image. Here in the Table 10 tabulates the PSNR, in the modified algorithm (PAP-algorithm-3), the PSNR are equal $(46.2399895 \mathrm{~dB})_{\text {average}}$, where are higher than with compared of the previous methods and modified algorithm. But in the proposed method is getting a higher of PSNR equal $(52.6062305 \mathrm{~dB})_{\text {average}}$, with compared of the list substitutions of the previous methods and modified algorithms. Thus where the quality degradations could hardly be perceived by human eye, then the PSNR of proposed method suggests the high quality image and Image Fidelity (IF), where are very similar closeness in spatial layout of gray scale values between cove image and watermarked image. So that this is the prove imperceptibility and image fidelity for our proposed method, where are greatens higher PSNR

Image fidelity: Have been see that the Table 11 tabulates the Image Fidelity (IF), in the modified algorithm (PAP-algorithm-3), the Image fidelity are equal $(0.999916167)_{\text {average, }}$ where are lower than with compared of the previous methods and modified algorithm. But on the other hand the image fidelity of the proposed method are getting ideal value equal $(0.999980667)_{\text {average, }}$ with compared of the list substitutions of the previous methods and modified algorithms. Thus where the quality degradations could hardly be perceived by human eye, then the image fidelity of proposed method suggests a high quality of watermarked image and higher imperceptibility.
Where are very similar in spatial layout of gray scale values between cove image and watermarked image. Furthermore the image fidelity measure is also commonly referred to as an image metric, the traditional image fidelity standard are the Mean Squared-Error (MSE), Signal-to-Noise Ratio (SNR) and the Peak Signal-to-Noise Ratio (PSNR) for cover image with watermarked image. From the Table 6-10 in proposed technique measuring, First: The measuring value of NAD equal $(0.000253)_{\text {average, }}$ are signifies lesser error in the watermarked image. Second: The MSE has been obtained a lowest average of the squared difference between the intensity of the cover image and watermarked image at each pixel location equal $(0.358758)_{\text {average, }}$ with ideal value in the NMSE equal $(0.00001)_{\text {average, }}$ Third: The SNR getting a higher ratio equal (47.34791367 dB) average, means that the lesser obtrusive of the embedding errors (called noise) in the watermarked image. Whereas the SNR is a technical term used to characterize the quality of the watermarked image detection. Four: The PSNR is getting a higher of PSNR equal $(52.6062305 \mathrm{~dB})_{\text {average, }}$, here a logically a higher value of PSNR is high quality watermarked image. Thus form our observation the values of (Image fidelity, NAD, MSE, NMSE, SNR and PSNR) in proposed method are demonstrates better performance than of the list substitutions of the previous methods and modified algorithms, these are proves of the imperceptibility, higher quality watermarked image means image fidelity of our proposed novel method as shown the watermarked image in the Fig. 18a-g shown the extracted watermark image using as logo/hidden.

Table 11: The performance results of image fidelity with comparisons

\begin{tabular}{|c|c|c|c|c|c|c|}
\hline \multirow[b]{2}{*}{ Comparisons between state-of-the-art algorithm } & \multicolumn{6}{|c|}{ Image fidelity have been applied on different benchmark test images } \\
\hline & Lena & Boat & Baboon & Jet & Birds & Pills \\
\hline Methods of Wang-Lin-Lin scheme & 0.999525 & 0.999572 & 0.999542 & 0.999755 & 0.999426 & 0.999587 \\
\hline Method of OPAP K-LSB $1, k=1$ & 0.999974 & 0.999976 & 0.999974 & 0.999986 & 0.999968 & 0.999977 \\
\hline Method of OPAP K-LSB ${ }_{1,2} \mathrm{k}=2$ & 0.999921 & 0.999926 & 0.999922 & 0.999958 & 0.999902 & 0.999929 \\
\hline Method of OPAP K-LSB $1,2,3 \mathrm{k}=3$ & 0.999672 & 0.999691 & 0.999675 & 0.999826 & 0.999587 & 0.999708 \\
\hline Method of OPAP K-LSB $1,2,3,4 \mathrm{k}=4$ & 0.998636 & 0.998798 & 0.998680 & 0.999293 & 0.998340 & 0.998809 \\
\hline Method of Aiad and Abdul scheme & 0.999786 & 0.999800 & 0.999786 & 0.999885 & 0.999735 & 0.999806 \\
\hline Method of an investigation into simple $\mathrm{MSB}_{6}$ substation & 0.970740 & 0.972426 & 0.971347 & 0.984116 & 0.963564 & 0.973289 \\
\hline Method, filling-of-boundary in corners bored set-of-MSB 6 & 0.999781 & 0.999792 & 0.999770 & 0.999882 & 0.999723 & 0.999794 \\
\hline Applying method of optimal moderately by & 0.999815 & 0.999825 & 0.999831 & 0.999927 & 0.999839 & 0.999865 \\
\hline FOBICB-set-of-MSB 6 and (PAP-algorithm-1) & & & & & & \\
\hline $\begin{array}{l}\text { Applying method of hiding data using FOBICB set } \\
\text { of } \mathrm{MSB}_{6} \text { with modified } \mathrm{LSB}_{1,2,3,4}, \mathrm{MSB}_{6} \text { and (PAP-algorithm-3) }\end{array}$ & 0.999922 & 0.999915 & 0.999913 & 0.999957 & 0.999873 & 0.999917 \\
\hline $\begin{array}{l}\text { Applying method of OPAP algorithm by FOBICB-set- of-MSB } 6 \\
\text { And (PAP-algorithm-2) }\end{array}$ & 0.999781 & 0.999792 & 0.999770 & 0.999882 & 0.999723 & 0.999794 \\
\hline $\begin{array}{l}\text { Proposed method of APAP-MPOEE applying filling-of-boundary } \\
\text { in corners bored set-of-MSB } 6\end{array}$ & 0.999983 & 0.999978 & 0.999979 & 0.999990 & 0.999973 & 0.999981 \\
\hline
\end{tabular}


Am. J. Applied Sci., 7 (7): 987-1022, 2010

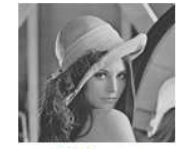

(a)-Lena

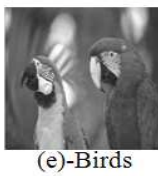

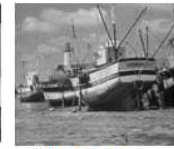

(b)-Boat

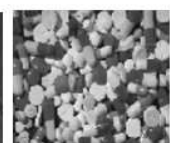

(f)-Pills

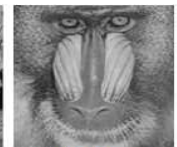

(c)-Baboon

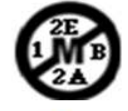

(g)
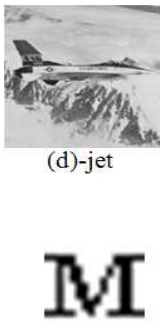

(h)
Fig. 18: (a-f): The watermarked image and ( $g$ and $h)-$ The extracted watermark image

Robustness: We evaluated robustness of the proposed method (APAP-MPOEE-FOBCB ${ }_{\mathrm{MSB}}$ ), under major digital signal processing operations (attacks): Watermark degrading attacks, watermark removal attacks and geometric transformations attacks, by using different benchmark six-test-images (Lena, Boat, Baboon, jet, Birds and Pills) and under using different size of binary watermark image $45 \times 45$ and $16 \times 16$ as shown in Fig. 17, where are used as a tested the performance of capacity, by insertion with redundantly distributed the watermark bits over many pixels of the cover image, by using a small binary watermark image $16 \times 16$, where are added simultaneously in the fallingoff-boundary in corners board pixel to improve the capacity and to ensure robustness. They are good representatives of the more general attacks. Thus will be measure the similarity between the original watermark and extracted watermark, after applying attacks by NCC in Eq. $2 \mathrm{j}$ and SM in Eq. $2 \mathrm{k}$, whereas the similarity values NCC and SM of about 0.75 or above is considered acceptable and with computing psnr between the original watermark and extracted watermark.

The watermark degrading attacks: For adding the Gaussian noise, Salt \& Pepper noise and Speckle noise to the watermarked image, the added noise is a watermark degrading attack, where are add noise can be used as an attacks to remove the watermark. From the performance results are obtained under degrading attacks as shown in the Table 12, comparisons between proposed method (APAP-MPOEE-FOBCB ${ }_{\mathrm{MSB}}$ ) and FOBCB-MSB6 method to study the performance robust results between the constant of the embedding error and proposed method of an adaptively pixel adjustment process based on medial pyramid of embedding error set of the $\mathrm{MSB}_{6}$.

Effect of Gaussian noise: In this experiment we add Gaussian noise to the watermarked image the performance results as shown in Table 10. The extracted watermark image comparator by using NCC, SM and psnr with measuring the PSNR of watermarked image after attacked. The observations of the proposed method are robust under effect of Gaussian noise attacks applying in the different benchmark test images with different size of watermark image inserted. Thus the results are shown in Table 12 shown the PSNR of watermarked image obtained after added Gaussian noise

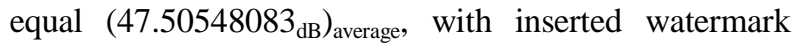
image $45 \times 45$ and equal $\left(47.439372_{\mathrm{dB}}\right)_{\text {average, }}$, with inserted watermark image $16 \times 16$, as seen that by applying proposed method the NCC $=(0.895808)_{\text {average}}, \mathrm{psnr}=$ $\left(8.95452783_{\mathrm{dB}) \text { average }}\right.$ and $\mathrm{SM}=(0.90746467)_{\text {average }}$, with the extracted watermark image $16 \times 16$ and $\mathrm{NCC}=$ $(0.8407545)_{\text {average }}, \mathrm{psnr}=\left(8.25548883_{\mathrm{dB}}\right)_{\text {average }}$ and $\mathrm{SM}$ $=(0.8572943)_{\text {average}}$, with watermark image $45 \times 45$. Results show the proposed method (APAP-MPOEEFOBCB $_{\mathrm{MSB} 6}$ ) deals with Gaussian noise excellently and good robustness. The extracted watermark can maintain a good similarity with the original one even after the watermarked image is adding Gaussian noise and with compared the $\mathrm{FOBCBMSB}_{6}$ method within const-ofembedding error.

Effect of salt and pepper noise: In this experiment we add Salt and Pepper noise to the watermarked image the performance results as shown in Table 12, the PSNR of watermarked image obtained after added Salt and Pepper noise equal (18.4405495 $\mathrm{dB})_{\text {average}}$, with inserted watermark image $45 \times 45$ and equal $\left(18.423221167_{\mathrm{dB}}\right)_{\text {average}}$, with inserted watermark image $16 \times 16$, as seen that by applying proposed method of (APAP-MPOEE-FOBCB ${ }_{\mathrm{MSB}}$ ) the $\mathrm{NCC}=$ $(0.988029167)_{\text {average }}, \mathrm{psnr}=\left(19.48169567_{\mathrm{dB}}\right)_{\text {average }}$ and $\mathrm{SM}=(0.9916935)_{\text {average}}$, with the extracted watermark image $16 \times 16$ and $\mathrm{NCC}=(0.97139767)_{\text {average }}, \mathrm{psnr}=$ $\left(15.7933993_{\mathrm{dB}}\right)_{\text {average }}$ and $\mathrm{SM}=(0.974951)_{\text {average}}$, with watermark image $45 \times 45$. Results show the proposed method (APAP-MPOEE-FOBCB ${ }_{\text {MSB }}$ ) deals with Salt and Pepper noise excellently and good robustness. The extracted watermark can maintain a higher similarity with the original one even after the watermarked image is adding Salt and Pepper noise with heavily degraded of watermarked image and with compared the FOBCBMSB $_{6}$ method within constant of embedding error.

Effect of speckle noise: In this experiment we add Speckle noise to the watermarked image the performance results as shown in Table 13, the PSNR of watermarked image obtained after added Speckle noise equal $\left(47.97788183_{\mathrm{dB}}\right)_{\text {average}}$, with inserted watermark 
Am. J. Applied Sci., 7 (7): 987-1022, 2010

image $45 \times 45$ and equal $\left(47.902916167_{\mathrm{dB}}\right)_{\text {average }}$, with inserted watermark image $16 \times 16$, as seen that by applying proposed method of (APAP-MPOEEFOBCB $\left._{\text {MSB } 6}\right)$ the $\mathrm{NCC}=(0.9049485)_{\text {average }}, \mathrm{psnr}=$ $\left(9.5221683_{\mathrm{dB}}\right)_{\text {average }}$ and SM $(0.91736783)_{\text {average, }}$ with the extracted watermark image $16 \times 16$ and $\mathrm{NCC}=$ $(0.84341883)_{\text {average }}, \mathrm{psnr}=\left(8.4450285_{\mathrm{dB}}\right)_{\text {average }}$ and $\mathrm{SM}$ $=(0.860574167)_{\text {average}}$, with watermark image $45 \times 45$. Results show the proposed method (APAP-MPOEEFOBCB $_{\text {MSB6 }}$ ) deals with Speckle noise excellently and good robustness. The extracted watermark can maintain a high similarity with the original one even after the watermarked image is adding Speckle noise and with compared the $\mathrm{FOBCBMSB}_{6}$ method.

Table 12: Performance results under degrading attacks and comparison

\begin{tabular}{|c|c|c|c|c|c|c|c|c|c|c|c|c|c|c|c|}
\hline \multirow{3}{*}{$\begin{array}{l}\text { Different capacity } \\
\text { of } \mathrm{W}_{(\mathrm{i}, \mathrm{j})} \text { inserted }\end{array}$} & \multirow[b]{3}{*}{ Methods } & \multirow{3}{*}{$\begin{array}{l}\text { Deferent } \\
\text { Bench- } \\
\text { mark test } \\
\text { images }\end{array}$} & \multirow{3}{*}{$\begin{array}{l}\text { Water- } \\
\text { marked } \\
\text { image } \\
\text { PSNR (dB) }\end{array}$} & \multicolumn{12}{|c|}{ The watermark degrading attacks: } \\
\hline & & & & \multicolumn{4}{|c|}{ Gussion noise } & \multicolumn{4}{|c|}{ Salt and paper noise } & \multicolumn{4}{|c|}{ Speckle noise } \\
\hline & & & & PNSR & NCC & psnr & SM & PNSR & $\mathrm{NCC}$ & psnr & SM & PNSR & NCC & psnr & SM \\
\hline \multirow{6}{*}{$\begin{array}{l}\text { Inserted watermark } \\
\text { image size of } 45 \times 45\end{array}$} & \multirow{6}{*}{$\begin{array}{l}\text { The method of } \\
\text { FOBCB-MSB }_{6}\end{array}$} & Lena & 42.29209 & 41.48 & 0.98 & 16.70 & 0.9800 & 18.48 & 0.973 & 15.82 & 0.98 & 41.66 & 0.98 & 17.270 & 0.982 \\
\hline & & Beat & 42.22190 & 41.42 & 0.98 & 17.60 & 0.9840 & 18.57 & 0.968 & 15.28 & 0.97 & 41.59 & 0.98 & 18.010 & 0.985 \\
\hline & & Baboon & 42.03870 & 41.25 & 0.99 & 17.50 & 0.9830 & 18.72 & 0.975 & 16.63 & 0.98 & 41.43 & 0.98 & 17.040 & 0.981 \\
\hline & & Jet & 42.19587 & 41.39 & 0.98 & 16.80 & 0.9810 & 18.03 & 0.974 & 15.43 & 0.97 & 41.09 & 0.98 & 15.990 & 0.976 \\
\hline & & Birds & 42.21755 & 41.41 & 0.99 & 18.90 & 0.9880 & 18.41 & 0.968 & 15.51 & 0.97 & 41.66 & 0.99 & 20.050 & 0.991 \\
\hline & & Pills & 42.10597 & 41.32 & 0.98 & 16.90 & 0.9810 & 18.26 & 0.984 & 16.73 & 0.98 & 41.44 & 0.98 & 18.010 & 0.985 \\
\hline \multirow{6}{*}{$\begin{array}{l}\text { Inserted watermark } \\
\text { image size of } 16 \times 16\end{array}$} & \multirow{6}{*}{$\begin{array}{l}\text { Proposed method } \\
\text { APAP-MPOEE } \\
\text { by FOBCP-MSB } 6\end{array}$} & Lena & 53.29950 & 47.69 & 0.86 & 8.88 & 0.8760 & 18.54 & 0.975 & 16.63 & 0.98 & 48.58 & 0.85 & 8.915 & 0.876 \\
\hline & & Beat & 51.89723 & 47.28 & 0.79 & 8.52 & 0.8600 & 18.59 & 0.968 & 15.28 & 0.97 & 48.06 & 0.75 & 7.737 & 0.830 \\
\hline & & Baboon & 52.48554 & 47.47 & 0.84 & 7.92 & 0.8460 & 18.73 & 0.975 & 16.63 & 0.98 & 48.20 & 0.88 & 8.123 & 0.859 \\
\hline & & Jet & 53.02610 & 47.64 & 0.84 & 7.96 & 0.8490 & 18.05 & 0.974 & 15.43 & 0.97 & 46.49 & 0.81 & 7.131 & 0.816 \\
\hline & & Birds & 52.38855 & 47.44 & 0.84 & 8.37 & 0.8600 & 18.42 & 0.968 & 15.51 & 0.97 & 48.55 & 0.87 & 9.884 & 0.901 \\
\hline & & Pills & 52.54048 & 47.51 & 0.88 & 7.89 & 0.8530 & 18.31 & 0.968 & 15.28 & 0.97 & 47.99 & 0.91 & 8.881 & 0.882 \\
\hline \multirow{6}{*}{$\begin{array}{l}\text { Inserted watermark } \\
\text { image size of } 45 \times 45\end{array}$} & \multirow{6}{*}{$\begin{array}{l}\text { The method of } \\
\text { FOBCB-MSB }_{6}\end{array}$} & Lena & 42.29438 & 41.19 & 1.00 & 24.10 & 0.9970 & 18.52 & 0.994 & 24.08 & 1.00 & 41.37 & 0.99 & 24.080 & 0.997 \\
\hline & & Beat & 41.10731 & 40.47 & 0.99 & 24.10 & 0.9970 & 18.48 & 0.983 & 18.06 & 0.99 & 40.62 & 0.99 & 24.080 & 0.997 \\
\hline & & Baboon & 42.30538 & 41.48 & 0.99 & 24.10 & 0.9970 & 18.72 & 0.983 & 18.06 & 0.99 & 41.66 & 0.99 & 21.070 & 0.994 \\
\hline & & Jet & 41.80323 & 41.08 & 1.00 & 21.10 & 0.9950 & 18.05 & 0.994 & 19.31 & 0.99 & 40.78 & 0.99 & 19.310 & 0.992 \\
\hline & & Birds & 42.19587 & 41.41 & 0.99 & 24.10 & 0.9970 & 18.48 & 0.994 & 19.31 & 0.99 & 41.63 & 0.99 & 21.070 & 0.994 \\
\hline & & Pills & 42.61326 & 41.74 & 0.99 & 21.10 & 0.9940 & 18.21 & 0.983 & 18.06 & 0.99 & 41.86 & 0.99 & 21.070 & 0.994 \\
\hline \multirow{6}{*}{$\begin{array}{l}\text { Inserted watermark } \\
\text { image size of } 16 \times 16\end{array}$} & \multirow{6}{*}{$\begin{array}{l}\text { Proposed method } \\
\text { APAP-MPOEE } \\
\text { by FOBCP-MSB } 6\end{array}$} & Lena & 53.29950 & 47.57 & 0.89 & 9.17 & 0.9100 & 18.59 & 0.989 & 21.07 & 0.99 & 48.46 & 0.94 & 10.470 & 0.936 \\
\hline & & Beat & 51.89723 & 46.80 & 0.82 & 8.06 & 0.8810 & 18.50 & 0.983 & 18.06 & 0.99 & 47.50 & 0.86 & 9.031 & 0.908 \\
\hline & & Baboon & 52.48554 & 47.54 & 0.92 & 9.31 & 0.9180 & 18.70 & 0.989 & 21.07 & 0.99 & 48.31 & 0.92 & 7.955 & 0.890 \\
\hline & & Jet & 53.02610 & 47.64 & 0.90 & 8.90 & 0.9080 & 18.07 & 0.994 & 19.31 & 0.99 & 46.46 & 0.87 & 8.285 & 0.893 \\
\hline & & Birds & 52.38855 & 47.35 & 0.91 & 8.52 & 0.9010 & 18.42 & 0.983 & 18.06 & 0.99 & 48.45 & 0.91 & 11.290 & 0.946 \\
\hline & & Pills & 52.54048 & 47.73 & 0.93 & 9.77 & 0.9260 & 18.26 & 0.989 & 19.31 & 0.99 & 48.24 & 0.93 & 10.100 & 0.931 \\
\hline
\end{tabular}

Table 13: Performance results under geometric attacks and comparison

\begin{tabular}{|c|c|c|c|c|c|c|c|c|c|c|c|c|}
\hline \multirow{3}{*}{$\begin{array}{l}\text { Different capacity } \\
\text { of } W_{(i, j)} \text { inserted }\end{array}$} & \multirow[b]{3}{*}{ Methods } & \multirow{3}{*}{$\begin{array}{l}\text { Deferent } \\
\text { benchmark } \\
\text { test images }\end{array}$} & \multicolumn{10}{|c|}{ Geometric transformations attacks: } \\
\hline & & & \multicolumn{3}{|c|}{ Scaling $(60 \%)$} & \multicolumn{3}{|c|}{ Rotation $30^{\circ} \mathrm{CW}$} & \multicolumn{4}{|c|}{ Geometric distortion } \\
\hline & & & $\mathrm{NCC}$ & psnr & SM & $\mathrm{NCC}$ & psnr & SM & PNSR & $\mathrm{NCC}$ & psnr & SM \\
\hline \multirow{6}{*}{$\begin{array}{l}\text { Inserted watermark } \\
\text { image size of } 45 \times 45\end{array}$} & The method of & Lena & 0.846 & 7.59 & 0.84 & 0.809 & 5.44 & 0.75 & 37.34 & 0.80 & 6.640 & 0.80 \\
\hline & $\mathrm{FOBCB}^{-\mathrm{MSB}_{6}}$ & Beat & 0.846 & 7.59 & 0.84 & 0.781 & 5.57 & 0.75 & 38.94 & 0.78 & 7.477 & 0.82 \\
\hline & & Baboon & 0.846 & 7.59 & 0.84 & 0.816 & 5.50 & 0.76 & 40.18 & 0.81 & 6.791 & 0.80 \\
\hline & & Jet & 0.846 & 7.59 & 0.84 & 0.799 & 5.42 & 0.75 & 40.72 & 0.86 & 6.542 & 0.81 \\
\hline & & Birds & 0.846 & 7.59 & 0.84 & 0.834 & 5.64 & 0.77 & 40.56 & 0.83 & 7.098 & 0.82 \\
\hline & & Pills & 0.846 & 7.59 & 0.84 & 0.845 & 5.51 & 0.77 & 40.28 & 0.80 & 6.842 & 0.81 \\
\hline \multirow{6}{*}{$\begin{array}{l}\text { Inserted watermark } \\
\text { image size of } 16 \times 16\end{array}$} & Proposed method & Lena & 0.846 & 7.59 & 0.84 & 0.801 & 5.33 & 0.75 & 38.70 & 0.55 & 3.868 & 0.59 \\
\hline & APAP-MPOEE & Beat & 0.846 & 7.59 & 0.84 & 0.781 & 5.57 & 0.75 & 40.99 & 0.40 & 3.905 & 0.53 \\
\hline & by FOBCP-MSB 6 & Baboon & 0.846 & 7.59 & 0.84 & 0.816 & 5.50 & 0.76 & 43.57 & 0.59 & 3.624 & 0.59 \\
\hline & & Jet & 0.846 & 7.59 & 0.84 & 0.799 & 5.42 & 0.75 & 44.70 & 0.57 & 3.610 & 0.58 \\
\hline & & Birds & 0.846 & 7.59 & 0.84 & 0.834 & 5.64 & 0.77 & 44.24 & 0.57 & 3.561 & 0.58 \\
\hline & & Pills & 0.846 & 7.59 & 0.84 & 0.845 & 5.51 & 0.77 & 43.79 & 0.67 & 3.595 & 0.62 \\
\hline \multirow{6}{*}{$\begin{array}{l}\text { Inserted watermark } \\
\text { image size of } 45 \times 45\end{array}$} & The method of & Lena & 0.884 & 8.17 & 0.89 & 0.906 & 7.65 & 0.88 & 37.19 & 0.89 & 7.748 & 0.88 \\
\hline & FOBCB-MSB $_{6}$ & Beat & 0.884 & 8.17 & 0.89 & 0.873 & 7.27 & 0.87 & 38.30 & 0.90 & 9.311 & 0.91 \\
\hline & & Baboon & 0.884 & 8.17 & 0.89 & 0.895 & 7.55 & 0.88 & 40.26 & 0.87 & 8.172 & 0.89 \\
\hline & & Jet & 0.884 & 8.17 & 0.89 & 0.906 & 7.27 & 0.87 & 40.33 & 0.91 & 8.519 & 0.90 \\
\hline & & Birds & 0.884 & 8.17 & 0.89 & 0.934 & 7.65 & 0.89 & 40.51 & 0.89 & 9.031 & 0.91 \\
\hline & & Pills & 0.884 & 8.17 & 0.89 & 0.912 & 7.85 & 0.89 & 40.51 & 0.85 & 8.519 & 0.90 \\
\hline \multirow{6}{*}{$\begin{array}{l}\text { Inserted watermark } \\
\text { image size of } 16 \times 16\end{array}$} & Proposed method & Lena & 0.884 & 8.17 & 0.89 & 0.906 & 7.65 & 0.88 & 38.68 & 0.75 & 4.540 & 0.75 \\
\hline & APAP-MPOEE & Beat & 0.884 & 8.17 & 0.89 & 0.873 & 7.27 & 0.87 & 40.86 & 0.75 & 4.260 & 0.75 \\
\hline & by FOBCP-MSB 6 & Baboon & 0.884 & 8.17 & 0.89 & 0.873 & 7.55 & 0.88 & 43.59 & 0.75 & 4.492 & 0.75 \\
\hline & & Jet & 0.884 & 8.17 & 0.89 & 0.876 & 7.55 & 0.87 & 44.71 & 0.75 & 4.215 & 0.75 \\
\hline & & Birds & 0.884 & 8.17 & 0.89 & 0.934 & 7.65 & 0.89 & 44.16 & 0.76 & 4.638 & 0.76 \\
\hline & & Pills & 0.884 & 8.17 & 0.89 & 0.912 & 7.85 & 0.89 & 43.86 & 0.82 & 4.589 & 0.77 \\
\hline
\end{tabular}


Geometric transformations attack: Most attacks will first apply the geometric transformation for e.g., horizontal flip, rotation, cropping, scaling and JPEG compression then save the images with geometric transformation. It makes sense to test robustness of watermarking system to geometric transformation. From the performance results are obtained under the geometric transformations attacks as shown in the Table 13 comparisons between proposed method (APAP-MPOEE-FOBCB ${ }_{\mathrm{MSB}}$ ) and FOBCB-MSB6 $_{-}$ method to study the performance robust results between the constant of the embedding error with the proposed method of an adaptively pixel adjustment process based on medial pyramid of embedding error set of the $\mathrm{MSB}_{6}$.

Re-scaling: The robustness against re-scaling is tested by first resizing the watermarked image to the scaled factor $60 \%$ of its size and then enlarging the image to its original size. Whereas the performance results as shown in Table 13, as seen that by applying proposed method of (APAP-MPOEE-FOBCB $\left.{ }_{\mathrm{MSB} 6}\right)$ the NCC = $(0.883978)_{\text {average }}, \mathrm{psnr}=\left(8.171754_{\mathrm{dB}}\right)_{\text {average }}$ and $\mathrm{SM}=$ $(0.891396)_{\text {average}}$, with the extracted watermark image $16 \times 16$ and $\mathrm{NCC}=(0.846011)_{\text {average}, ~} \mathrm{psnr}=$ $\left(7.586503_{\mathrm{dB}}\right)_{\text {average }}$ and $\mathrm{SM}=(0.837889)_{\text {average}}$, with watermark image $45 \times 45$. The results show the proposed method is resilient in the scaling image of factor $60 \%$, deals to excellently and good robustness. The extracted watermark can maintain a good similarity in both inserted watermark image with the original one even after the watermarked image is re-scaling and with the similarity comparison of the $\mathrm{FOBCBMSB}_{6}$ method.

Re-rotation: In this experiment is tested by first rotate the watermarked image small angle rotation $30^{\circ} \mathrm{CW}$ and then re-rotate the watermarked image to the same angle rotation $30^{\circ} \mathrm{CCW}$ to its original size, the performance results as shown in Table 13, as seen that by applying proposed method of (APAP-MPOEEFOBCB $\left._{\text {MSB6 }}\right)$ the NCC $=(0.89917383)_{\text {average, }}, \mathrm{psnr}=$ $\left(7.58603167_{\mathrm{dB}}\right)_{\text {average }}$ and $\mathrm{SM}=(0.8791805)_{\text {average }}$, with the extracted watermark image $16 \times 16$ and $\mathrm{NCC}=$ $(0.8127705)_{\text {average }}, \mathrm{psnr}=\left(5.492718167_{\mathrm{dB}}\right)_{\text {average }}$ and SM $=(0.75595883)_{\text {average }}$, with watermark image $45 \times 45$. The results show the proposed method is resilient in the rotation image of $30^{\circ} \mathrm{CW}$, deals to excellently and good robustness. The extracted watermark can maintain a good similarity in both inserted watermark image with the original one even after the watermarked image is rerotate and with the comparison of the FOBCBMSB $_{6}$ method.

Geometric distortion with JPEG compression: JPEG called unintentionally attack is currently one of the most widely used compression algorithms for images in order to reduce the file size and save limited bandwidth. In this experiment, the watermarked images are compressed by JPEG standard and the performance results as shown in Table 13, as seen that the PSNR of watermarked image obtained after compressed equal $\left(42.66553183_{\mathrm{dB}}\right)_{\text {average }}$, with inserted watermark image $45 \times 45$ and equal $\left(42.64152167_{\mathrm{dB}}\right)_{\text {average }}$ and with inserted watermark image $16 \times 16$, by proposed method. Furthermore the NCC $=(0.75001633)_{\text {average }}, \mathrm{psnr}=$ $\left(4.4554005_{\mathrm{dB}}\right)_{\text {average }}$ and $\mathrm{SM}=(0.75015367)_{\text {average}}$, of the extracted watermark image $16 \times 16$ and $\mathrm{NCC}=$ $(0.556741)_{\text {average }}, \mathrm{psnr}=(3.693767 \mathrm{~dB})_{\text {average }}$ and $\mathrm{SM}=$ $(0.582182167)_{\text {average }}$ of the extracted watermark image $45 \times 45$. Thus the results shown that the proposed technique deals with geometric distortion of JPEG compression excellently and good robustness only with extracted watermark $16 \times 16$ are inserted with redundantly distributed of the watermark bits over many pixels in the cover image, it is added simultaneously in the falling-off-boundary in corners board pixel to improve the capacity and to ensure robustness. While the $\mathrm{FOBCB}_{\mathrm{MSB} 6}$ method is great a higher robustness with both extracted watermark image. Otherwise the proposed method is robust under extracted watermark $16 \times 16$ and low level with extracted watermark $45 \times 45$.

Horizontal flip: In this experiment we flipped (Horizontal or vertical) the watermarked image without losing any value as well as the proposed scheme is resilience to flipping. Thus the watermark can be well detected and extracted with higher robustness and without any effected distortion.

Cropping: The image cropping is a disposition geometrical attack; in this experiment we crop in the medial of the watermarked image and then the watermark image can be well detected and extracted with high robustness in both methods. Thus the proposed scheme is resilience to cropping.

\section{The watermark removal attacks:}

Changing in lower order bit manipulation of gray values: In this experiment, if the attacker knows that the image has watermark embedded, then the attacker would only have to replace all LSB bits with a ' 1 ' fully defeating the effects or complement the LSB bits and the watermark cannot be recovered from lower order bits $\mathrm{LSB}_{1,2,3,4}$. So that the proposed method (APAPMPOEE-FOBCB ${ }_{\mathrm{MSB} 6}$ ) and the FOBCBMSB $_{6}$ method prevents the attacker to detect or replacing bits, where the PSNR obtained after changing in lower order bit of 
the $\operatorname{LSB}_{1,2,3,4}=31.48 \mathrm{~dB}$ and then can be well detected and extracted watermark with high robustness in both methods.

Altered image: The altered image called a removal attack well here the extraction/detection process for still image is presented. In this experiment we altered image of the watermarked image with other face or other scene. Since that the embedding of watermark bits are in the falling-off-boundary in corners board of cover image. Whereas the watermark image can be well detected and extracted with high robustness in both methods.

Drawing image: The image drawing is a removal attack; in this experiment we drawing on the watermarked image. Thus the watermark image can be well detected and extracted with higher robustness in both methods. Whereas that the embedding of watermark bits are in the falling-off-boundary in corners board of cover image.

\section{CONCLUSION}

The digital watermarking technology is a way to apply digital information hiding techniques to prevent malicious and non-malicious attacks to detect hidden information. Have been proposed a new novel method, fidelity and robust watermark embedding method satisfies the requirements and problems at the same time in an acceptable manner, called adaptively pixel adjustment process based on medial pyramid of embedding error applying in the falling-off-boundary in corners board of the cover image set-of-the MostSignificant-Bit-6 with the random pixel manipulation blind in spatial domain (APAP-MPOEE-FOBCB ${ }_{\mathrm{MSB}}$ ). Furthermore the proposed APAP-MPOEE-FOBCB ${ }_{\text {MSB } 6}$ method using as a embedding watermark bits in the boundary in corners board of the cover image and before embedding requires a checking between the $\mathrm{MSB}_{6}$ in the boundary in corners board pixel of the cover image and EMB of the embedded watermark bit, depending on the nearest of the adaptively pixel in the medial pyramid of embedding error to inform the watermarked image. Experimental results of the proposed method was computed (i) Theoretically are effectiveness in the average of worst case and minimized the embedding error from $\left(2^{\mathrm{n}-1}\right)_{\text {Max }}$ in the maximum pyramid of embedding error to the medial pyramid of embedding error $\left(2^{\mathrm{n}-2}\right)$ medial, where are the embedding error are restricted between the minimum of embedding error ' 1 ' to the medial pyramid of embedding error $\left(2^{\mathrm{n}-2}\right)_{\text {medial }}$, as well as the number of embedding errors $\Omega$ are obtained lowest in the $16_{\text {Max }}$, $8.5_{\text {avrg }}$ and $1_{\text {Min }}$, but within combining between the WMSE and WMSE ${ }^{*}$ reveals that the Max.WMSE ${ }^{*}=\frac{1}{4}$ WMSE are better and in worst case of PSNR $_{\text {worst }}$ equal $\left(24.048_{(\mathrm{dB})}\right)_{\mathrm{Max}}, \quad\left(29.542_{(\mathrm{dB})}\right)_{\text {avg }}$ and $\left(48.130_{(\mathrm{dB})}\right)_{\text {Min }}$ are higher in almost cases of a theoretically computed are proved better than that obtained by the list substitutions of previous methods and modified algorithm. (ii) by the way with applied on the different benchmark of six gray scale images and two quantum of watermark bit embedded are compared with an previous works and modified algorithms was found better. Thus the proposed method leads to imperceptible embed watermark bits with preserving a higher fidelity and good robustness in all different benchmark of test-images the watermarks were extracted from watermark degrading, removal and geometric transformations attacks to an acceptable degree of SM and NCC with extracted watermark image $16 \times 16$ are inserted with redundantly distributed of the watermark bits over many pixels in the cover image, it is added simultaneously in the falling-offboundary in corners board pixel are proved the capacity and ensures robustness, when the watermarks thus embedded were found perceptually non-obstructive on six different gray scale images. Finally, the proposed method, there is no perceptual distortion (fidelity) in the original and watermarked image with PSNR = $\left(52.6062305_{\mathrm{dB}}\right)_{\text {average, }}$ which means that the proposed novel technique has satisfied the criteria that an efficient watermark should be unobtrusive imperceptibility, robust, discreet and easily extracted in spatial domain. By the way from our study of previous methods and modified the algorithms, the our performance results are obtained by computed theoretically and applied on the different benchmark of six gray scale images, we observe that the modified algorithm (PAP-algorithm-3) great a good performance PSNR $=(46.2399895 \mathrm{~dB})_{\text {average }}$ with compared of the previous works and modified algorithms. Further research works may be carried out in spatial domain watermarking to generate: (1): Higher robustness digital watermarking image by applying proposed method with using a factor controller of embedding error. (2): Applying proposed method on the color image to achieve more increasing the capacity and higher robustness.

\section{REFERENCES}

Adil, H. and R. Noumeir, 2008. Methods for image authentication: A survey. Multimedia Tools Appli., 39: 1-46. DOI: 10.1007/s11042-007-0154-3 
Aiad, I. and A. Sada, 2007. Hiding data using LSB-3. J. Basrah Res. Sci., 33: 81-88.

Ali, A.H., 2007. Combined DWT-DCT digital image watermarking. J. Comput. Sci. 3: 740-746.

Arnold, M., S.D. Wolthusen and M. Schmucker, 2003. Techniques and Applications of Digital Watermarking and Content Protection. 1st Edn., Artech House Publishers, London, ISBN: 10: 1580531113, pp: 274.

Bansall, E.A. and S.S. Bhadouria, 2007. Network security and confidentiality with digital watermarking. Proceeding of the Inaugural IEEE International Conference on Digital Ecosystems and Technologies, Feb. 21-23, IEEE Xplore Press, Cairns Australia, pp: 325-328. DOI: 10.1109/DEST.2007.371991

Bijay, S., G.O. Charles and N.H. Younan, 2005. JPEG2000: Image quality metrics. Proceeding of the ASPRS 2005 Annual Conference Geospatial Goes Global: From Your Neighborhood to the Whole Planet, Mar. 7-11, Baltimore, Maryland, pp: $1-11$.

ftp://ftp.ecn.purdue.edu/jshan/proceedings/asprs20 05/Files/0161.pdf

Chan, C.K. and L.M. Cheng, 2004. Hiding data in images by simple LSB substitution. J. Patt. Recog. Soc., $\quad 37$ : 469-474. 10.1016/j.patcog.2003.08.007

Cox, J., M.L. Miller and J.A. Bloom, 2001. Digital Watermarking (The Morgan Kaufmann Series in Multimedia Information and Systems). 1st Edn., Morgan Kaufmann, San Francisco, USA., ISBN: 10: 1558607145, pp: 542.

David, S., G. Motta and D. Bryant, 2007. Data Compression: The Complete Reference. 4th Edn., Springer, USA., ISBN: 10: 1846286026, pp: 1092.

Eric, C., 2003. Hiding in Plain Sight: Steganography and the Art of Covert Communication. 1st Edn., Wiley, Indianapolis, Indiana, ISBN: 10: 0471444499, pp: 360.

Eugene, P.G., 2007. Digital watermarking of bitmap images. Proceedings of the 2007 International Conference on Computer Systems and Technologies, June 14-15, ACM Press, Rousse, Bulgaria, pp: 1-6. DOI: 10.1145/1330598.1330639

Fabien, A.P.P., 2000. Watermarking schemes evaluation. IEEE Sign. Process. Mag. Process., 17: 58-64.

http://www.petitcolas.net/fabien/publications/ieees pm00-evaluation.doc
Fernando, P.G. and J.R. Hernandez, 1999. A tutorial on digital watermarking. Proceeding of the 33rd IEEE Annual Carnahan Conference on Security Technology, Oct. 1999, GPSC, Madrid, Spain, pp: 286-292.

http://www.gts.tsc.uvigo.es/gpsc/publications/wma rk/carnahan99.pdf

Franco, A.D.C. and J.C. Gomez, 2008. DWT based digital watermarking fidelity and robustness evaluation. J. Comput. Sci. Technol., 8: 15-20. http://journal.info.unlp.edu.ar/journal/journal22/pa pers/JCST-Apr08-3.pdf

GraphicsMagick Group, 2002. Compare images: Normalized mean square error. GraphicsMagick Group. http://www.Graphicsmagick.org/api/compare.html

Kamran, H., A. Mumtaz and S.A.M. Gilani, 2006. Digital image watermarking in the wavelet transform domain. Proc. World Acad. Sci., Eng. Technol., 13: 86-89.

Katzenbeisser, S. and F.A.P. Petitcolas, 1999. Information Hiding Techniques for Steganography and Digital Watermarking. 1st Edn., Artech Print, Canton Street Norwood, MA., ISBN: 10: 1580530354, pp: 220.

Kevin, C., X. Li and R. Clarke, 2005. An investigation into the use of the least significant bit substitution technique in digital watermarking. Am. J. Applied Sci., 2: 648-654.

Kutter, M. and F.A.P. Petitcolas, 1999. A fair benchmark for image watermarking systems. Proceeding of the Security and Watermarking of Multimedia Contents, Jan. 25-27, Society of PhotoOptical Instrumentation Engineers, Sans Jose, California, USA., pp: 226-239.

Lesley, R.M., S.G. Mitchell, T.G. Shamoon, R.E. Tarjan and F. Zane, 1998. Robustness and security of digital watermarks. Lecture Notes Comput. Sci., 1465: 227-240.

Lin, L., 2005. A survey of digital watermarking technologies. State University of New York. http://www.ee.sunysb.edu/ cvl/ese558/s2005/Repo rts/Lin\%20Liu/ese558report_LinLiu.pdf

Lu, C.S., 2005. Multimedia Security: Steganography and Digital Watermarking Techniques for Protection of Intellectual Property. 1st Edn., Idea Group Publishing, Taiwan, ROC, ISBN: 10: 1591401925, pp: 350.

Martin, K. and F.A.P. Petitcolas, 2000. Fair evaluation methods for image watermarking systems. J. Elect. Imag., 9: 445-455. DOI: 10.1117/1.1287594 
Matt, L.M., I.J. Cox, M.G. Jean-Paul and L.T. Kalker, 1999. A Review of Watermarking Principles and Practices. In: Digital Signal Processing in Multimedia Systems, Parhi, K.K. and T. Nishitani (Eds.). Marcell Dekker Inc., ISBN: 10: 9783540671824, pp: 461-485.

Mauro, B. and F. Bartolini, 2004. Watermarking Systems Engineering Enabling Digital Assets Security and Other Applications. 1st Edn., CRC Press, New York, ISBN: 10: 0824748069, pp: 500.

MathWorks Inc, 2010. Documentation video and image processing. MathWorks Inc. http://www.mathworks.com/access/helpdesk/ help/toolbox/vipblks/ref/psnr.html

Mehemed, B.A., T.E.A. El-Tobely, M.M. Fahmy, M.E.L. Said Nasr and M.H.A. El-Aziz, 2009. Robust digital watermarking based fallingoffboundary in corners board-MSB-6 gray scale images. Int. J. Comput. Sci. Network Secur., 9: 227-240.

http://paper.ijcsns.org/07_book/html/200908/ 2009 08033.html

Mohanty, S.P., K.R. Ramakrishnan and M. Kankanhalli, 1999. A dual watermarking technique for images. Proceedings of the seventh ACM international Conference on Multimedia (Part 2), Oct. 30-Nov. 5, ACM Press, Orlando, Florida, United States, pp: 49-51. DOI: 10.1145/319878.319891

Satish Kumar, S., 2001. An introduction to image compression. Debugmode. http://www.Debugmode.com/imagecmp/index.htm

Muhammad, S.S. and Y. Dot, 2003. A watermarking scheme for digital images using multilevel wavelet composition. Malaysian J. Comput. Sci., 16: 24-36.

Neil, F.J. and S. Jajodia, 1998. Exploring steganography: Seeing the unseen. IEEE Comput., 31: 26-35. http://www.jjtc.com/pub/r2026.pdf

Sanghyun, J., Y. Suh, J. Shin and H. Kikuchi, 2002. A new robust watermark embedding into wavelet DC components. ETRI J., 24: 401-404. DOI: 10.4218/etrij.02.0202.0502

Santi, P.M. and M.K. Kundu, 2002. Robust and blind spatial watermarking in digital image. Proceedings of the 3rd Indian Conference on Computer Vision, Graphics and Image Processing, Dec. 16-18, Ahmadabad, India, pp: 388-393. http://www.isical.ac.in/ malay/conferen.html
Schyndel, R.G.V., A.Z. Tirke and C.F. Osborne, 1994. A digital watermark. Proceeding of the 1st IEEE Image Processing Conference, Nov. 15-17, RMIT, Houston TX., pp: 86-90. http://goanna.cs.rmit.edu.au/ ronvs/papers/ICIP94. PDF

The Cooke Corporation Kelheim, 2007. SNR-signal-tonoise-ratio. PCO AG. http://www.pco.de/fileadmin/user_upload/db/down load/pco_cooKe_kb_snr_0504.pdf

Tirkel, A.Z., G.A. Rankin, R.M. van Schyndel, W.J. Ho and N.R.A. Mee et al., 1993. Electronic watermark. RMIT.

http://goanna.cs.rmit.edu.au/ ronvs/papers/DICTA 93.PDF

Wang, Y., 2006. Survey of objective video quality measurements. EMC Corporation Hopkinton. ftp://ftp.cs.wpi.edu/pub/techreports/pdf/06-02.pdf

Wang, R.Z., C.F. Lin and J.C. Lin, 2000. Hiding data in images by optimal moderately significant-bit replacement. IEE Elect. Lett., 36: 2069-2070. DOI: IO. 1049/el:20001429

Wikipedia, 2010a. Peak signal-to-noise ratio. Wikipedia. http://en.wikipedia.org /wiki/PSNR.

Wikipedia, 2010b. Signal-to-noise ratio. Wikipedia. http://wapedia.mobi/en/Signal_to_noise_ratio_(ima ge_processing)

Yang, C.H., 2008. Inverted pattern approach to improve image quality of information hiding by LSB substitution. J. Patt. Recog. Soc., 41: 2674-2683. DOI: $10.1016 /$ j.patcog.2008.01.019

Yusnita, Y. and O.O. Khalifa, 2007. Digital watermarking for digital images using wavelet transform. Proceedings of the 2007 IEEE International Conference on Telecommunications and Malaysia International Conference on Communications, May 14-17, IEEE Xplore Press, Penang, Malaysia, pp: 665-669.

Yusnita, Y. and O.O. Khalifa, 2008. Analysis on perceptibility and robustness of digital image watermarking using discrete wavelet transform. Int. J. Comput. Sci. Eng. Syst., 2: 241-244. 